\title{
WestVirginiaUniversity
}

THE RESEARCH REPOSITORY @ WVU

Graduate Theses, Dissertations, and Problem Reports

2012

\section{Magnetic nanoparticles for bio-analytical applications}

Sri Lakshmi Yedlapalli

West Virginia University

Follow this and additional works at: https://researchrepository.wvu.edu/etd

\section{Recommended Citation}

Yedlapalli, Sri Lakshmi, "Magnetic nanoparticles for bio-analytical applications" (2012). Graduate Theses, Dissertations, and Problem Reports. 4942.

https://researchrepository.wvu.edu/etd/4942

This Dissertation is protected by copyright and/or related rights. It has been brought to you by the The Research Repository @ WVU with permission from the rights-holder(s). You are free to use this Dissertation in any way that is permitted by the copyright and related rights legislation that applies to your use. For other uses you must obtain permission from the rights-holder(s) directly, unless additional rights are indicated by a Creative Commons license in the record and/ or on the work itself. This Dissertation has been accepted for inclusion in WVU Graduate Theses, Dissertations, and Problem Reports collection by an authorized administrator of The Research Repository @ WVU.

For more information, please contact researchrepository@mail.wvu.edu. 
MAGNETIC NANOPARTICLES FOR BIO-ANALYTICAL APPLICATIONS

By

Sri Lakshmi Yedlapalli

Dissertation submitted to the Eberly College of Arts and Sciences at West Virginia University

in partial fulfillment of the requirements

for the degree of

Doctor of Philosophy

in

Chemistry

\author{
Approved by \\ Lisa A. Holland, Ph.D., Committee Chairperson \\ Letha J. Sooter, Ph.D., Advisor \\ Harry O. Finklea, Ph.D. \\ Jonathan Boyd, Ph.D. \\ Justin Legleiter, Ph.D.
}

C. Eugene Bennett Department of Chemistry

Morgantown, West Virginia

2012

Keywords: SPIONs, Drug delivery, Aptamers, Binding Affinity, Capillary Electrophoresis 


\section{ABSTRACT \\ Magnetic nanoparticles for Bio-analytical Applications \\ Sri Lakshmi Yedlapalli}

Magnetic nanoparticles are widely being used in various fields of medicine, biology and separations. This dissertation focuses on the synthesis and use of magnetic nanoparticles for targeted drug delivery and analytical separations. The goals of this research include synthesis of biocompatible surface modified monodisperse superparamagnetic iron oxide nanoparticles (SPIONs) by novel techniques for targeted drug delivery and use of SPIONs as analytical sensing tools. Surface modification of SPIONs was performed with two different copolymers: tri block copolymer Pluronics and octylamine modified polyacrylic acid.

Samples of SPIONs were subsequently modified with 4 different commercially available, FDA approved tri-block copolymers (Pluronics), covering a wide range of molecular weights $(5.75-14.6 \mathrm{kDa})$. A novel, technically simpler and faster phase transfer approach was developed to surface modify the SPIONs with Pluronics for drug delivery and other biomedical applications. The hydrodynamic diameter and aggregation properties of the Pluronic modified SPIONs were studied by dynamic light scattering (DLS). The coverage of SPIONs with Pluronics was supported with IR Spectroscopy and characterized by Thermo gravimetric Analysis (TGA). The drug entrapment capacity of SPIONs was studied by UV-VIS spectroscopy using a hydrophobic carbocyanine dye, which serves as a model for hydrophobic drugs. These studies resulted in a comparison of physical properties and their implications for drug loading capacities of the four types of Pluronic coated SPIONs for drug delivery assessment. These drug delivery systems could be used for passive drug targeting. However, Pluronics lack the functional group necessary for bioconjugation and hence cannot achieve active targeting. 
SPIONs were functionalized with octylamine modified polyacrylic acid-based copolymer, providing water solubility and facile biomolecular conjugation. Epirubicin was loaded onto SPIONs and the drug entrapment was studied by UVVIS spectrophotometry. In this study, the antisense oligonucleotide sequence to the anti-apoptopic protein survivin was coupled to SPIONs to provide molecular targeting and potential therapy for cancer cells. Successful coupling of antisense survivin to SPIONs was demonstrated by circular dichroism studies of the conjugate and its complementary sequence. Such multifunctional SPIONs can be used as active targeting agents for cancer cells, producing enhanced magnetic resonance imaging contrast and releasing chemotherapeutic agents to targeted cells.

SPIONs also serve as an excellent platform for analytical sensing. Streptavidin modified SPIONs were used as substrates to immobilize biotinylated aptamers (single-stranded DNA). The binding affinity of such aptamers to its target was achieved by quantifying the amount of target released from the aptamer. This quantification was achieved using $\mathrm{pH}$-mediated stacking capillary electrophoresis. SPIONs were shown to be more efficient compared to magnetic microbeads as the sensing elements. The binding affinity constant of the aptamer determined was almost 8-fold better than that obtained using magnetic microbeads. 


\section{Dedication}

I am grateful to my parents, Lakshmi Durga and Chandra Sekhar Rao Yedlapalli who set foundation for my best education and always believed in me. I would like to express my sincere love to my sister, Sri Devi Yedlapalli who always encouraged me.

I express my heartfelt thanks to my husband Chandrakanth Apala for his constant motivation and encouragement in completing my research work and in writing my dissertation. He was always there for me giving his moral support during my entire graduate life. It is his outmost love and care for me that made me successful.

This work is dedicated to all of these people who love and care for me and believed in what I am and who I can be. 


\section{Acknowledgements}

First, I would like to thank Dr. Letha Sooter who supported me in completing my research work. I would like to express my sincere thanks to Dr. Lisa Holland for her patient guidance; tremendous motivation and encouragement which helped me complete my dissertation. I learnt how to think critically and attained a complete professional training to proceed further in my career under both of their guidance.

I would like to thank Dr. R. Lloyd Carroll for his guidance and encouragement. I would like to thank all of my doctoral committee members: Dr. Lisa Holland, Dr. Letha Sooter, Dr. Harry Finklea, Dr. Jonathan Boyd and Dr. Justin Legleiter for their support, instruction and time. Thanks to Dr. Peter Gannett for the collaboration work with SPIONs, Dr. Mohindar Seehra for his help with XRD, Dr. Marcela Redigalo for her help with TEM.

I would like to thank C.Eugene Bennett Department of Chemistry for accepting me to the graduate program and providing financial assistance. My sincere thanks to all the faculty members for the invaluable knowledge and experience I gained from the courses, seminars and teaching.

I would like to thank Eberly college of Arts \& Sciences for travel grants which made it possible for me to present my work at national meetings. I thank WVU Graduate life \& education for giving me Dissertation fellowship which helped me to focus on my writing and finish it on time.

My special thanks to Aaron, Garrett, Sunny and Vincent who worked with me on this research work. Finally I would like to thank all my labmates whom I worked with: Shengrong, Ichuk, Ryan, Amanda, Kailey, Nick, Ted, Tyler, Anthony, Brandon and Xingwei for their time, support and friendship. Thanks to my family and friends for their support. 


\section{Table of Contents}

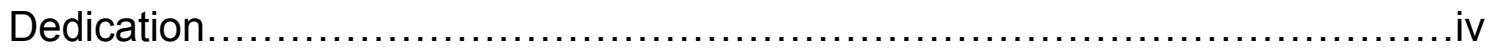

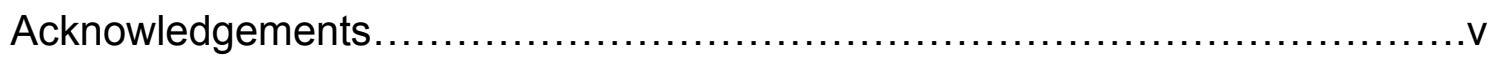

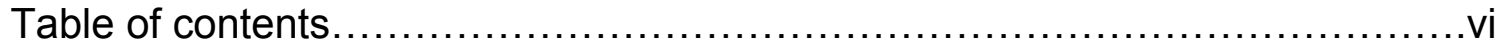

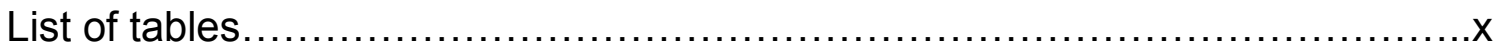

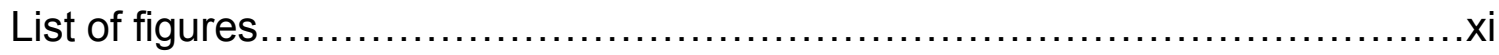

List of symbols and nomenclature ..............................................

Chapter 1: Introduction.............................................................

1.1 Synthesis of magnetic iron oxide nanoparticles............................. 4

1.2 Surface modifications on magnetic nanoparticles...........................6

1.3 Bio-analytical applications of magnetic nanoparticles.......................6

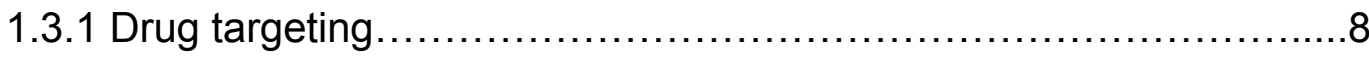

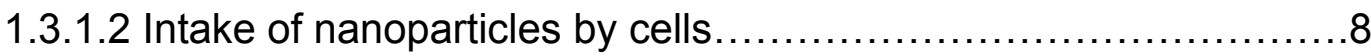

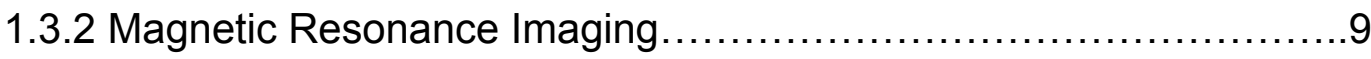

1.3.3 Hyperthermia..................................................... 10

1.3.4 Biological molecule separations .................................10

1.4 Objectives of my research....................................................

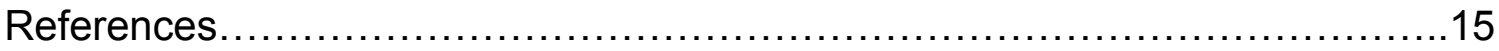

Chapter 2: Pluronic triblock copolymer-modified mono disperse

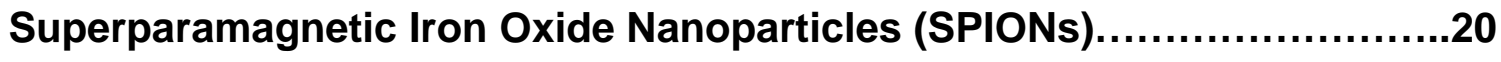

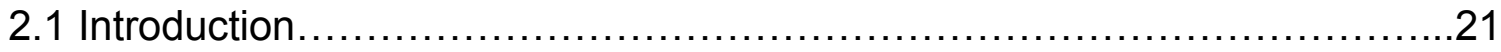

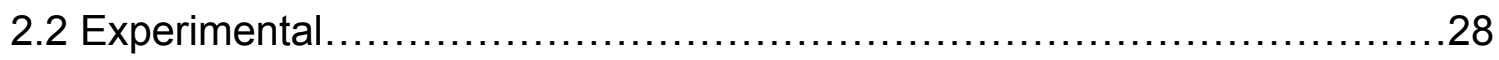

2.2.1 Materials and Methods ............................................. 
2.2.1.1 Reagents and materials.

2.2.1.2 Synthesis of monodisperse magnetic nanoparticles by thermal

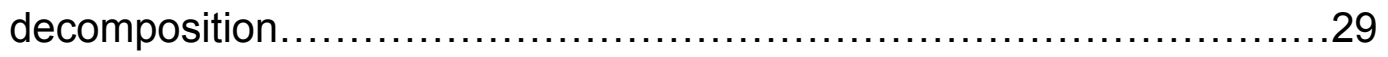

2.2.1.3 Preparation of Pluronic coated OA-SPIONs......................29

2.2.1.4 Loading of Dil dye onto Pluronic coated SPIONs..................30

2.2.2 Characterization .................................................

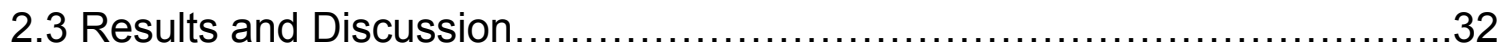

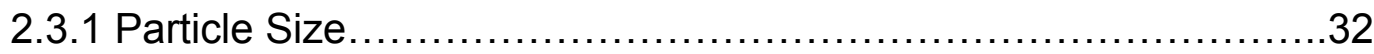

2.3.2 Pluronic adsorbed on SPIONs....................................

2.3.3 Loading of hydrophobic dye Dil onto Pluronic coated SPIONs.......43

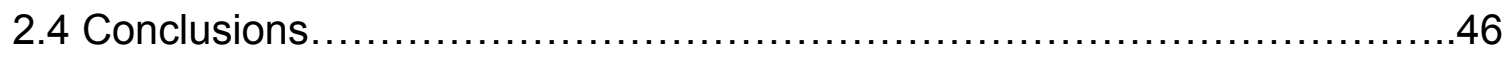

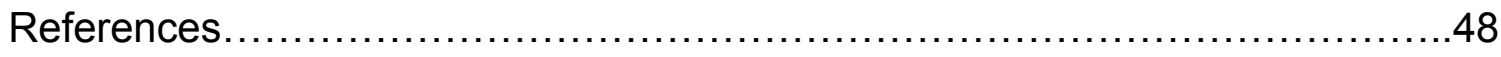

Chapter 3: Antisense Survivin coupled surface modified magnetic

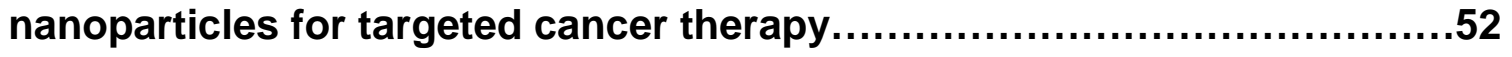

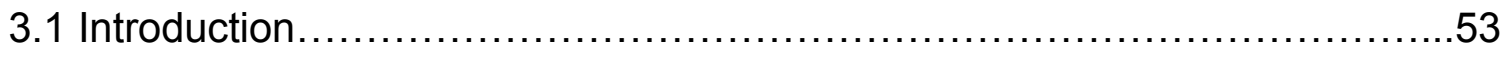

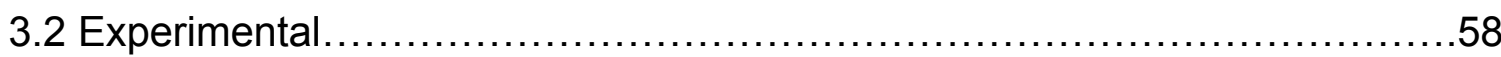

3.2.1 Materials and methods .......................................... 58

3.2.2 Synthesis and characterization of OA-SPIONs .....................59

3.2.3 Surface modification of SPIONs by modified polyacrylic acid........59

3.2.4 Oligonucleotide Synthesis and purification.........................60

3.2.5 Coupling of antisense Survivin to SPIONs........................61

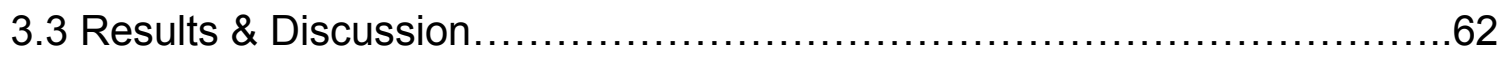

3.3.1 Fourier Transform Infrared Spectroscopy ........................62 
3.3.2 Dynamic Light Scattering of PAA-OA-SPIONs......................65

3.3.3 Loading of Epirubicin onto PAA-OA-SPIONs.......................65

3.3.4 Coupling of fluorescent antisense surviving ligand (f-aSL) to

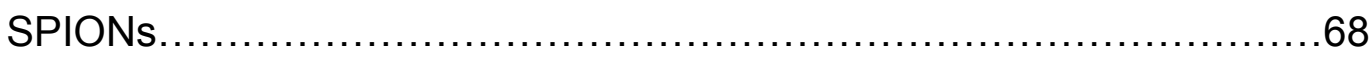

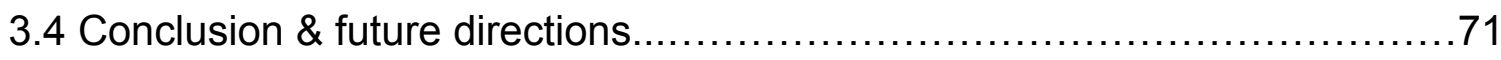

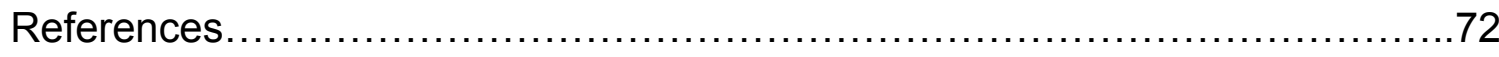

Chapter 4: Superparamagnetic Iron Oxide Nanoparticles (SPIONs) as a

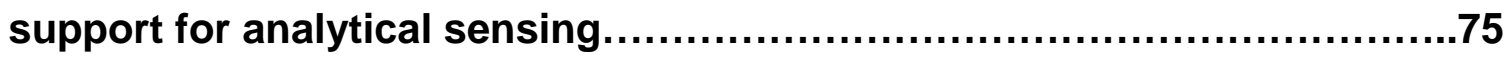

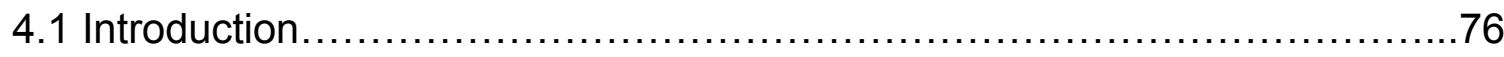

4.1.1Theory of Capillary electrophoresis................................ 79

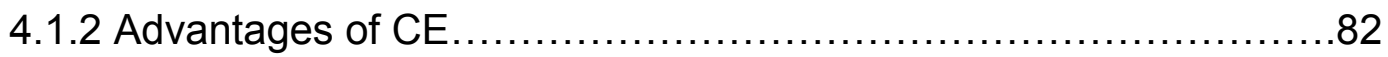

4.1.3 Micellar electrokinetic chromatography (MEKC) ...................83

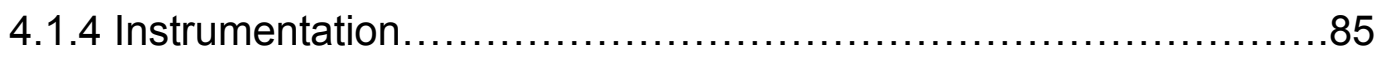

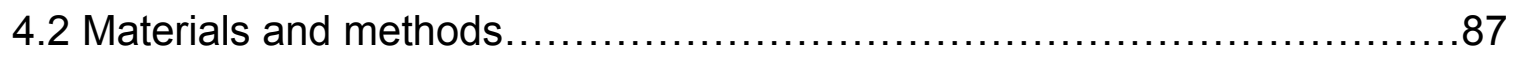

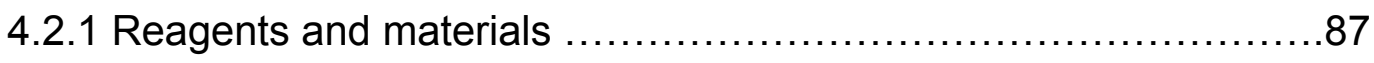

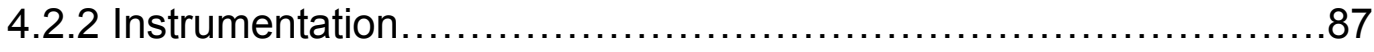

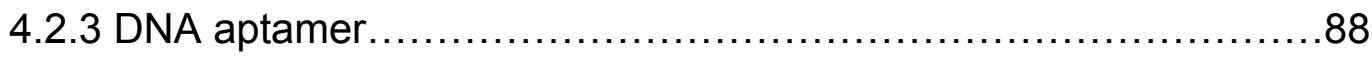

4.2.4 Capillary Electrophoresis separation conditions....................88

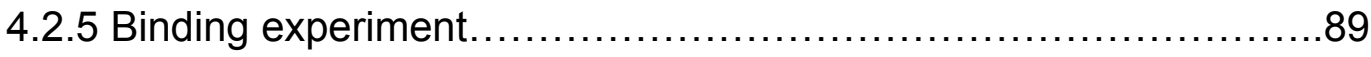

4.2.6 Separation mechanism........................................ 90

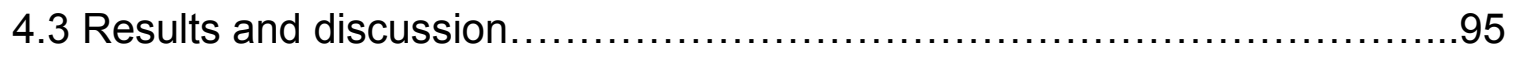

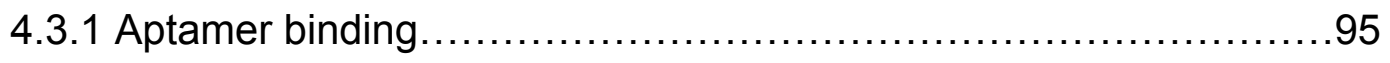

4.3.2 Standard addition method .......................................95 


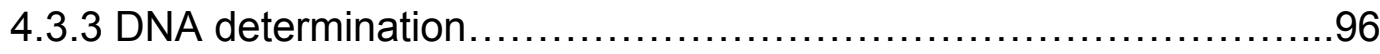

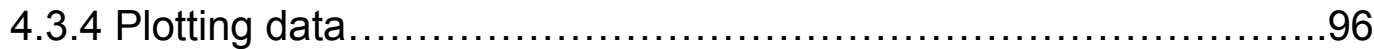

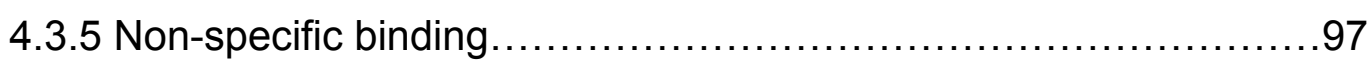

4.3.6 "Magnetic micro particles" Vs "magnetic nanoparticles" .............97

4.4 Conclusions and future directions ......................................... 103

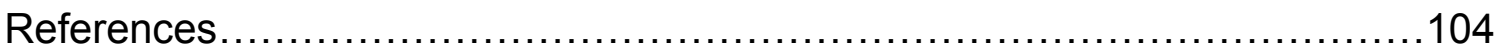




\section{List of tables}

Table 1-1: Summary comparison of the synthetic methods.....................5

Table 2-1: Summary of Pluronic polymers studied in this work................24

Table 2-3: Analysis of TGA results..........................................

Table 3-1: Data obtained from fluorescence spectroscopy showing the

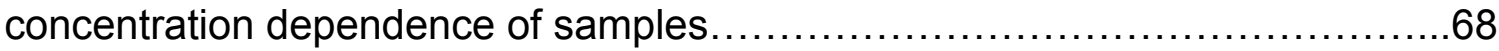

Table 4-1: Comparison of $\mathrm{Kd}$ and Bmax values obtained from the aptamer

binding studies done using magnetic micro and nano particles.................99

Table 4-2: Raw Data Used for each Binding Isotherm..........................102 


\section{List of figures}

Figure 1-1: Accumulation of nanoparticles by EPR effect [Passive targeting] ......3

Figure 1-2: Applications of magnetic nanoparticles.............................

Figure 1-3: Schematic of online preconcentration and selective separation of lipoproteins using multifunctional magnetic particles (MFMPs) in affinity CE....13

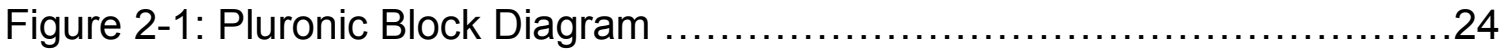

Figure 2-2: Structure of 1,1'-dioctadecyl-3,3,3'3'-tetramethylindocarbocyanine per

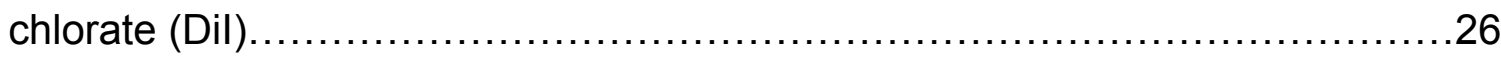

Figure 2-3: Schematic representation of Dil dye loaded Pluronic-OA-SPIONs

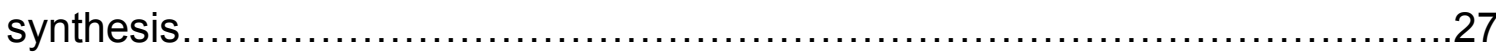

Figure 2-4: Pluronic SPIONs in aqueous dispersion...........................30

Figure 2-5.a: TEM image of OA-SPIONs in toluene; inset shows HRTEM image

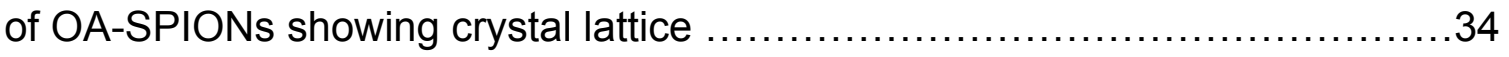

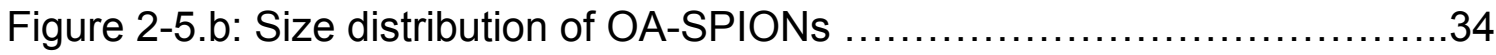

Figure 2-5.c: TEM image of Pluronic-OA-SPIONs..............................34

Figure 2-6: XRD patterns of oleic acid coated SPIONs .........................36

Figure 2-7: FTIR spectra of OA-SPIONs, Pluronic-OA-SPIONs and free pluronic F127 as reference showing the functional groups............................ 38

Figure 2-8. a: Graphical Representation of hydrodynamic size variation of

Pluronic coated OA-SPIONs as synthesized.

Figure 2-8.b: Graphical Representation of hydrodynamic size variation of

Pluronic coated OA-SPIONs after 6 months. 
Figure 2-9: TGA of SPIONs and Pluronic coated SPIONs showing the degradation of organic polymer at high temperatures

Figure 2-10. a: UV-Visible absorbance of OA-Pluronic SPIONs with entrapped Dil dye .45

Figure 2-10. b: Comparison of hydrophobic dye entrapment efficiency of OA-

Pluronic SPIONs. .45

Figure 3-1: Chemical structure of Epirubicin. 56

Figure 3-2: Schematic of synthesis of Antisense Survivin DNA coupled magnetic nanoparticles. 57

Figure 3-3: FTIR spectra of SPIONs and modified PAA coated SPIONs 64

Figure 3-4. UV-Visible Spectroscopy of Epirubicin. 67

Figure 3-5: Emission spectra of f-aSL and f-aSL:SPIONs at various concentrations 69

Figure 4-1: Chemical structure of $17 \beta$-estradiol (E2). 78

Figure 4-2: Charged molecule placed in a constant electric field experiencing different types of forces. .79

Figure 4-3: Block diagram of capillary electrophoresis.........................80

Figure 4-4: Representation of Electro-osmotic flow............................ 81

Figure 4-5: Representation of Electrophoretic mobility ........................82

Figure 4-6: Structures of sodium dodecyl sulfate and cyclodextrin...............84

Figure 4-7: Photographs of the Beckman® automated capillary electrophoresis machine used in the work. .86

Figure 4-8: Denaturing of DNA aptamer and its renaturing at room temp. 92 
Figure 4-9: Schematic of SPIONs washing procedure ........................92

Figure 4-10: Schematic of binding affinity study of 76 mer with estradiol using

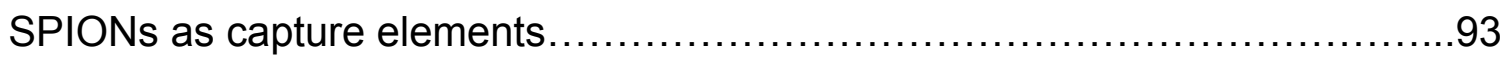

Figure 4-11: Schematic of $\mathrm{pH}$ mediated micellar electrokinetic chromatography

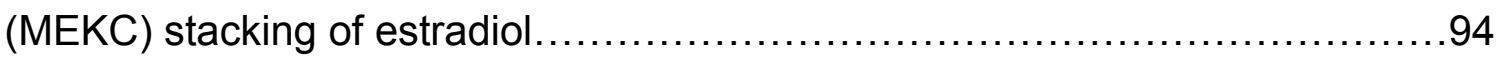

Figure 4-12: Representative electropherogram showing a single standard

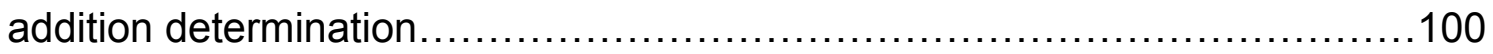

Figure 4-13: Represents three determinations of binding affinity of $76 \mathrm{mer}$ aptamer and steroids using SPIONs as capture elements.....................101 


\section{List of Symbols and Nomenclature}

${ }^{\circ} \mathrm{C}$ - degrees Celsius

A - Adenine

ACE - affinity capillary electrophoresis

aSL - antisense surviving ligand

BGE - background electrolyte

$\mathrm{B}_{\max }$ - maximum binding of receptor

C - cytosine

CAPS - 3-[cyclohexylamino]-1-propanesulfonic acid

CE - capillary electrophoresis

$\mathrm{cm}$ - centimeter; 1 x 10-2 m

CD - cyclodextrin

CMC - critical micellar concentration

CMCD - carboxymethyl- $\beta$-cyclodextrin

CZE - capillary zone electrophoresis

$\mathrm{Da}$ - dalton

Dil - 1,1'-dioctadecyl-3,3,3'3'-tetramethylindocarbocyanine per chlorate

DLS - dynamic light scattering

DMF - dimethyl formamide

DNA - deoxyribonucleic acid

E2 - 17b-estradiol

EDC - 1-Ethyl-3-(3-dimethylaminopropyl)carbodiimide

EOF - electroosmotic flow 
EPI - epirubicin

$f-$ frictional coefficient

f-aSL - fluorescent antisense surviving ligand

G - guanine

FTIR - Fourier transform infra red spectroscopy

h - hour

$\mathrm{HCl}$ - hydrochloric acid

HLB - hydrophilic lipophilic balance

HPCD - hydroxypropyl- $\beta$-cyclodextrin

HV - high voltage

i.d. - inner diameter

$\mathrm{k}$ - rate constant

$\mathrm{K}_{\mathrm{d}}$ - dissociation constant

$\mathrm{kDa}$ - kilo daltons

kV - kilovolts; 1 x $103 \mathrm{~V}$

L - ligand

LOD - limit of detection

M - molar

MEKC - micellar electrokinetic chromatography

MFMP - multifunctional magnetic particles

MRI - magnetic resonance imaging

mg - milligram; 1 x 10-3 g

min - minutes 


$$
\begin{aligned}
& \mathrm{mL} \text { - milliliter; } 1 \times 10-3 \mathrm{~L} \\
& \mathrm{mM} \text { - millimolar; } 1 \times 10-3 \mathrm{M} \\
& \mu \mathrm{A} \text { - microamp; } 1 \times 10-6 \mathrm{~A} \\
& \mu_{\mathrm{ap}} \text { - apparent mobility } \\
& \mu_{\mathrm{ep}} \text { - electrophoretic mobility } \\
& \mu_{\text {eof }} \text { - electroosmotic mobility } \\
& \mu \mathrm{g} \text { - microgram; } 1 \times 10-6 \mathrm{~g} \\
& \mu \mathrm{L} \text { - microliter; } 1 \times 10-6 \mathrm{~L} \\
& \mu \mathrm{m} \text { - micrometer; } 1 \times 10-6 \mathrm{~m} \\
& \mu \mathrm{M} \text { - micromolar; } 1 \times 10-6 \mathrm{M} \\
& \mathrm{nL}-\text { nanoliter; } 1 \times 10-9 \mathrm{~L} \\
& \mathrm{~nm} \text { - nanometer; } 1 \times 10-9 \mathrm{~m} \\
& \mathrm{NaOH} \text { - sodium hydroxide } \\
& \mathrm{NS} \text { - slope of nonspecific binding plot } \\
& \mathrm{NSB} \text { - nonspecific binding } \\
& \mathrm{OA} \text { - oleic acid } \\
& \mathrm{PAA} \text { - polyacrylic acid } \\
& \mathrm{PCR} \text { - polymerase chain reaction } \\
& \mathrm{PDA} \text { - photodiode array } \\
& \mathrm{PEO} \text { - polyethylene oxide } \\
& \mathrm{PPO} \text { - polypropylene oxide } \\
& \mathrm{psi}
\end{aligned}
$$


$r$ - hydrodynamic radius

RNA - ribonucleic acid

RPM - rotations per minute

RS - resolution

RSD - relative standard deviation

$\mathrm{S} / \mathrm{N}$ - signal to noise

SDS - sodium dodecyl sulfate

sec -seconds

SELEX - Systematic Evolution of Ligands by Exponential Enrichment

SPIONs - Superparamagnetic Iron oxide nanoparticles

$\mathrm{T}$ - thymine

TEM - Transmission electron microscope

TGA - Thermogravimetric analysis

$\eta-$ Viscosity

XRD - X-ray diffraction 
Chapter 1: Introduction 
Nanoparticles, due to their size, offer a number of special optical ${ }^{1}$, electronic ${ }^{2}$ properties. The physico-chemical properties of nanoparticles differ vastly depending on their dimensions, charge, hydrophobicity and the surface coating. Magnetic nanoparticles have an additional property of responding to magnetic field. ${ }^{3}$

Magnetic particles ranging from nanometer $(\mathrm{nm})$ to micrometer (um) size are widely being used in the various fields such as sensing, diagnostics and drug delivery. ${ }^{4,5}$ Successful application of the nanoparticles in these areas is highly dependent on the stability of these particles under different aqueous and physiological conditions. The ability to solubilize the nanoparticles in water and to modify their surface with molecules, proteins or other targeting agents is a crucial step for their biomedical applications and bioanalysis. ${ }^{6}$

In the last two decades, the use of magnetic nanoparticles in biomedical applications has been increasing, especially in the treatment of cancer through tumor targeting. Though there are different ways to treat cancer, such as surgery, radiation, chemotherapy etc., the most widely used approach is chemotherapy, but there are a number of limitations with conventional chemotherapeutic approaches. The major disadvantage in conventional chemotherapeutic approaches for cancer treatment is non-specificity that results in undesirable side effects. $^{7}$ The use of nanoparticles as drug delivery vectors can revolutionize 
cancer therapy. Magnetic nanoparticles can be used to target specific site of cancer. As nanoparticles are very small, they are easily accepted by cells, and hence many small molecules, nucleic acids, peptides are being loaded into nanoparticles and targeted to the specific tissues. SPIONs are FDA approved and are being used in clinical applications. ${ }^{8}$

Using nanoparticles in drug delivery reduces the drug dose and improves drug efficiency. Since most normal tissues have non-leaky microvasculature, long circulating nanoparticles accumulate to a greater extent in the leaky tumor tissue with poor lymphatic drainage system. This passive targeting of the drug is achieved by enhanced permeability and retention effect. Prolonged circulation of the drug results in better targeting efficiency due to greater accumulation of drug particles in the tumor tissue. ${ }^{9}$

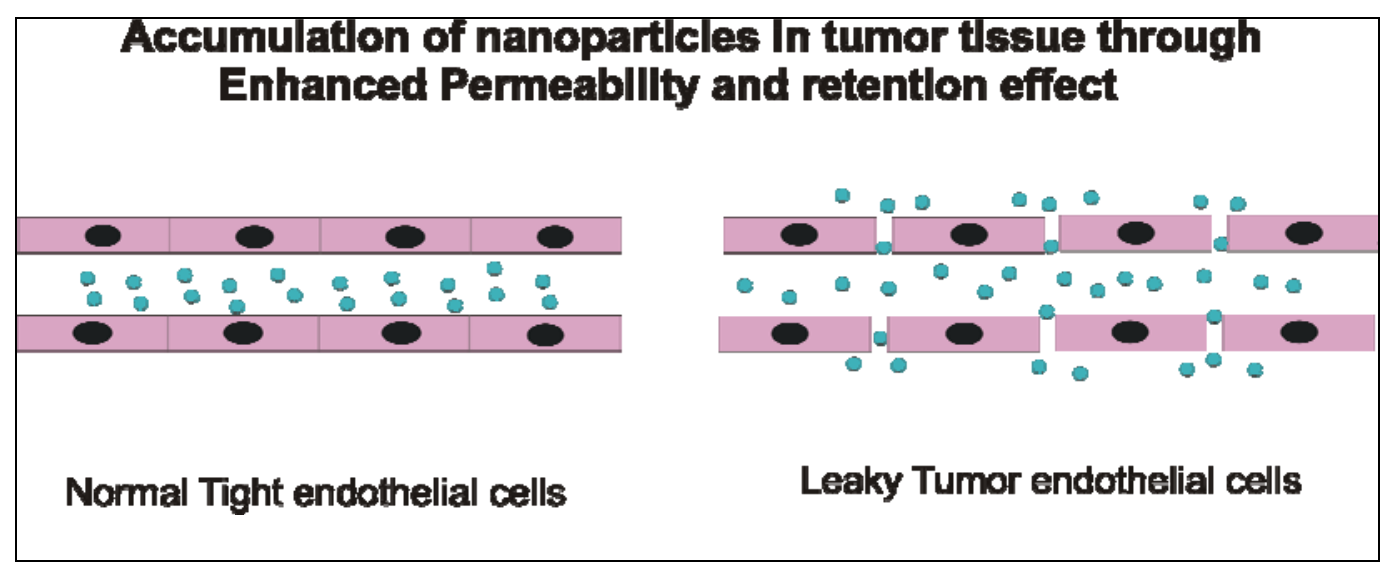

Figure 1-1: Accumulation of nanoparticles by Enhanced permeability and retention effect [Passive targeting] 
Iron Oxide is the most commonly used metal in nanoparticle formulations. Two types of Iron oxides have been investigated for use in magnetic nanoparticle formulations: Maghemite $\left[\mathrm{\gamma}-\mathrm{Fe}_{2} \mathrm{O}_{3}\right]$ and Magnetite $\left[\mathrm{Fe}_{3} \mathrm{O}_{4}\right]$. Magnetic nanoparticles are potentially used in biomedical applications due to their high stability and non-toxic nature. ${ }^{6}$

Depending on the size of the magnetic nanoparticles, they are named as superparamagnetic iron oxide nanoparticles (SPIONs). A superparamagnetic material is composed of small ferromagnetic crystallites which randomly flip their magnetic moment direction under thermal fluctuations. As a result, the material as a whole is not magnetized except in an externally applied magnetic field (similar to paramagnetic material). Due to this magnetic nature, SPIONs can be used as site specific release drug carriers, magnetic resonance imaging (MRI) agents and in hyperthermia. SPIONs with core sizes ranging from 3 to $10 \mathrm{~nm}$ with dextran polymer coating are approved for use as MRI contrast agents in patients. Commercially available iron oxide formulations for MRI include Ferridex, Combidex and Sinerem. ${ }^{10}$

\subsection{Synthesis of magnetic iron oxide nanoparticles}

Iron Oxide Nanoparticles were produced by many different methods. These include co-precipitation, thermal decomposition, microemulsion and hydrothermal synthesis ${ }^{11}$. However, thermal decomposition is preferred in terms of size and 
morphology control of nanoparticles. ${ }^{12}$ Table-1 shows the summary comparison of the synthetic methods.

Table 1-1: Summary comparison of the synthetic methods.

\begin{tabular}{|c|c|c|c|c|c|}
\hline $\begin{array}{l}\text { Synthetic } \\
\text { method }\end{array}$ & Synthesis & Solvent & $\begin{array}{c}\text { Size } \\
\text { Distribution }\end{array}$ & $\begin{array}{l}\text { Shape } \\
\text { control }\end{array}$ & Yield \\
\hline Co-precipitation & $\begin{array}{l}\text { Very simple, } \\
\text { ambient } \\
\text { conditions }\end{array}$ & Water & $\begin{array}{c}\text { Relatively } \\
\text { narrow }\end{array}$ & $\begin{array}{l}\text { Not } \\
\text { good }\end{array}$ & $\begin{array}{c}\text { High/ } \\
\text { scalable }\end{array}$ \\
\hline $\begin{array}{c}\text { Thermal } \\
\text { decomposition }\end{array}$ & $\begin{array}{c}\text { Complicated, } \\
\text { inert } \\
\text { atmosphere }\end{array}$ & $\begin{array}{c}\text { Organic } \\
\text { compound }\end{array}$ & Very narrow & $\begin{array}{l}\text { Very } \\
\text { good }\end{array}$ & $\begin{array}{c}\text { High/ } \\
\text { Scalable }\end{array}$ \\
\hline microemulsion & $\begin{array}{l}\text { Complicated, } \\
\text { ambient } \\
\text { conditions }\end{array}$ & $\begin{array}{l}\text { Organic } \\
\text { compound }\end{array}$ & $\begin{array}{c}\text { Relatively } \\
\text { narrow }\end{array}$ & Good & Low \\
\hline $\begin{array}{c}\text { Hydrothermal } \\
\text { synthesis }\end{array}$ & $\begin{array}{l}\text { Simple, high } \\
\text { pressure }\end{array}$ & $\begin{array}{l}\text { Water } \\
\text { ethanol }\end{array}$ & Very narrow & $\begin{array}{l}\text { Very } \\
\text { good }\end{array}$ & medium \\
\hline
\end{tabular}

For all these methods a surface capping agent is needed and added during the synthesis. 


\subsection{Surface modifications on magnetic nanoparticles}

Size and surface characteristics are two important parameters which highly dictate particle stability and interactions of the particles with biological environment. To make these nanoparticles biocompatible and attach biomolecules, they must undergo surface modifications. ${ }^{4}$ 13 Magnetic nanoparticles have been surface modified with different polymers such as polyethylene glycol (PEG), polyacrylic acid (PAA), polyvinyl alcohol (PVA), dextran etc. Alteration of the surface chemistry and physical properties enables in preparation of functional magnetic nanoparticles apart from providing increased stability. Stabilization of nanoparticles by surfactants or polymers can be due to electrostatic repulsion of surface charges or steric stabilization. Polymers can attach to nanoparticles by physical adsorption due to weak Vander Waals forces, dipole-dipole interactions or hydrogen bonds and chemical adsorption by electrostatic or covalent bonds. Surface modified nanoparticles also help in coupling of drugs by covalent attachment, adsorption or entrapment. Target biomolecules such as antibodies and nucleic acids can also be conjugated to functionalized nanoparticles. Thus, surface engineering of nanoparticles results in their applications in various fields.

\subsection{Bio-analytical applications of magnetic nanoparticles}

In the last two decades, magnetic nanoparticles are extensively being used in number of bioanalytical applications. ${ }^{4,14,15}$ The use of magnetic nanoparticles can be classified based on their in vivo and in vitro applications. In vivo 
applications of magnetic nanoparticles includes their effective use as contrast agents in magnetic resonance imaging (MRI), selective destruction of malignant tissues through heating by a technique called hyperthermia and as site selective delivery vehicles for drugs. In vitro applications of magnetic nanoparticles include their use as separation tools in nano and microfluidic devices as well as biological or immunoassays. Figure 1-2 shows some of the applications of magnetic nanoparticles.

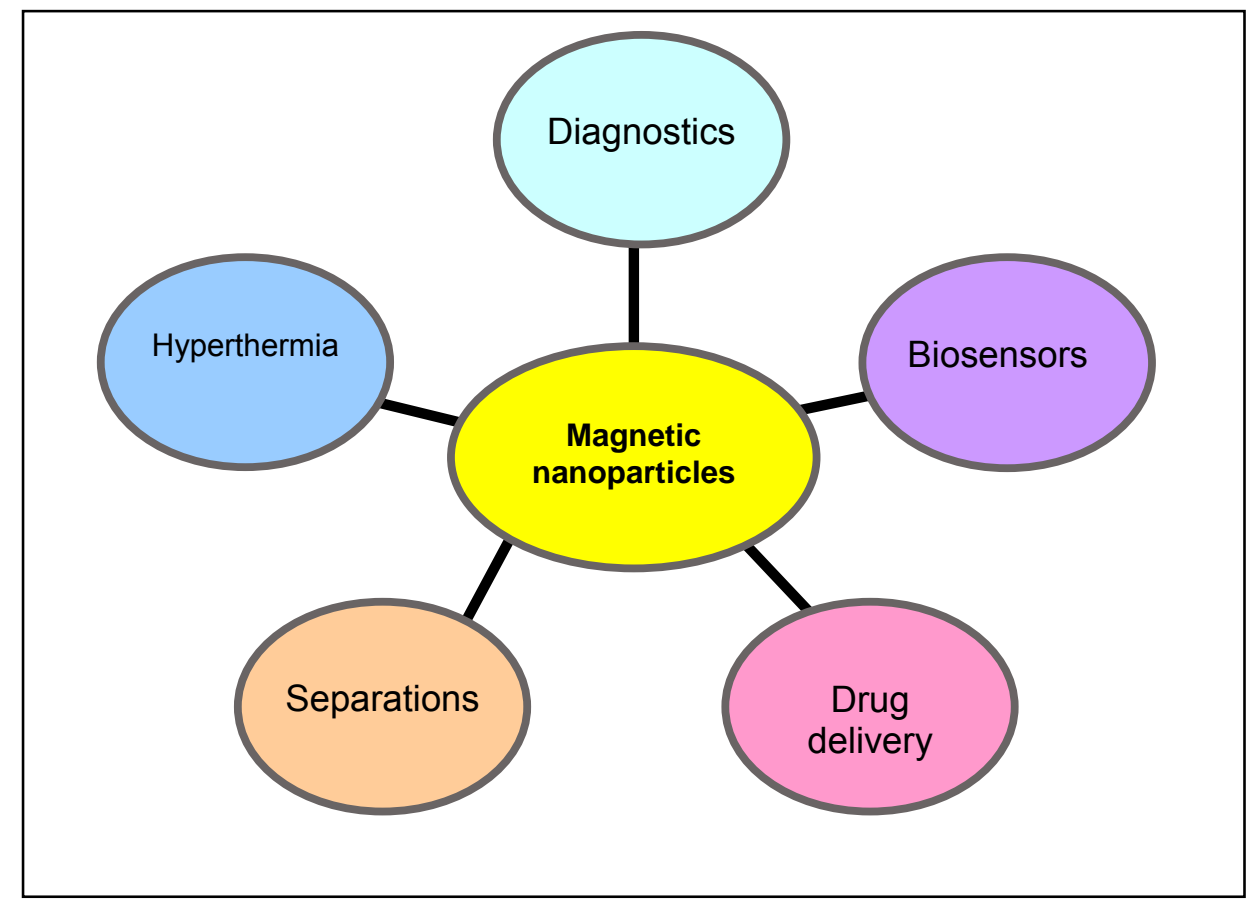

Figure 1-2: Applications of magnetic nanoparticles 


\subsubsection{Drug targeting}

SPIONs can deliver the drug to the site of interest by passive, active and physical targeting. In passive targeting, drug is delivered to the specific sites by the special properties of carriers. In active targeting, drug is delivered to specific sites by special recognition mechanisms. A key advantage to utilizing SPIONs for drug targeting is that in physical targeting, drug is delivered to the specific site by means of external forces such as magnetic field. ${ }^{6}$

Colloidal systems with sizes below $500 \mathrm{~nm}$ are used as drug carrier systems which include microparticles, nano and microemulsions apart from nanoparticles. There are several vital factors such as size, mobility, surface charge, toxicity, density and molecular weight which play an important role in the distribution of these carrier systems within the body system.

\subsubsection{Intake of nanoparticles by cells}

The size of nanoparticles plays an important role in the uptake and elimination of nanoparticles or nano drug delivery vehicles. ${ }^{16}$ The mechanism of cellular uptake of nanoparticles has been investigated previously. Almost all the nanoparticles used for biological applications are hydrophilic and are dispersed in aqueous solutions. These particles cannot pass through the hydrophobic plasma or cell membrane. Nanoparticles are known to be taken up by the cells by endocytosis mechanism. ${ }^{16,17}$ Endocytosis is a process wherein the plasma membrane invaginates to form vesicles that can enclose the extracellular components and 
take them up into the cells. There are several types of endocytosis including receptor mediated endocytosis, phagocytosis (cell eating) and pinocytosis (cell drinking). Reportedly, nanoparticles enter the cells via receptor/clathrin-mediated endocytosis. ${ }^{17}$ In this type of endocytosis, clathrin protein coated pits are formed in the plasma/cell membrane when the target particles bind to the receptor proteins on the plasma membrane and buds to form cytoplasmic vesicles. ${ }^{18}$

\subsubsection{Magnetic Resonance Imaging}

Magnetic resonance imaging is based on the principles of NMR. It is a widely used non-invasive diagnostic method mainly for soft tissues and cartilages based on the difference in relaxation times of hydrogen atoms. SPIONs are widely used as MRI contrast agents due to this difference in hydrogen atom relaxation times. ${ }^{19}$ However, use of SPIONs depends on its physicochemical properties such as size, surface charge and surface coatings. Biocompatible surface coatings such as dextran, starch, poly vinyl acid, polyacrylic acid and poly ethylene glycol have been used on SPIONs. ${ }^{20-23}$ The efficacy of SPIONs as MRI contrast agents can be increased by using biologically active ligands like antibodies, DNA and proteins for surface modification. Currently, a number of SPIONs exist in clinical trials or involved in experimental study stages, examples including Lumiren $®$ for bowel imaging, Feridex IV® for liver and spleen imaging and Combidex® for lymph node metastases imaging. ${ }^{24}$ 


\subsubsection{Hyperthermia}

SPIONs are used in the hyperthermic treatment of tumors. The exposure of SPIONs to alternating magnetic fields induces oscillation of magnetic moment in the particles; magnetic field energy is transformed into heat and liberated into tissue environment. This heating at high temperature $\left(42-46{ }^{\circ} \mathrm{C}\right)$ causes irreversible damage to tumor cells as opposed to reversible damage of healthy cells. Hence, hyperthermia is very useful cancer treatment. Microwaves, ultrasound, infrared and radiofrequency were successfully used conventionally for hyperthermia treatment. However, these techniques lack selectivity and could not induce heat in a specific tissue and temperature distribution is inhomogeneous. SPIONs, due to their smaller size, are absorbed into cells at a higher rate and homogenously distributed in the tissue.

\subsubsection{Biological molecule separations}

Magnetic properties of the magnetic nanoparticles in the presence of external magnetic field made them as attractive tools for bioseparations. ${ }^{25-28}$ Magnetic nanoparticles have been widely used in purification processes and immunoassays. Magnetic micro and nanoparticles were used for effective immobilization and separation of several biomolecules. ${ }^{29,} 30$ Due to their small size, magnetic nanoparticles can be effectively used as "mobile substrates" in bio-assays. They can also be conveniently incorporated into micro and nanofluidic devices. ${ }^{25}$ Magnetic particle based biosensors are widely used for detection of small quantities of biomarkers in clinical diagnostics. ${ }^{31}$ Magnetic 
particles are coupled to antibody or aptamer for target binding. They provide efficient target capture and convenient separation. Magnetic field flow fractionation(MFFF) and electrical field flow fractionation (EIFFF) have been used for separation of variable size magnetic nanoparticles. ${ }^{32}$ Wang et al. showed the use of superparamagnetic microspheres in affinity adsorption of glutathione, a tripeptide found in mammals. ${ }^{33}$

Magnetic particles help in pre-concentration and effective separation of analytes. They also offer advantages over filtration, centrifugation and solid-phase extraction. Processes using magnetic particles are less tedious and less time consuming. Magnetic micro and nanoparticles with high surface area account for minimum steric hindrance, effective surface reaction efficiencies and improved capture efficiency. Magnetic separation is simple and involves application of high magnetic field strength to separate the magnetic particles from its carrier solution. However, time required for magnetic separation depends upon the size and stability of magnetic nanoparticles in solution as well as strength of the applied magnetic field.

Okamoto et al. reported the use of magnetic microspheres for online preconcentration and selective separation of lipoproteins by affinity $C E{ }^{34}$ In this work, multifunctional magnetic microspheres (MFMPs) with a coating of cationic fluorescent tag and anionic affinity ligands on the surface are used. MFMPs are mixed with sample analyte and injected into the capillary as a plug. Online 
trapping and preconcentration of analytes is achieved by applying a magnetic field on the capillary. On removing the magnetic field, the analytes move towards the detector window and detected by laser induced fluorescence (LIF) detector. The concept of online preconcentration and selective separation using MFMPs is shown in figure 1-3. 
1) Immobilization of target analytes onto MFMP surface

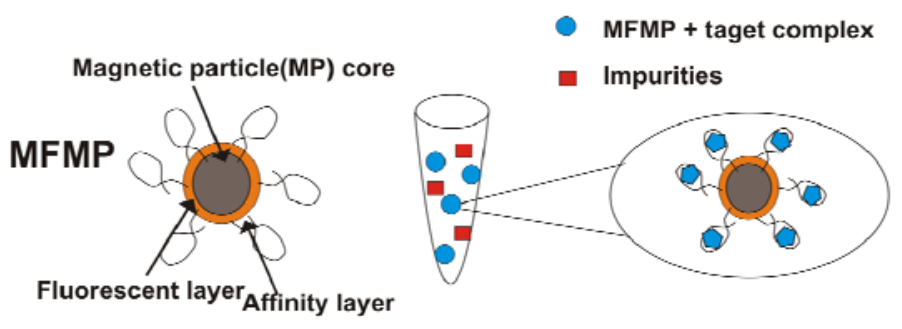

2) Sample injection as a long plug

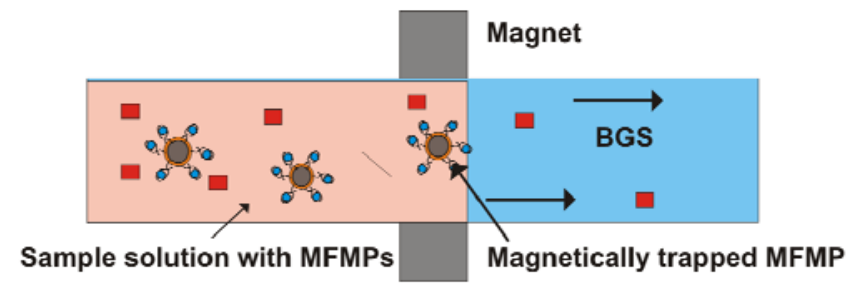

3) Concentration and separation of MFMPs

$(-)$

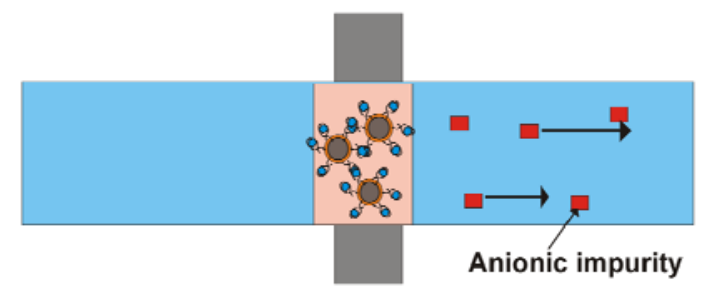

4) Removal of magnetic field

$(-)$

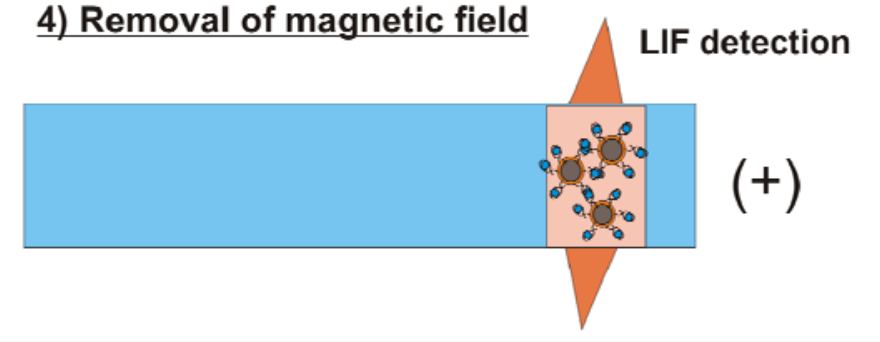

Figure 1-3: Schematic of online preconcentration and selective separation of lipoproteins using multifunctional magnetic particles (MFMPs) in affinity CE (adapted from reference 28). 
Magnetic micro and nanoparticles due to their high surface area enhance the specific interaction of affinity ligands (coated on their surface) with their targets, thus, helping in analyzing trace amounts of analytes. In my research work, I demonstrated the use of SPIONs as analytical sensing elements. I have also made a comparison between the efficiency of magnetic microspheres and nanoparticles.

\subsection{Objectives of my research:}

My research mainly focuses on synthesis of monodisperse SPIONs, surface coating of SPIONs with biocompatible polymers, and attaching biomolecules. These SPIONs are characterized using various analytical techniques such as transmission electron microscope (TEM), Fourier transform infrared spectroscopy (FTIR), Dynamic light scattering (DLS), x-ray diffraction (XRD) and UV-Visible spectroscopy (UV-Vis). SPIONs are also demonstrated as excellent capture elements/ immobilization tools for analytical separations. 


\section{References:}

1. Nakanishi, H.; Bishop, K. J. M.; Kowalczyk, B.; Nitzan, A.; Weiss, E. A.; Tretiakov, K. V.; Apodaca, M. M.; Klajn, R.; Stoddart, J. F.; Grzybowski, B. A., Photoconductance and inverse photoconductance in films of functionalized metal nanoparticles. Nature 2009, 460, (7253), 371-375.

2. Poizot, P.; Laruelle, S.; Grugeon, S.; Dupont, L.; Tarascon, J. M., Nanosized transition-metal oxides as negative-electrode materials for lithium-ion batteries. Nature 2000, 407, (6803), 496-499.

3. Redl, F. X.; Cho, K. S.; Murray, C. B.; O'Brien, S., Three-dimensional binary superlattices of magnetic nanocrystals and semiconductor quantum dots. Nature 2003, 423, (6943), 968-971.

4. Berry, C. C.; Curtis, A. S. G., Functionalisation of magnetic nanoparticles for applications in biomedicine. Journal of Physics D-Applied Physics 2003, 36, (13), R198-R206.

5. Brigger, I.; Dubernet, C.; Couvreur, P., Nanoparticles in cancer therapy and diagnosis. Advanced Drug Delivery Reviews 2002, 54, (5), 631-651.

6. Neuberger, T.; Schopf, B.; Hofmann, H.; Hofmann, M.; von Rechenberg, B., Superparamagnetic nanoparticles for biomedical applications: Possibilities and limitations of a new drug delivery system. Journal of Magnetism and Magnetic Materials 2005, 293, (1), 483-496.

7. Allen, T. M., Ligand-targeted therapeutics in anticancer therapy. Nature Reviews Cancer 2002, 2, (10), 750-763. 
8. Lee, J. H.; Schneider, B.; Jordan, E. K.; Liu, W.; Frank, J. A., Synthesis of complexable fluorescent superparamagnetic iron oxide nanoparticles (FL SPIONs) and cell labeling for clinical application. Advanced Materials 2008, 20, (13), 2512-+.

9. Tan, J. S.; Butterfield, D. E.; Voycheck, C. L.; Caldwell, K. D.; Li, J. T., Surface Modification of Nanoparticles by Peo Ppo Block-Copolymers to Minimize Interactions with Blood Components and Prolong Blood-Circulation in Rats. Biomaterials 1993, 14, (11), 823-833.

10. Meng, J.; Fan, J.; Galiana, G.; Branca, R. T.; Clasen, P. L.; Ma, S.; Zhou, J.; Leuschner, C.; Kumar, C.; Hormes, J.; Otiti, T.; Beye, A. C.; Harmer, M. P.; Kiely, C. J.; Warren, W.; Haataja, M. P.; Soboyejo, W. O., LHRH-functionalized superparamagnetic iron oxide nanoparticles for breast cancer targeting and contrast enhancement in MRI. Materials Science \& Engineering C-Biomimetic and Supramolecular Systems 2009, 29, (4), 1467-1479.

11. Gupta, A. K.; Gupta, M., Synthesis and surface engineering of iron oxide nanoparticles for biomedical applications. Biomaterials 2005, 26, (18), 39954021.

12. Hyeon, T.; Lee, S. S.; Park, J.; Chung, Y.; Bin Na, H., Synthesis of highly crystalline and monodisperse maghemite nanocrystallites without a size-selection process. Journal of the American Chemical Society 2001, 123, (51), 1279812801.

13. Wang, Y.; Wong, J. F.; Teng, X. W.; Lin, X. Z.; Yang, H., "Pulling" nanoparticles into water: Phase transfer of oleic acid stabilized monodisperse 
nanoparticles into aqueous solutions of alpha-cyclodextrin. Nano Letters 2003, 3 , (11), 1555-1559.

14. Elaissari, A.; Fessi, H., Advances in the Biomedical Applications of Reactive Colloids. Brazilian Journal of Physics 2009, 39, (1A), 146-149.

15. Aguilar-Arteaga, K.; Rodriguez, J. A.; Barrado, E., Magnetic solids in analytical chemistry: A review. Analytica Chimica Acta 2010, 674, (2), 157-165.

16. Chithrani, B. D.; Chan, W. C. W., Elucidating the Mechanism of Cellular Uptake and Removal of Protein-Coated Gold Nanoparticles of Different Sizes and Shapes. Nano Letters 2007, 7, (6), 1542-1550.

17. Oh, J.-M.; Choi, S.-J.; Kim, S.-T.; Choy, J.-H., Cellular Uptake Mechanism of an Inorganic Nanovehicle and Its Drug Conjugates:Enhanced Efficacy Due To Clathrin-Mediated Endocytosis. Bioconjugate Chemistry 2006, 17, (6), 14111417.

18. Marsh, M.; Helenius, A., Virus Entry: Open Sesame. Cell 2006, 124, (4), 729-740.

19. Pouliquen, D.; Lejeune, J. J.; Perdrisot, R.; Ermias, A.; Jallet, P., IronOxide Nanoparticles for Use as an Mri Contrast Agent - Pharmacokinetics and Metabolism. Magnetic Resonance Imaging 1991, 9, (3), 275-283.

20. Wu, W.; He, Q.; Jiang, C., Magnetic Iron Oxide Nanoparticles: Synthesis and Surface Functionalization Strategies. Nanoscale Research Letters 2008, 3, (11), 397-415.

21. Cheng, F. Y.; Su, C. H.; Yang, Y. S.; Yeh, C. S.; Tsai, C. Y.; Wu, C. L.; Wu, M. T.; Shieh, D. B., Characterization of aqueous dispersions of Fe3O4 
nanoparticles and their biomedical applications. Biomaterials 2005, 26, (7), 729738.

22. Mahmoudi, M.; Sant, S.; Wang, B.; Laurent, S.; Sen, T., Superparamagnetic iron oxide nanoparticles (SPIONs): Development, surface modification and applications in chemotherapy. Advanced Drug Delivery Reviews 63, (1-2), 24-46.

23. Predoi, D.; Andronescu, E.; Radu, M.; Munteanu, M. C.; Dinischiotu, A., Synthesis and Characterization of Bio-Compatible Maghemite Nanoparticles. Digest Journal of Nanomaterials and Biostructures 2010, 5, (3), 779-786.

24. Veiseh, O.; Gunn, J. W.; Zhang, M. Q., Design and fabrication of magnetic nanoparticles for targeted drug delivery and imaging. Advanced Drug Delivery Reviews 2010, 62, (3), 284-304.

25. Gijs, M. A. M., Magnetic bead handling on-chip: new opportunities for analytical applications. Microfluidics and Nanofluidics 2004, 1, (1), 22-40.

26. Basore, J. R.; Baker, L. A., Applications of microelectromagnetic traps. Analytical and Bioanalytical Chemistry 2012, 403, (8), 2077-2088.

27. Lee, S. Y.; Ahn, C. Y.; Lee, J.; Lee, J. H.; Chang, J. H., Rapid and selective separation for mixed proteins with thiol functionalized magnetic nanoparticles. Nanoscale Research Letters 2012, 7, (1), 279-285.

28. Badruddoza, A. Z. M.; Junwen, L.; Hidajat, K.; Uddin, M. S., Selective recognition and separation of nucleosides using carboxymethyl-beta-cyclodextrin functionalized hybrid magnetic nanoparticles. Colloids and Surfaces BBiointerfaces 2012, 92, 223-231. 
29. Tsai, H. Y.; Hsu, F. H.; Lin, Y. P.; Fuh, C. B., Separation method based on affinity reaction between magnetic and nonmagnetic particles for the analysis of particles and biomolecules. Journal of Chromatography A 2006, 1130, (2), 227231.

30. Tamer, U.; Gundogdu, Y.; Boyaci, I. H.; Pekmez, K., Synthesis of magnetic core-shell $\mathrm{Fe}(3) \mathrm{O}(4)-\mathrm{Au}$ nanoparticle for biomolecule immobilization and detection. Journal of Nanoparticle Research 2010, 12, (4), 1187-1196.

31. Zhang, Y.; Zhou, D. J., Magnetic particle-based ultrasensitive biosensors for diagnostics. Expert Review of Molecular Diagnostics 2012, 12, (6), 565-571.

32. Stephens, J. R.; Beveridge, J. S.; Williams, M. E., Analytical methods for separating and isolating magnetic nanoparticles. Physical Chemistry Chemical Physics 2012, 14, (10), 3280-3289.

33. Wang, Q.; Guan, Y. P.; Yang, M. Z., Application of superparamagnetic microspheres for affinity adsorption and purification of glutathione. Journal of Magnetism and Magnetic Materials 2012, 324, (20), 3300-3305.

34. Okamoto, Y.; Kitagawa, F.; Otsuka, K., Online Concentration and Affinity Separation of Biomolecules Using Multifunctional Particles in Capillary Electrophoresis under Magnetic Field. Analytical Chemistry 2007, 79, (8), 3041 3047. 


\section{Chapter 2}

Pluronic triblock copolymer-modified mono disperse Superparamagnetic Iron Oxide Nanoparticles (SPIONs) 


\subsection{Introduction}

Superparamagnetic Iron Oxide Nanoparticles (SPIONs) are extensively being studied for cancer therapy due to their use in targeted drug delivery, contrast enhancement in Magnetic Resonance Imaging (MRI) and hyperthermia for localized cell death. ${ }^{1,2}$ In the last two decades, the use of magnetic nanoparticles in biomedical applications has increased significantly especially in the treatment of cancer through tumor targeting., 4

Successful use of nanoparticles in biomedical applications is highly dependent on the stability of these particles under in-vivo conditions. The ability to stabilize and disperse the nanoparticles in an aqueous environment and modify the surface with molecules, proteins or other targeting agents is a crucial step for biomedical applications and bioanalysis. ${ }^{1,5}$ Magnetic nanoparticle mediated drug delivery potentially reduces the drug dose, while improving drug efficiency. These effects are due to the poor lymphatic drainage of the cancer cells resulting in enhanced permeability and a retention affect of nanoparticles at the tumor site (also known as passive targeting). ${ }^{6}$ Surface modification of the nanoparticles with polymers helps to avoid uptake by the reticulo-endothelial system and mononuclear phagocytes ${ }^{7}$ and provides long circulation times of nanoparticles. Apart from drug delivery, magnetic nanoparticles can also be used for simultaneous Magnetic Resonance Imaging and hyperthermia treatment of cancer cells. ${ }^{2,8}$ 
Although there are different methods of synthesizing SPIONs ${ }^{9,10}$, the chemical coprecipitation method using iron salts is the most commonly employed methodology. ${ }^{11}$ However, this method often produces polydisperse materials. ${ }^{11}$ In this study, thermal decomposition of iron pentacarbonyl in the presence of oleic acid as a surfactant was used to synthesize uniform, monodispersed and unaggregated SPIONs to facilitate our study of the effects of polymer modification with the exclusion of structural variation. The particles as-synthesized are hydrophobic, and were modified by adsorption of Pluronic triblock copolymers to facilitate aqueous dispersion and stability. A number of methods have been described in the literature for surface modification of similar SPIONs for targeted drug delivery. ${ }^{12,13}$

Amphiphilic Pluronic tri-block copolymers are approved by FDA for use in humans and are currently being used as a part of drug and gene delivery systems. ${ }^{14-16}$ Commercially available, Pluronic tri-block co-polymers were used here to provide water solubility and prevent aggregation and simultaneously create a hydrophobic region within each SPION-Pluronic conjugate which is capable of holding hydrophobic drug molecules. Pluronics are triblock co-polymer surfactants (PEO-PPO-PEO) with hydrophobic polypropylene oxide (PPO) units sandwiched between hydrophilic polyethylene oxide (PEO) chains. The composition of the Pluronic dictates the surfactant HydrophilicLipophilic Balance (HLB). The HLB value of a surfactant is a semi-empirical measure of the degree to which it is hydrophilic or lipophilic based on emulsification behavior, with higher HLB indicating greater hydrophilic nature. For Pluronics, the relative size of different regions (PEO and PPO) of the copolymer affects the HLB value and a greater 
number of ethylene oxide (EO) units in the Pluronic, yields a higher HLB value. A block diagram of a Pluronic structure is given in Figure 2-1 and a summary of the Pluronics used in this experiment is given below in Table 2-1. 


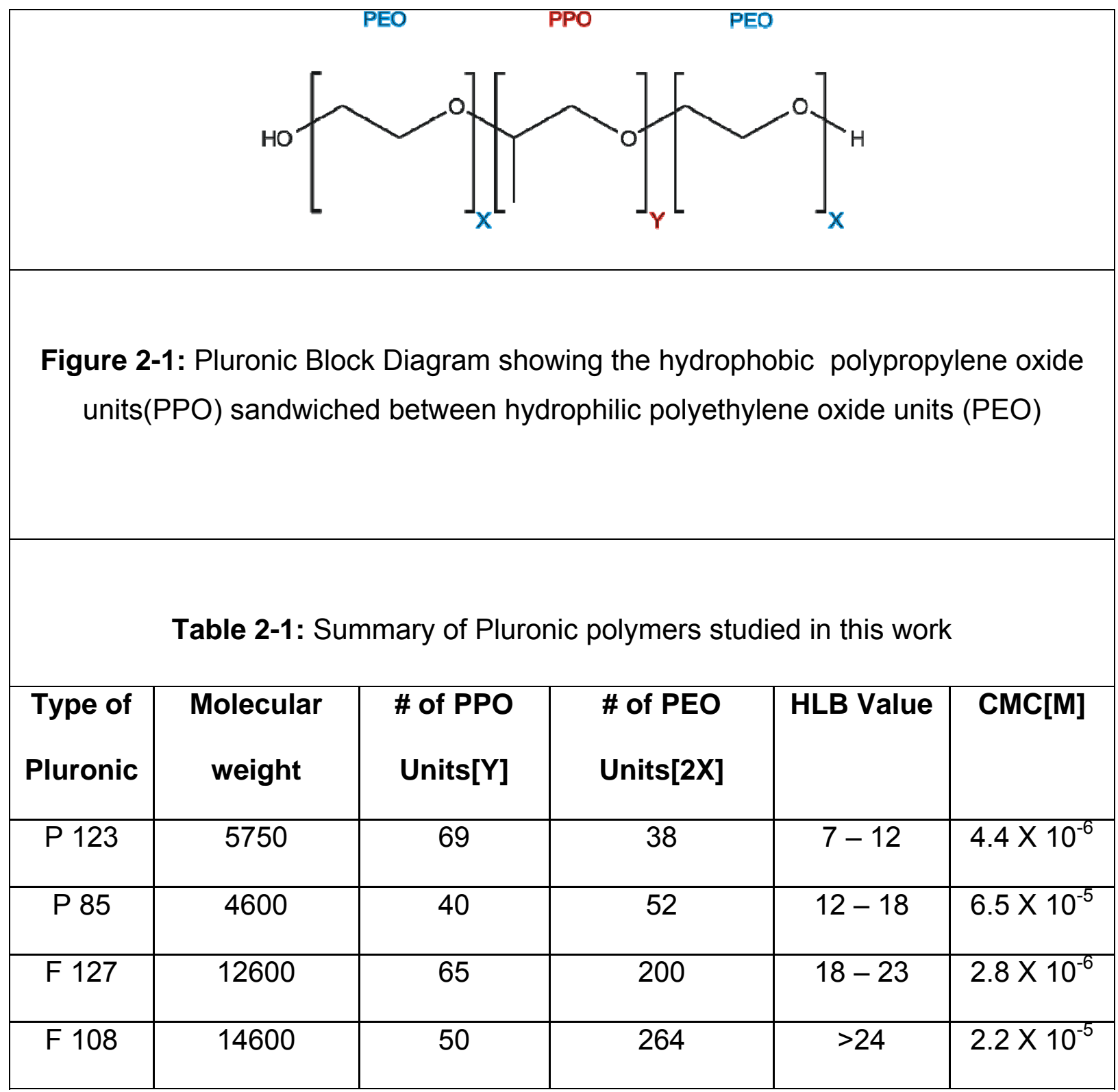


Although Pluronic-coated SPIONs have been studied by Jain and co-workers, ${ }^{8,17,18}$ the SPIONs used were produced by co-precipitation; thus phase transfer is not an issue as the synthesis and modification of SPIONs with polymers both occur in aqueous phase. This could cause variability in SPION stability since the surface of the SPION is hydrophilic and could be expected to interact with the Pluronic differently. Other methods have also been proposed for the phase transfer of SPIONs from organic to aqueous phases ${ }^{19-21}$ which typically resulted in low concentrations of material and there for requiring excessive processing times.

The method described herein is a technically simpler and faster approach of phase transfer for the production of water soluble SPIONs. This approach was used with the selected Pluronics, which covered a wide range of molecular weights and HLB values, to specifically study the physical structure-property relationships in SPIONs. By using the same SPION core throughout this work, the described experiments probed the effect of the Pluronic on the hydrodynamic size of the SPION-Pluronic conjugate and the potential impact on drug delivery. A drug model, the fluorescent dye 1,1'dioctadecyl-3,3,3'3'-tetramethylindocarbocyanine perchlorate (Dil) (chemical structure shown in figure 2-2) was used for determining drug loading capacity, the results of which are reported here. The schematic of dye loaded Pluronic-OA-SPIONs is shown in figure 2-3. 


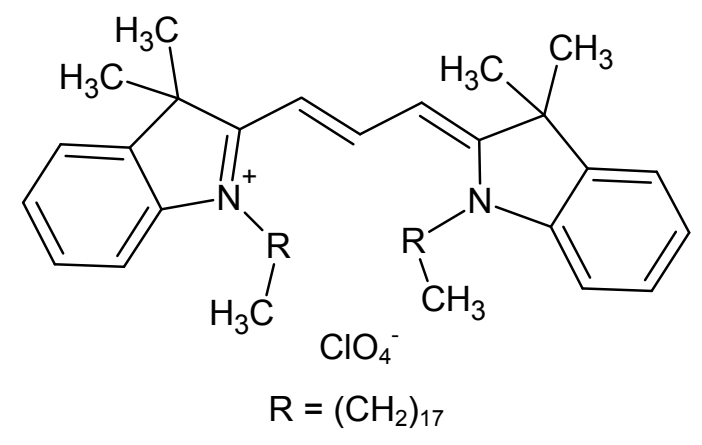

Figure 2-2: Structure of 1,1'-dioctadecyl-3,3,3'3'-tetramethylindocarbocyanine per chlorate (Dil), chemical formula: $\mathrm{C}_{59} \mathrm{H}_{89} \mathrm{ClN}_{2} \mathrm{O}_{4}$, molecular weight : $924.5 \mathrm{~g} / \mathrm{mol}$

OA-SPIONs that were surface coated pluronic were made by a simple and efficient method. Both OA-SPIONs and pluronic-OA-SPIONs were characterized by various analytical techniques so as to evaluate the role of PEO and PPO units of Pluronic polymers on hydrodynamic size and stability. Furthermore, the mechanism of polymer assembly on the SPION surface based on the properties of different Pluronics is hypothesized. 


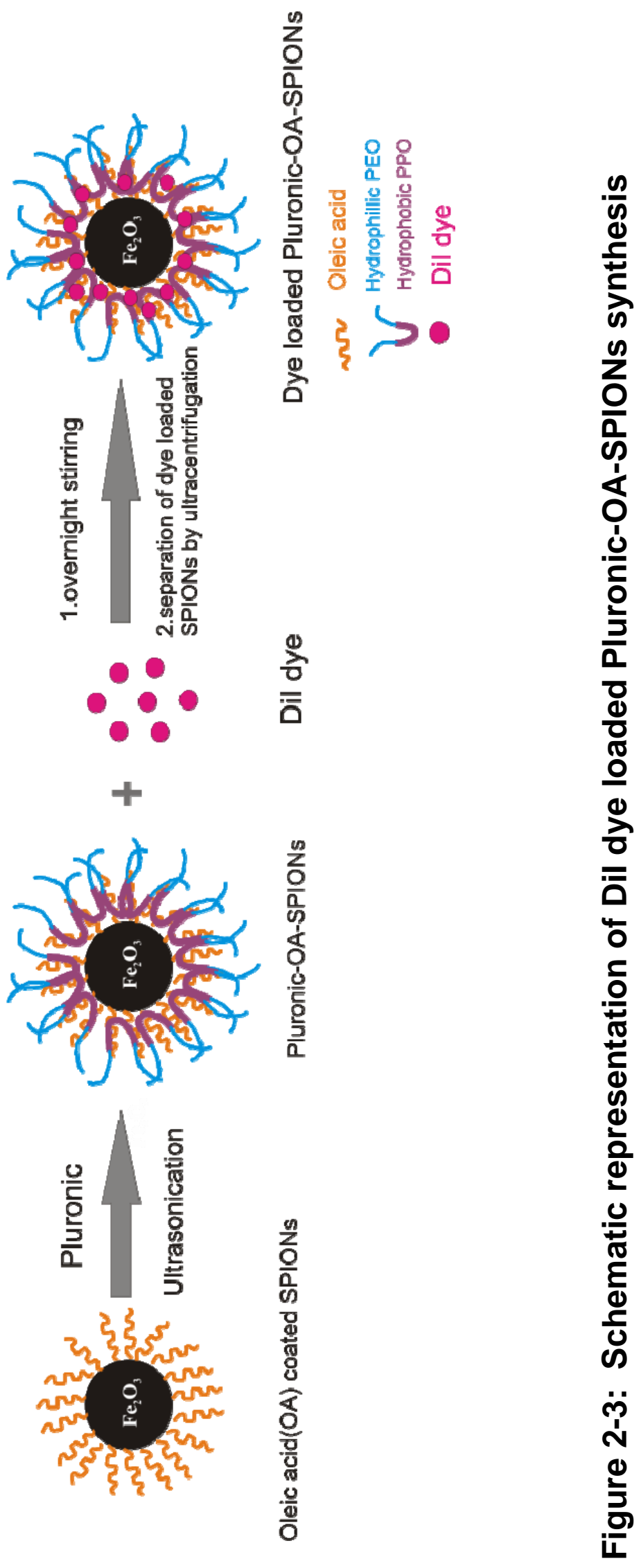

Chapter 2 


\subsection{Experimental}

\subsubsection{Materials and Methods}

\subsubsection{Reagents and materials}

All chemicals including Iron pentacarbonyl, Oleic acid, Trimethylamine $\mathrm{N}$-oxide and 1-1'dioctadecyl-3,3,3'3'-tetramethylindocarbocyanine perchlorate (Dil) -were purchased from Sigma Aldrich (St.Louis, MO, USA). Solvents including toluene, hexane and ethanol were obtained from Fisher Scientific (Pittsburgh, PA, USA). Pluronic samples were obtained as gift from BASF chemical company (Florham Park, NJ, USA).

\subsubsection{Synthesis of monodisperse magnetic nanoparticles by thermal decomposition}

The nanoparticles were synthesized by thermal decomposition method as previously described. ${ }^{9}$ A solution of Oleic acid $(5.12 \mathrm{~g}, 18.1 \mathrm{mmol})$ in octyl ether $(40 \mathrm{~mL})$ was heated at $100^{\circ} \mathrm{C} \mathrm{Fe}(\mathrm{CO})_{5}(0.8 \mathrm{~mL}, 5.93 \mathrm{mmol})$ added the mixture then heated at reflux for $1 \mathrm{~h}$ and then cooled to room temperature. Solid anhydrous trimethylamine $\mathrm{N}$-oxide $(1.36 \mathrm{~g}, 18.1 \mathrm{mmol})$ was then added and the mixture heated at $130{ }^{\circ} \mathrm{C}$ for $2 \mathrm{~h}$ (the reaction mixture becomes brown). The mixture was then heated at reflux for an additional hour (becomes black in appearance). Cooled to room temperature, ethanol added to remove the excess oleic acid, and then this mixture was dispersed in toluene by stirring. The purified SPIONs were easily dispersed in toluene at a concentration of 6 $\mathrm{mg} / \mathrm{mL}$. 


\subsubsection{Preparation of Pluronic coated OA-SPIONs}

Oleic acid-coated SPIONs $(12 \mathrm{mg})$ in toluene $(2 \mathrm{~mL})$ were added to a Pluronic $(48 \mathrm{mg})$ dissolved in distilled water $(8 \mathrm{~mL})$, cooled to $0{ }^{\circ} \mathrm{C}$ and subjected to horn ultrasonication for $30 \mathrm{~min}$. The ultrasonication resulted in an emulsion of toluene in water containing Pluronic-coated SPIONs. The emulsion was broken using ultracentrifugation at 14,000 rpm for $15 \mathrm{~min}$. The aqueous layer turned brown in color, indicating the presence of the Pluronic-coated SPIONs that were carefully separated from the toluene using a syringe. Excess Pluronic in these samples was removed using centrifuge filters with a $100 \mathrm{kDa}$ molecular weight cut-off at 2,000 rpm for $15 \mathrm{~min}$. This purification was repeated three times to ensure the samples were free of excess Pluronic. Pluronic-coated SPIONs were redispersed in deionized water (picture of solutions shown in figure 2-4). These samples were then lyophilized for $24 \mathrm{~h}$ to produce dry powders. 


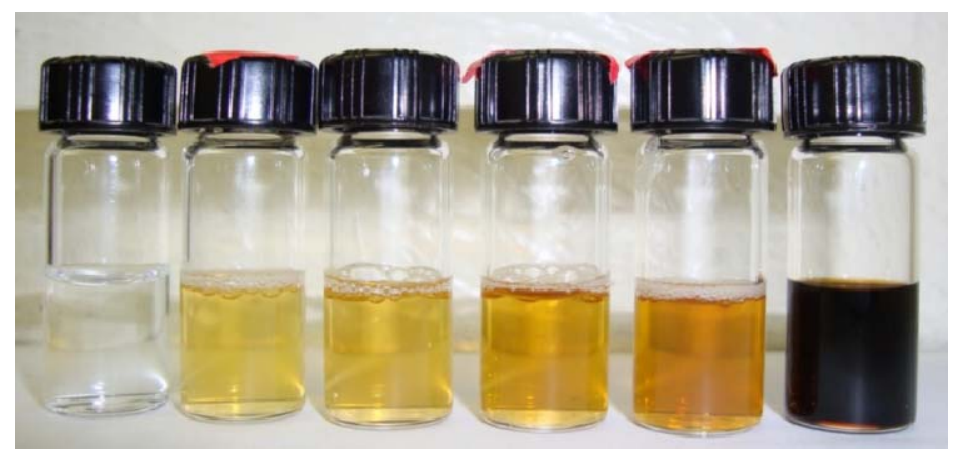

Figure 2-4. From left to right: Water, P 123 coated SPIONs, P 85 coated SPIONs, F 127 coated SPIONs, F 108 coated SPIONs, oleic acid coated SPIONs. All pluronicSPIONs as aqueous dispersions at the same concentration; oleic acid-SPIONs in toluene.

\subsubsection{Loading of Dil dye onto Pluronic coated SPIONs}

The carbocyanine dye, 1,1'-dioctadecyl-3,3,3'3'-tetramethylindocarbocyanine perchlorate (Dil) is a water-insoluble dye used as a drug analogue in our study. A stock solution of $0.2 \mathrm{mg} / \mathrm{mL}$ in DMF was used for all experiments. To the $1.5 \mathrm{~mL}$ of $1 \mathrm{mg} / \mathrm{mL}$ Pluronic coated SPIONs samples, $0.5 \mathrm{ml}$ of $0.2 \mathrm{mg} / \mathrm{mL}$ Dil dye in DMF was added and stirred for $48 \mathrm{~h}$. During this time, the dye molecules partition into the hydrophobic region of the Pluronic-SPIONs defined by the oleic acid and PPO region of the pluronics. The amount of drug entrapped by each SPION is proportional to the hydrophobic region of the particle. After $48 \mathrm{~h}$, the dye-loaded Pluronic-SPIONs were separated from the dye solution using a strong magnet and resuspended in deionized water. Dye-loaded Pluronic-SPIONs dispersed in water were then analysed by absorption spectroscopy at $\lambda_{\mathrm{abs}}=549 \mathrm{~nm}$. Drug entrapment efficiency of the four different Pluronic coated SPIONs 
was calculated using a calibration curve. Entrapment efficiency is given as a ratio of drug/dye loaded onto SPIONs to the total drug/dye used.

\subsubsection{Characterization}

Transmission Electron Microscopy : The morphology and size of the SPIONs was characterized by TEM (JEOL-JEM-2100) operating at an accelerating voltage of $80 \mathrm{kV}$. A drop of the nanoparticle sample was placed on a carbon coated 200 mesh Cu grid sample holder, followed by drying the sample at room temperature.

X-Ray Diffraction: A SPION sample was characterised by room temperature XRD. Diffraction patterns were collected with a Rigaku diffractometer using $\mathrm{CuK}_{\alpha}$ radiation $(\lambda=0.154185 \mathrm{~nm})$ in the scanning range of $2 \theta=15^{\circ}$ to $100^{\circ}$.

Dynamic Light Scattering: Measurements were recorded using a Zetasizer Nano (Malvern UK) at a detection angle of $173^{\circ}$ with a $3 \mathrm{~mW}$ He-Ne laser operating at 633 $\mathrm{nm}$. DLS was used to measure the hydrodynamic diameter of the SPIONs dispersed in toluene and Pluronic coated SPIONs of each kind dispersed in water at $25^{\circ} \mathrm{C}$.

Fourier Transform Infrared Spectroscopy: FTIR Spectroscopy was used to verify functional group modification of SPIONs after synthesis and modification with pluronics. Spectra were collected on samples that were dried on ATR sampling accessory of FTIR (Perkin Elmer) and the percent transmittance spectrum was taken over a range of 4000$650 \mathrm{~cm}^{-1}$. 
Thermogravimetric Analysis: Therrmogravimetry of the SPIONs and Pluronic coated SPIONs was studied by using TGA 7 (Perkin Elmer). Samples were heated to $800^{\circ} \mathrm{C}$ in a stream of Argon gas $(20 \mathrm{ml} / \mathrm{min})$ at a rate of $20^{\circ} \mathrm{C} / \mathrm{min}$ and were held at $800{ }^{\circ} \mathrm{C}$ for 30 $\min$.

Absorption Spectroscopy: Absorption spectra of the dye loaded Pluronic coated SPIONs were obtained using Cary 50 UV-Visible Spectrophotometer. The spectra were recorded from 300 to $800 \mathrm{~nm}$.

\subsection{Results and Discussion}

\subsubsection{Particle Size}

As previously reported by others ${ }^{22}$, the SPIONs prepared by thermal decomposition were well dispersed and monodisperse with an average particle size of $10.1 \pm 0.9 \mathrm{~nm}$ (Figure 2-5a). The size distribution was determined by algorithmic analysis of measurements taken for $\sim 500$ nanoparticles using Image J. SPIONs synthesized by thermal decomposition are highly crystalline as evidenced by HRTEM images shown in inset of Figure 2-5a. Lattice planes are clearly visible and the lattice spacing is consistent with the $<206>$ plane of $\mathrm{y}-\mathrm{Fe}_{2} \mathrm{O}_{3}$, maghemite. From DLS, the hydrodynamic size of the oleic acid-coated SPIONs (OA-SPIONs) is found to be approximately $11.8 \pm$ $0.1 \mathrm{~nm}$. This size is consistent with that determined by TEM, given that TEM neglects the presence of the oleic acid surfactant, and the DLS measurement includes interactions between surfactant and associated solvent molecules. 
A TEM image of Pluronic coated SPIONs is shown in Figure 2-5c and no observable change in crystallinity was observed. An increase in the distances between particles (corresponding to a larger surfactant layer) was not observed between the OA-SPIONs and Pluronic coated nanoparticles. This is because, it is hard to see the organic polymer coating clearly with TEM. However, we could see the formation of cluster like structures with the Pluronic coated SPIONs on the carbon grid indicating the phase transfer of SPIONs. The formvar coated $\mathrm{Cu}$ grid used for the TEM sample preparation is hydrophobic and since the OA-SPIONs in toluene are coated with Pluronics and transferred to aqueous phase, the particles are not spread out uniformly on the grid. That is why the TEM image of Pluronic coated SPIONs were seen as clusters rather than single particles. However, DLS results (Figure 2-8) support that the Pluronic coated SPIONs are not aggregating and are stable. 

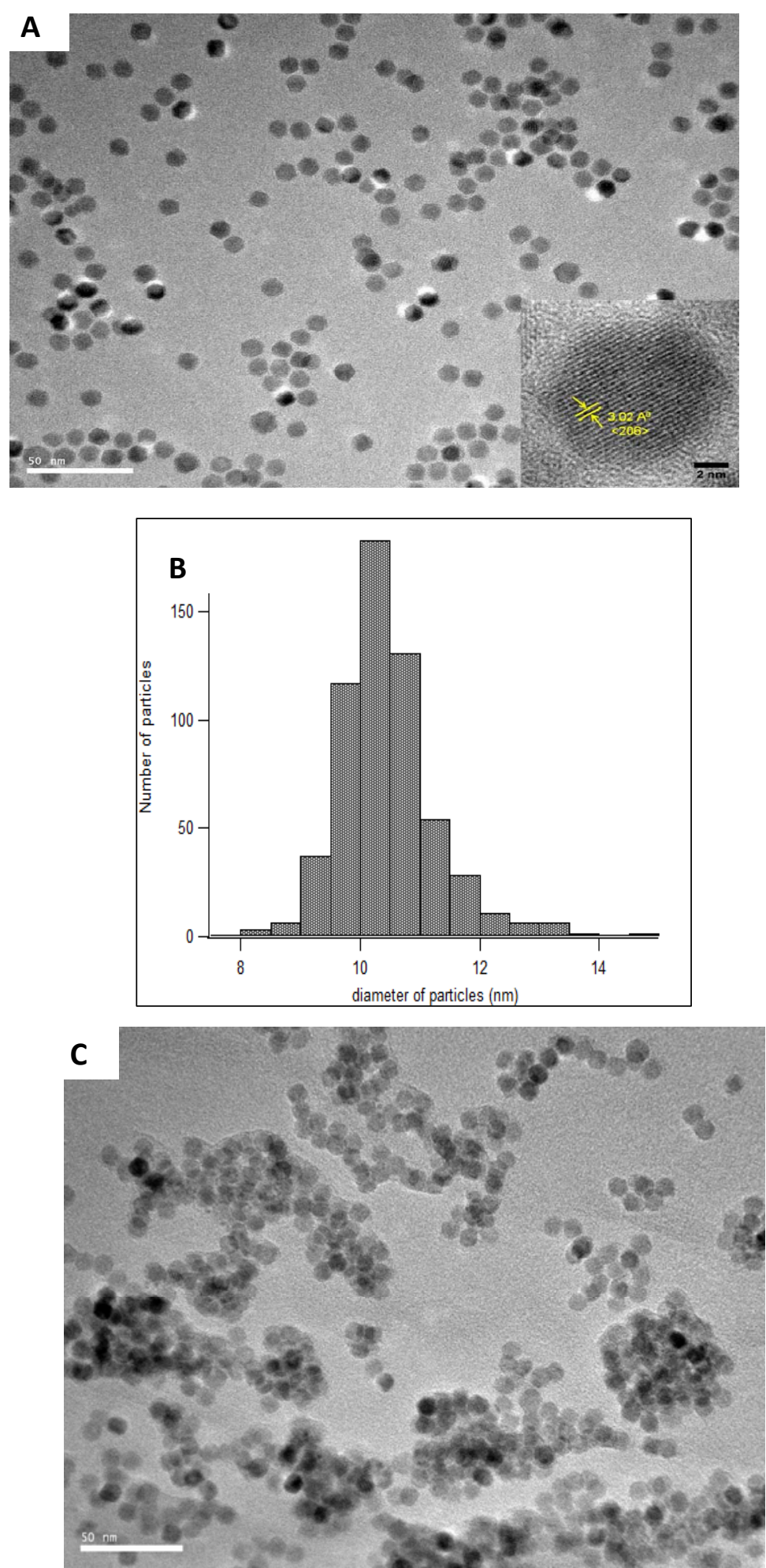

Figure 2-5. a) TEM image of OA-SPIONs in toluene; inset shows HRTEM image of OA-SPIONs showing crystal lattice b) Size distribution of OA-SPIONs by image $J$ c) TEM image of Pluronic-OA-SPIONs 
In figure 2-6, the XRD pattern of the iron oxide nanoparticles is shown. XRD was performed to determine the crystal lattice parameters of the magnetic nanoparticles. $\mathrm{XRD}$ analysis indicates that the synthesized particles were $\mathrm{y}-\mathrm{Fe}_{2} \mathrm{O}_{3}$ (Maghemite) based on the positions of peaks. XRD peak analysis and fitting to the Scherrer equation gave a crystallite size of $10.5 \mathrm{~nm}$ in close agreement with TEM and DLS results. 


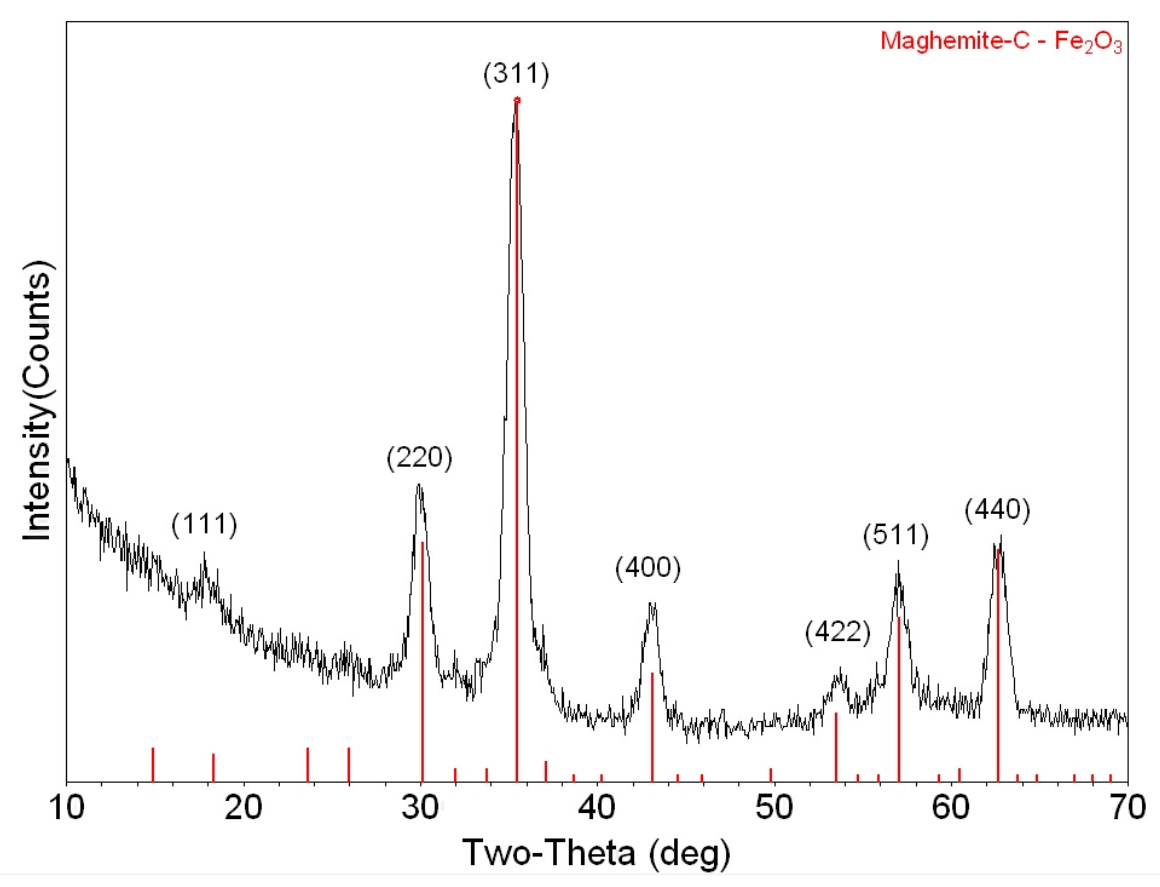

Figure 2-6. XRD patterns of oleic acid coated SPIONs synthesised by thermal decomposition with standard diffraction pattern for maghemite shown by the vertical lines. 


\subsubsection{Pluronic adsorbed on SPIONs}

The preparation technique described here takes advantage of the tendency of the Pluronic block copolymers to adsorb to hydrophobic surfaces through the central PPO block, with the PEO blocks projecting away from the surface. This phenomenon has been extensively studied with respect to Pluronic adsorption on flat surfaces, particles

and vesicles. ${ }^{23-26}$ Here, the adsorption of the Pluronics to the hydrophobic OA-SPIONs imparts aqueous solubility to the SPIONs, and increases the volume of hydrophobic materials at the SPION surface into which hydrophobic drug molecules will selectively partition. Excess Pluronic molecules were removed from the Pluronic SPIONs suspension through the use of centrifuge filters with a molecular weight cutoff of 100 $\mathrm{kDa}$. This is much higher than the molecular weight of even the largest of the Pluronics, and empirical examination showed no passage of Pluronic-SPIONs through the filter. To confirm that the Pluronics were adsorbed onto the SPIONs, the OA-SPIONs and Pluronic-SPIONs were analyzed by FTIR, DLS and TGA.

The FTIR spectra of OA-SPIONs, Pluronic and Pluronic-OA-SPIONs are shown in Figure 2-7. The OA-SPIONs show strong peaks corresponding to methyl/ethylene symmetric/asymmetric stretches at $2750-3000 \mathrm{~cm}^{-1}$ along with carboxylate anion stretches at $1467 \mathrm{~cm}^{-1}$ and $1526 \mathrm{~cm}^{-1}$, confirming the presence of oleic acid on the synthesized particles. After Pluronics were adsorbed onto the exterior of the SPION, the intensity of these peaks is attenuated by the coating of Pluronic co-polymer. All the Pluronic-SPIONs show spectra consistent with free Pluronic in solution shown as a reference confirming the adsorption of pluronics onto SPIONs. 


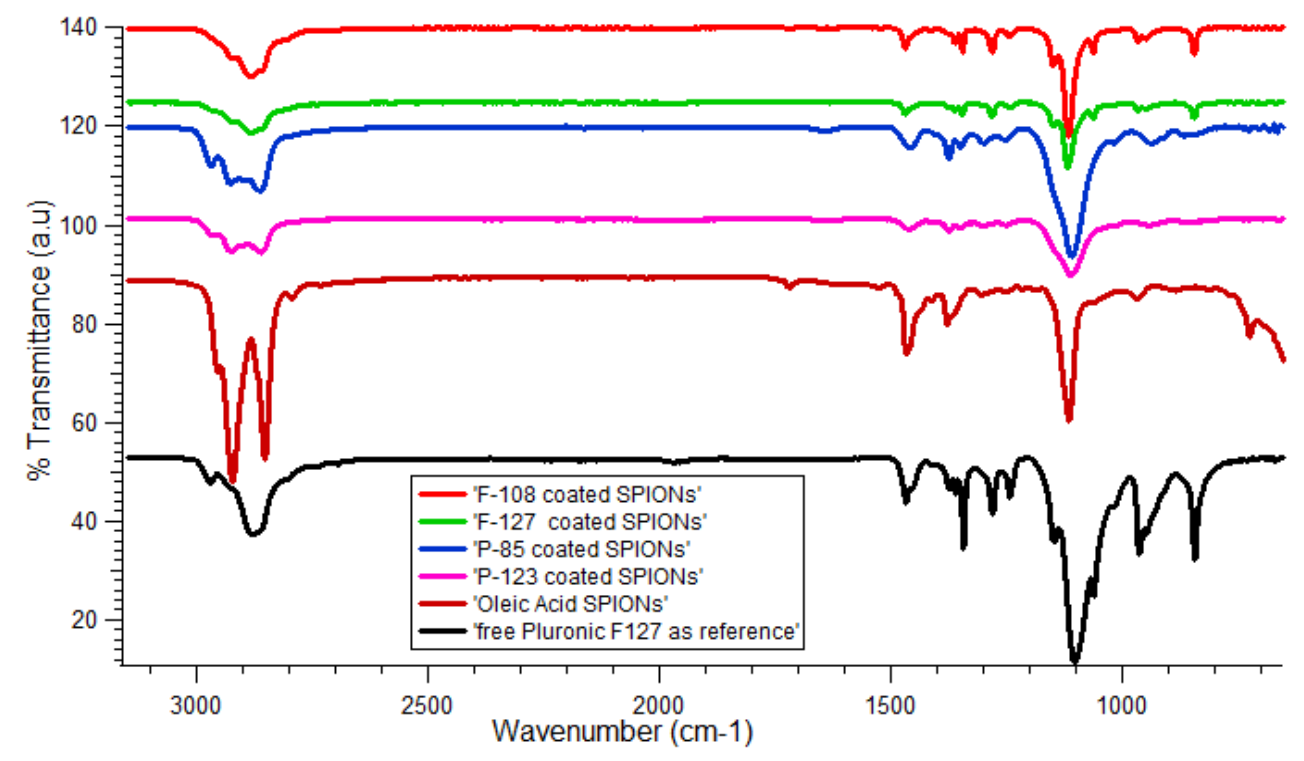

Figure 2-7. FTIR spectra of OA-SPIONs, Pluronic-OA-SPIONs and free pluronic F127 as reference showing the functional groups. 
Pluronics of various sizes were adsorbed to the OA-SPIONs. Two primary possibilities exist for adsorption mechanisms of Pluronics to the hydrophobic OA-SPIONs: 1) the PPO block interacts with the surface of the OA-SPIONs, or 2) the PPO block interpenetrates the OA surfactant layer on the SPIONs. In either case, the adsorption of the Pluronics onto the OA-SPIONs will be self-limiting due to the loss of conformational entropy of the PEO (entropy associated with the compact physical arrangement of PEO chains of polymer in solution).

From the DLS results (shown in figure 2-8), we can observe that with the increase in molecular weight of the Pluronics and the PEO subunits, the hydrodynamic size of the pluronic-SPIONs increases. However, we could see a discrepancy in the trend with $\mathrm{P}$ 123. Though $P 123$ has fewer PEO units, it has higher hydrodynamic size than $F 127$. This might have been due to the formation of micelles. We observed almost similar results with these systems when checked after 6 months for their stability over time. This reveals that this novel method followed for the phase transfer of SPIONs results in stable systems. This will be a great advantage in terms of the formulation shelf life in future. 

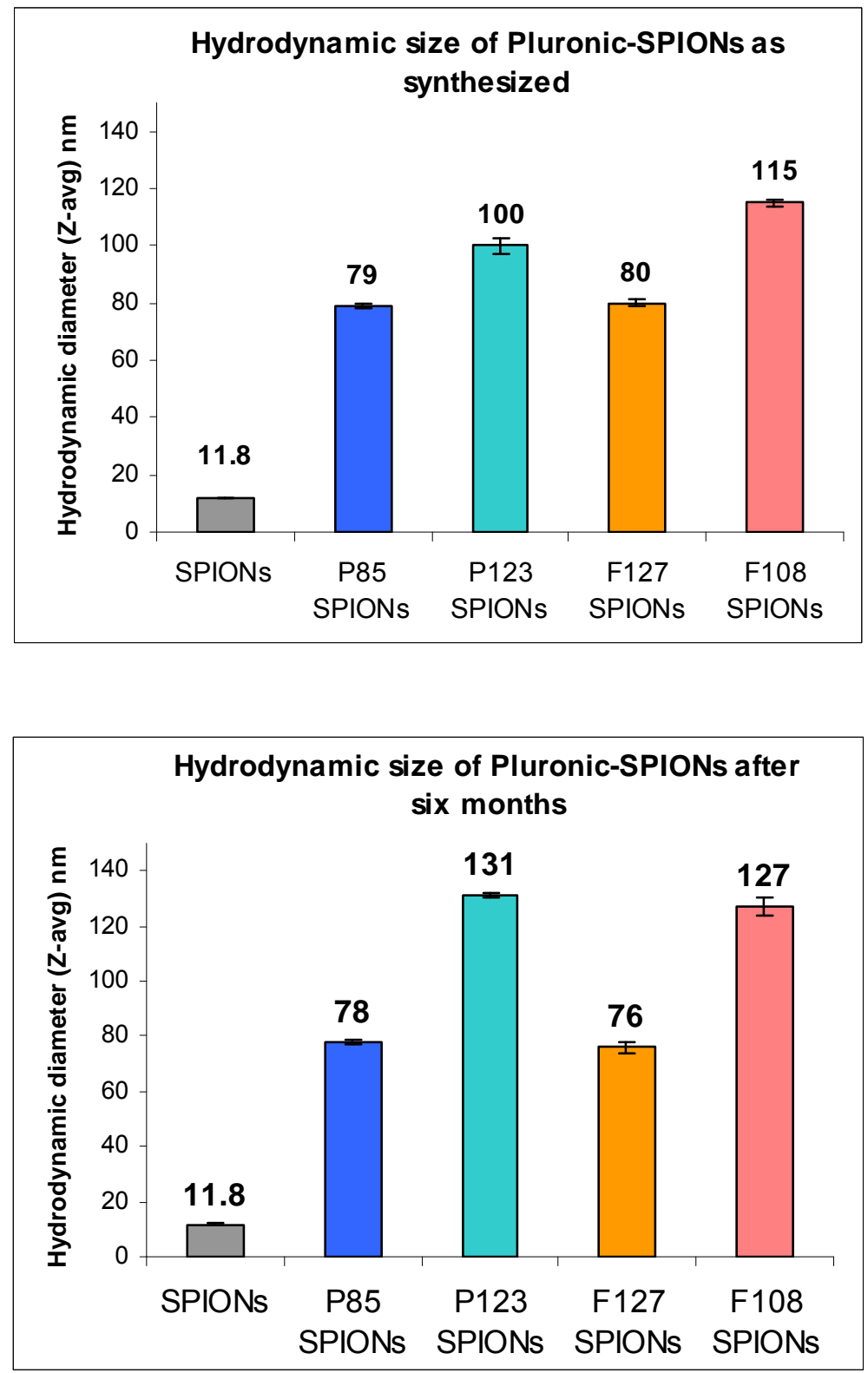

Figure 2-8. a) Graphical Representation of hydrodynamic size variation of Pluronic coated OA-SPIONs before b) after 6 months 
Thermo gravimetric analysis was used to understand the mass relationship between the surfactant layer and iron oxide core of the particles allowing estimation of the surface coverage for each system. All the Pluronic-coated SPION samples began losing mass at $150{ }^{\circ} \mathrm{C}$ and stabilized by $\sim 500{ }^{\circ} \mathrm{C}$ as seen in Figure $2-9$ as determined from the first derivative curves of TGA data. Oleic acid-coated SPIONs lost mass beginning $\sim 220^{\circ} \mathrm{C}$. Assuming that the Pluronic block co-polymer is desorbed from $200^{\circ} \mathrm{C}$ to $500^{\circ} \mathrm{C}$, the percent coating on the SPIONs was calculated and is shown in Table 2-2. From consideration of the TGA data of the OA-SPIONs (Figure 2-9), oleic acid represents $22.6 \%$ of the total coating in all the samples and the remaining percentage of coating is the Pluronic. These results suggest that the P123 and F127 SPIONs are coated well with the Pluronic followed by F108 and P85 in agreement with the DLS data. 


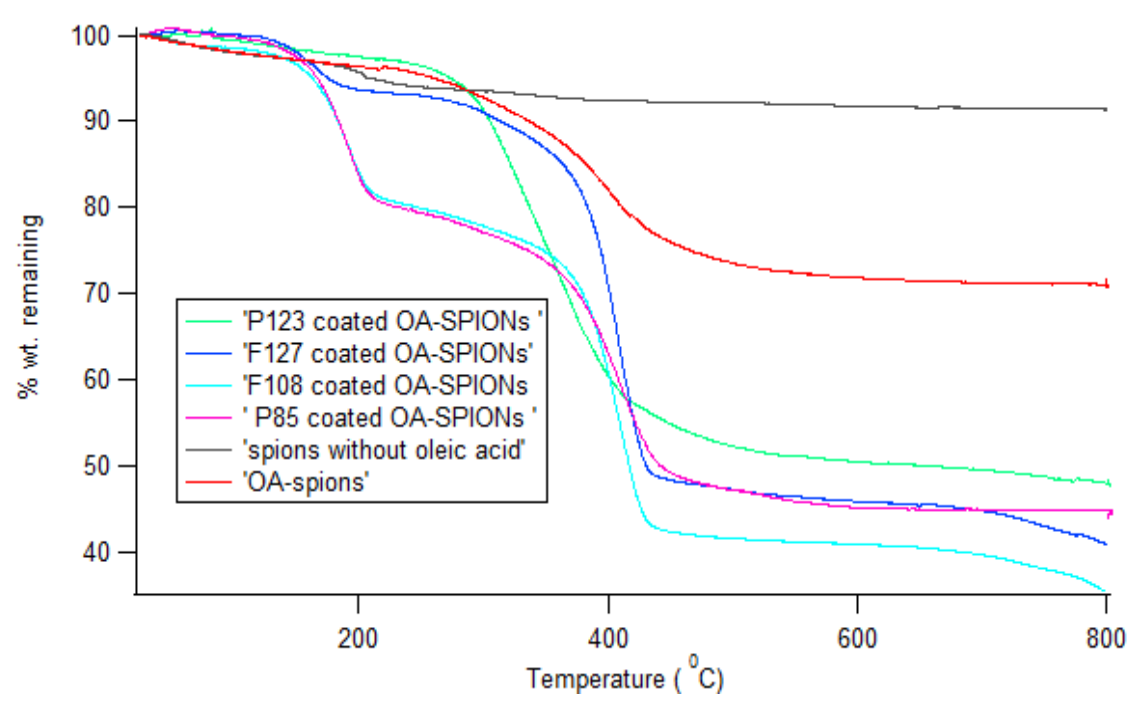

Figure 2-9. TGA of SPIONs and Pluronic coated SPIONs showing the degradation of organic polymer at high temperatures

Table 2-3. Analysis of TGA results

\begin{tabular}{|c|c|c|c|c|c|}
\hline $\begin{array}{l}\text { Type of } \\
\text { Pluronic }\end{array}$ & $\begin{array}{c}\text { Temperature } \\
\text { (T1 in } \\
\text { Celsius) }\end{array}$ & $\begin{array}{c}\text { \%wt. } \\
\text { remaining at } \\
\text { T1 }\end{array}$ & $\begin{array}{c}\text { Temperature } \\
\text { (T2 in } \\
\text { Celsius) }\end{array}$ & $\begin{array}{c}\text { \%wt. } \\
\text { remaining } \\
\text { at T2 }\end{array}$ & $\begin{array}{c}\% \text { coating } \\
\text { on } \\
\text { SPIONs }\end{array}$ \\
\hline OA-SPIONs & 205.14 & 96.22 & 500.09 & 73.58 & 22.65 \\
\hline P85 & 205.46 & 82.16 & 500.60 & 47.28 & 34.87 \\
\hline P123 & 205.32 & 97.39 & 500.33 & 52.32 & 45.06 \\
\hline F127 & 205.40 & 93.58 & 500.41 & 47.27 & 46.31 \\
\hline F108 & 205.23 & 82.67 & 500.52 & 41.64 & 41.03 \\
\hline
\end{tabular}


Though P123 is the smaller molecule (MW 5750 AMU) the TGA results of P123SPIONs suggest that it behaves similar to that of F127-SPIONs i.e. it showed similar surface coverage as that of F127. Both F127 and P123 have equivalent percent weights of Pluronic coating as per the TGA analysis from Table 2-2. This can be attributed to their similar number of PPO groups (69 in P123 and 65 in F 127) that helps in the attachment of Pluronics to the SPIONs. As expected, P85 with fewest number of PPO units and low molecular weight showed the smallest percentage of Pluronic coating on SPIONs, which supporting the proposed role of PPO in the adsorption of pluronic onto SPION. F108-SPIONs showed a higher percentage of Pluronic coating compared to P85-SPIONs and lesser coating compared to P123 \& F127-SPIONs also supporting the role of PPO in the adsorption of Pluronic on SPION.

\subsubsection{Loading of hydrophobic dye Dil onto Pluronic coated SPIONs}

Absorption spectroscopy was used to determine the absorbance of dye encapsulated by Pluronic-OA-SPIONs and all the samples showed a distinct absorption double peak similar to that of standard solutions of dye (shown in Figure 2-10a). Since the $\lambda_{\max }$ of the dye adsorbed onto SPIONs has shifted, absorbance at $590 \mathrm{~nm}$ is taken both for Pluronic-SPIONs and dye loaded Pluronic SPIONs. Here, we are assuming that the molar absorptivity of the dye remains the same even on the nanoparticle surface, which may not be true. Hence, the calculated values (based on background subtracted absorbance) of loading efficiency can be slightly different. However, the qualitative trend 
shown remains the same. A calibration curve was derived from carbocyanine dye solutions of known concentrations yielding a linear fit of $y=m x+c$. Percent entrapment efficiency of the Pluronic coated SPIONs was calculated from the calibration curve.

\% Entrapment or loading efficiency is given by:

(Dye loaded onto SPIONs $(\mu \mathrm{g} / \mathrm{mL}) /$ Total dye used for loading $(\mu \mathrm{g} / \mathrm{mL})) * 100$

Refer to the sample calculation is given on pg. 66 in chapter 3.

The comparison of hydrophobic dye entrapment efficiency of OA-Pluronic SPIONs is shown in Figure 2-10b. The drug encapsulation efficiency increased with the increase in molecular weight and size. SPIONs coated with Pluronic F108 showed the highest dye entrapment efficiency followed by the Pluronic F127. Thus the structural and molecular size properties of the Pluronics on the SPIONs play a major role in the drug storage capacity. The larger entrapments are attributed to larger hydrophobic regions being created in the polymer layer as would be expected from system involving longer PPO chains. 

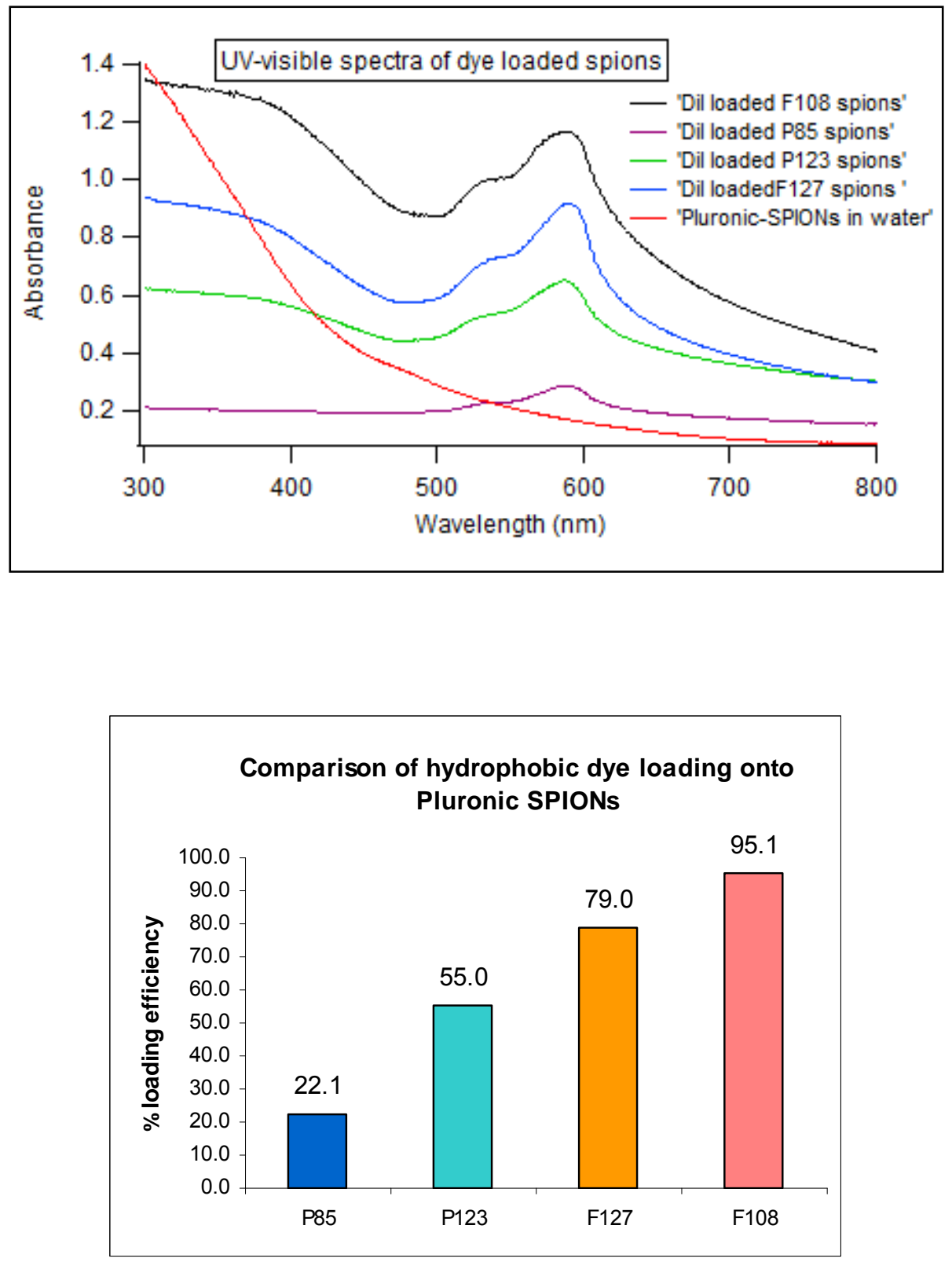

Figure 2-10. a) UV-Visible absorbance of OA-Pluronic SPIONs with entrapped Dil dye

b) Comparison of hydrophobic dye loading efficiency of OA-Pluronic SPIONs 


\subsection{Conclusions \& Future directions}

A simple, novel and fast approach of coating Pluronics onto uniform monodisperse SPIONs has been developed and has proved to be successful in producing potentially biocompatible aqueous solutions of maghemite. This study on hydrodynamic diameter variations and thermo gravimetric analysis of various Pluronic coated SPIONs clearly indicated that the structure and molecular weight of Pluronics play a major role in the attachment of these polymers onto SPIONs and also demonstrated their effect on drug analogue entrapment efficiency based on the absorbance data.

These absorbance measurements and comparison of drug loading efficiencies can be considered as qualitative or semi quantitative. Quantitative measurements can be achieved by separating SPIONs from supernatant using ultracentrifugation and then measuring the drug in both fractions. In this method, if the molar absorptivity $(\varepsilon)$ of the dye is affected by interaction with the SPION coating the absorbance can be determined by the method of standard addition. If this is not feasible e.g. due to dye saturation, then after separating the SPIONs from supernatant the dye can be released in an organic solvent, recovered and reconstituted in a solvent equivalent to the supernatant and measured with UV-Vis absorbance.

Pluronic F127 and F108 were found to be the most efficient of polymer coatings for drug encapsulation studied here. The comparison of entrapment efficiency of these Pluronic SPIONs will give an idea on how much of hydrophobic drug could be loaded. 
However, release and bioavailability of drug in the biological environments is more important for its in vivo applications. Now that the entrapment efficiency of Pluronic SPIONs is characterized, the next step towards the clinical development is to study the drug release properties in-vitro from these systems produced by the new approach described here.

Tri block co-polymers Pluronics are commercially available and are FDA approved. Pluronic coated oleic acid-SPIONs can be used for loading hydrophobic drugs for targeted drug delivery by passive targeting. However, Pluronic polymers lack a functional group useful for conjugating biomolecules and cannot achieve active targeting. 


\section{References}

1. Chouly, C.; Pouliquen, D.; Lucet, I.; Jeune, J. J.; Jallet, P., Development of superparamagnetic nanoparticles for MRI: Effect of particle size, charge and surface nature on biodistribution. Journal of Microencapsulation 1996, 13, (3), 245-255.

2. Yallapu, M. M.; Othman, S. F.; Curtis, E. T.; Gupta, B. K.; Jaggi, M.; Chauhan, S. C., Multi-functional magnetic nanoparticles for magnetic resonance imaging and cancer therapy. Biomaterials 2010, 32, (7), 1890-1905.

3. Brannon-Peppas, L.; Blanchette, J. O., Nanoparticle and targeted systems for cancer therapy. Advanced Drug Delivery Reviews 2004, 56, (11), 1649-1659.

4. Brigger, I.; Dubernet, C.; Couvreur, P., Nanoparticles in cancer therapy and diagnosis. Advanced Drug Delivery Reviews 2002, 54, (5), 631-651.

5. LaVan, D. A.; McGuire, T.; Langer, R., Small-scale systems for in vivo drug delivery. Nat Biotech 2003, 21, (10), 1184-1191.

6. Maeda, H.; Sawa, T.; Konno, T., Mechanism of tumor-targeted delivery of macromolecular drugs, including the EPR effect in solid tumor and clinical overview of the prototype polymeric drug SMANCS. Journal of Controlled Release 2001, 74, (1-3), 47-61.

7. Chen, K.; Xie, J.; Xu, H. Y.; Behera, D.; Michalski, M. H.; Biswal, S.; Wang, A.; Chen, X. Y., Triblock copolymer coated iron oxide nanoparticle conjugate for tumor integrin targeting. Biomaterials 2009, 30, (36), 6912-6919.

8. Jain, T. K.; Foy, S. P.; Erokwu, B.; Dimitrijevic, S.; Flask, C. A.; Labhasetwar, V., Magnetic resonance imaging of multifunctional pluronic stabilized iron-oxide nanoparticles in tumor-bearing mice. Biomaterials 2009, 30, (35), 6748-6756. 
9. Massart, R., Preparation of Aqueous Magnetic Liquids in Alkaline and Acidic Media. Ieee Transactions on Magnetics 1981, 17, (2), 1247-1248.

10. Mahmoudi, M.; Sant, S.; Wang, B.; Laurent, S.; Sen, T., Superparamagnetic iron oxide nanoparticles (SPIONs): Development, surface modification and applications in chemotherapy. Advanced Drug Delivery Reviews 63, (1-2), 24-46.

11. Cheng, F. Y.; Su, C. H.; Yang, Y. S.; Yeh, C. S.; Tsai, C. Y.; Wu, C. L.; Wu, M. T.; Shieh, D. B., Characterization of aqueous dispersions of Fe3O4 nanoparticles and their biomedical applications. Biomaterials 2005, 26, (7), 729-738.

12. Mahmoudi, M.; Simchi, A.; Imani, M., Recent Advances in Surface Engineering of Superparamagnetic Iron Oxide Nanoparticles for Biomedical Applications. Journal of the Iranian Chemical Society 7, S1-S27.

13. Gupta, A. K.; Gupta, M., Synthesis and surface engineering of iron oxide nanoparticles for biomedical applications. Biomaterials 2005, 26, (18), 3995-4021.

14. Kwon, G. S.; Kataoka, K., Block copolymer micelles as long-circulating drug vehicles. Advanced Drug Delivery Reviews 1995, 16, (2-3), 295-309.

15. Lee, E. S.; Oh, Y. T.; Youn, Y. S.; Nam, M.; Park, B.; Yun, J.; Kim, J. H.; Song, H.-T.; Oh, K. T., Binary mixing of micelles using Pluronics for a nano-sized drug delivery system. Colloids and Surfaces B: Biointerfaces 2011, 82, (1), 190-195.

16. Wang, Y.; Yu, L.; Han, L.; Sha, X.; Fang, X., Difunctional Pluronic copolymer micelles for paclitaxel delivery: Synergistic effect of folate-mediated targeting and Pluronic-mediated overcoming multidrug resistance in tumor cell lines. International Journal of Pharmaceutics 2007, 337, (1-2), 63-73. 
17. Jain, T. K.; Morales, M. A.; Sahoo, S. K.; Leslie-Pelecky, D. L.; Labhasetwar, V., Iron oxide nanoparticles for sustained delivery of anticancer agents. Molecular Pharmaceutics 2005, 2, (3), 194-205.

18. Jain, T. K.; Richey, J.; Strand, M.; Leslie-Pelecky, D. L.; Flask, C. A.; Labhasetwar, V., Magnetic nanoparticles with dual functional properties: Drug delivery and magnetic resonance imaging. Biomaterials 2008, 29, (29), 4012-4021.

19. Gonzales, M.; Krishnan, K. M., Phase transfer of highly monodisperse iron oxide nanocrystals with Pluronic F127 for biomedical applications. Journal of Magnetism and Magnetic Materials 2007, 311, (1), 59-62.

20. Gonzales, M.; Mitsumori, L. M.; Kushleika, J. V.; Rosenfeld, M. E.; Krishnan, K. M., Cytotoxicity of iron oxide nanoparticles made from the thermal decomposition of organometallics and aqueous phase transfer with Pluronic F127. Contrast Media \& Molecular Imaging 5, (5), 286-293.

21. Wang, Y.; Wong, J. F.; Teng, X. W.; Lin, X. Z.; Yang, H., "Pulling" nanoparticles into water: Phase transfer of oleic acid stabilized monodisperse nanoparticles into aqueous solutions of alpha-cyclodextrin. Nano Letters 2003, 3, (11), 1555-1559.

22. Hyeon, T.; Lee, S. S.; Park, J.; Chung, Y.; Bin Na, H., Synthesis of highly crystalline and monodisperse maghemite nanocrystallites without a size-selection process. Journal of the American Chemical Society 2001, 123, (51), 12798-12801. 23. Kostarelos, K.; Luckham, P. F.; Tadros, T. F., Steric stabilization of phospholipid vesicles by block copolymers - Vesicle flocculation and osmotic swelling caused by monovalent and divalent cations. Journal of the Chemical Society-Faraday Transactions 1998, 94, (15), 2159-2168. 
24. Kostarelos, K.; Tadros, T. F.; Luckham, P. F., Physical conjugation of (tri-) block copolymers to liposomes toward the construction of sterically stabilized vesicle systems. Langmuir 1999, 15, (2), 369-376.

25. Lin, Y. N.; Alexandridis, P., Temperature-dependent adsorption of pluronic F127 block copolymers onto carbon black particles dispersed in aqueous media. Journal of Physical Chemistry B 2002, 106, (42), 10834-10844.

26. Nejadnik, M. R.; Olsson, A. L. J.; Sharma, P. K.; van der Mei, H. C.; Norde, W.; Busscher, H. J., Adsorption of Pluronic F-127 on Surfaces with Different Hydrophobicities Probed by Quartz Crystal Microbalance with Dissipation. Langmuir 2009, 25, (11), 6245-6249. 


\section{Chapter 3}

Antisense Survivin coupled surface modified magnetic nanoparticles for targeted cancer therapy 


\subsection{Introduction}

In the previous chapter SPIONs were synthesized by thermal decomposition and surface modified with tri-block co-polymers, Pluronics. To these surface modified SPIONs, a hydrophobic dye was loaded as a surrogate to drug. However, Pluronics lack the functional groups necessary to attach the biomolecules such as antibodies and nucleic acids that may help in active targeting for drug delivery as well as magnetic resonance imaging. Hence, this chapter demonstrates the possibility of using a co-polymer with a functional group useful for attaching nucleic acid ligands onto SPIONs. An anti cancer drug, epirubicin was also loaded onto these functionalized SPIONs.

Nanoparticles possess distinct physical and chemical properties which make them unique for many applications such as biosensing, drug delivery and separations. ${ }^{1-5}$ Magnetic nanoparticles in particular offer several advantages. Superparamagnetic iron oxide nanoparticles (SPIONs) have been demonstrated to be biocompatible with living systems. Hence SPIONs are under investigation for use in a wide range of human health applications, including targeted drug delivery, MRI contrast enhancement and hyperthermia. ${ }^{6-8}$ To achieve these goals, control of particle structure, function, and stability in physiologically relevant conditions is critical. $^{9}$ 
Currently a number of SPIONs based therapeutics are under investigation, examples include Lumiren $®$ for bowel imaging, Feridex $I V \circledast$ for liver and spleen imaging, Combidex $®$ for lymph node metastases imaging, and Ferumoxytol $₫$ for iron replacement therapy. ${ }^{10}$ However, these SPIONs lack multifunctional properties such as specific targeting of drug delivery or imaging. Though the passive targeting of nanoparticles is possible for using them in cancer therapy, it is neither homogenous in all tissues nor effective in all types of cancer. To overcome this limitation, use of active targeting by functionalizing nanoparticles with different biomolecules is proposed.

DNA sequences that interfere with protein expression (Antisense DNA) are effective in treatment of cancer. ${ }^{11-13}$ Survivin, a protein over expressed in many cancer types, inhibits apoptosis which allows cancer cells to grow unchecked. Survivin has also been shown to be one of the most cancer-specific proteins identified to date, being up-regulated in almost all human tumors. It is known to have roles in the apoptosis (programmed cell-death) pathway, enhancing cell proliferation, and promoting angiogenesis, the development of new blood vessels $^{14,15}$. The use of antisense DNA in therapies targeted at reducing the expression of survivin may be effective in treating cancer. Recent results have shown that antisense DNA coupled to nanoparticles has an increased effectiveness compared to unconjugated DNA. 
The goal of this work was to develop a stable, highly effective SPION-based platform which can be targeted to specific cell types and serve as a vehicle for therapeutic drug delivery all the whole providing enhanced imaging contrast. The central hypothesis is that functionally modified SPIONs will preferentially target cancerous cells and thereby effectively delivering therapeutically significant concentrations of anti-tumor drugs. Three specific aims further explain the overall objective of this work: 1) identify design parameters for SPIONs that optimize aqueous stability and maximize amphiphilic character, 2) evaluate drug storage characteristics of SPIONs, and 3) demonstrate binding of antisense survivin DNA to SPIONs. Here, the amphiphilic shell coating the SPION core was functionalized with a targeting ligand. This targeting mechanism was then tested in A549 cancer cells, which overexpress the apoptosis inhibitory protein, Survivin. Targeting the Survivin mRNA sequence has been shown an effective antisense target suppresses survivin production and is used as the basis for SPION specificity. ${ }^{15}$ The antisense oligonucleotide sequence (aSL) used was: ${ }^{5} \mathrm{CCCAGCCTTCCAGCTCCTTG-}\left(\mathrm{CH}_{2}\right)_{6}{ }^{-}{ }^{3} \mathrm{NH}_{2}$. This oligonucleotide sequence was coupled to the biocompatible SPION and the reaction conditions for this process were optimized. Coupling of oligonucleotide to SPION is measured by spectroscopic techniques.

In this work, we focus on drug loading and molecular targeting for drug delivery. Epirubicin is a potent anticancer drug, but systemic dosing causes undesired side effects, including immune system suppression, since epirubicin affects 
healthy cells as well as cancer cells. ${ }^{16}$ Specific targeting of epirubicin release to cancer cells can increase the bioavailability of drug while reducing side effects to the patient. Here we report the loading of fluorescent anticancer drug Epirubicin (figure 3-1) onto modified SPIONs and the conjugation to tumor targeting antisense Survivin DNA.

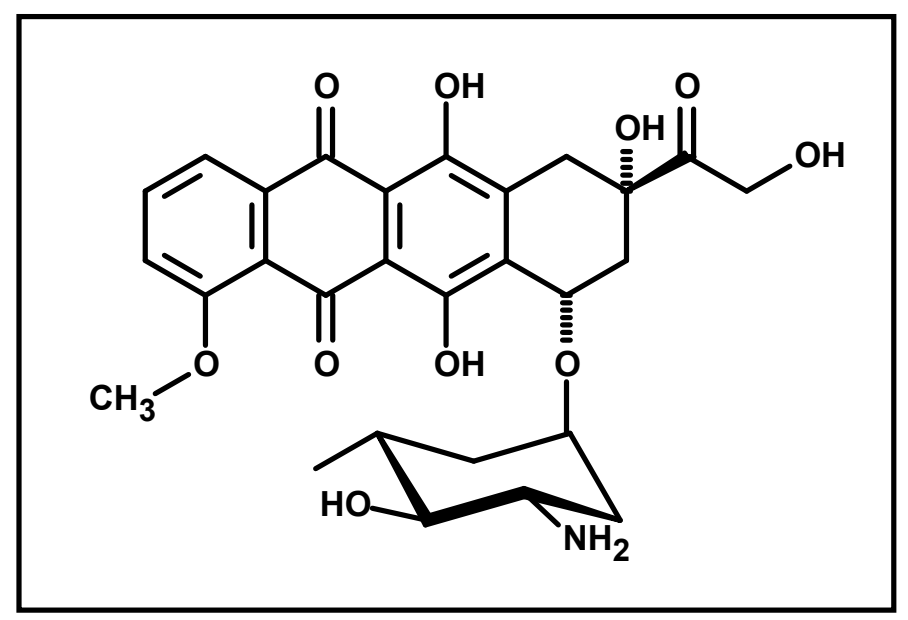

Figure 3-1: Chemical structure of Epirubicin 


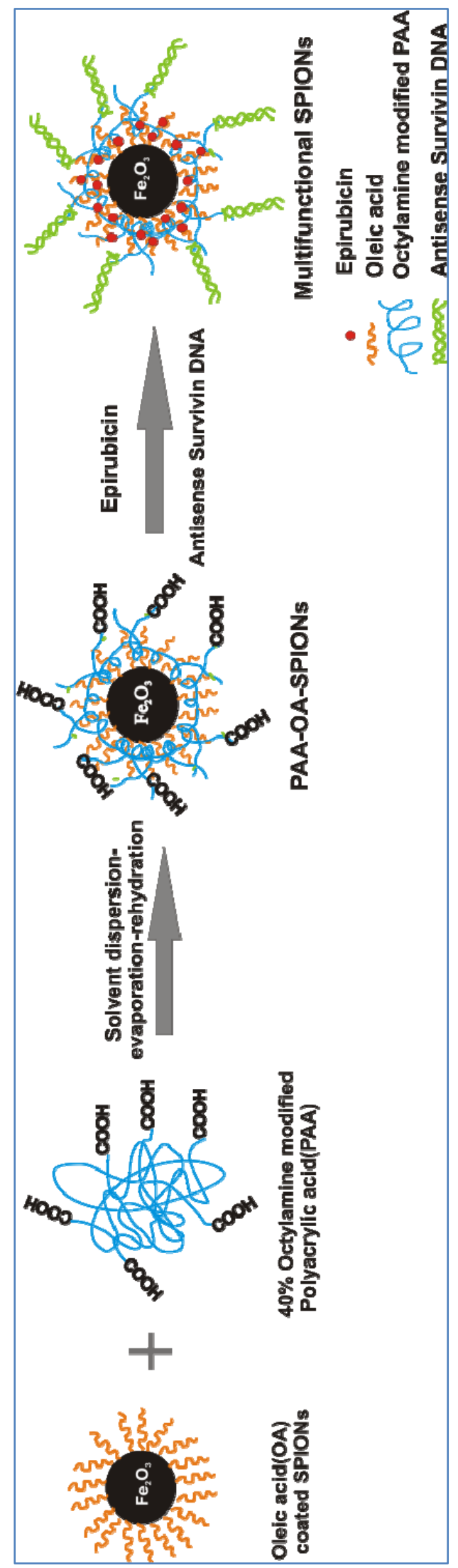

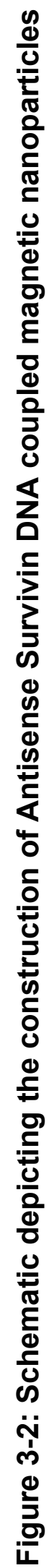




\subsection{Experimental}

\subsubsection{Materials and methods}

Epirubicin (EPI) in the form of the hydrochloride salt was purchased from SigmaAldrich. EPI base was prepared by mixing EPI with three equivalents of triethylamine (TEA) in dimethylformamide. Phosphate buffer saline (PBS) was 10 $\mathrm{mM}$ sodium monobasic phosphate, $2 \mathrm{mM}$ potassium dibasic phosphate containing $137 \mathrm{mM}$ sodium chloride and $2.7 \mathrm{mM}$ potassium chloride, $\mathrm{pH}$ 7.4. All chemicals including Iron pentacarbolyl, Oleic acid, Trimethylamine N-oxide were purchased from Sigma Aldrich (St.Louis, MO, USA). All the solvents which include DMF, triethylamine, toluene, hexane and ethanol are obtained from Fisher Scientific (Pittsburgh, PA, USA).

All reactions were conducted under an inert nitrogen atmosphere unless otherwise noted. For oligo synthesis: phosphoramidite DNA bases, fluorophore phosphoramidites and CPG columns were obtained from Glen Research (Sterling, VA). Oligonucleotides were prepared on an ABI 391 DNA synthesizer using standard coupling conditions except for the fluorophore phosphoramidite for which 3 min coupling time was used. The antisense survivin ligand (aSL) oligonucleotide was prepared without and with a fluorophore (f-aSL). In the case of $\mathrm{f}$-aSL, the fluorophore was located on the 5'-terminus and added during synthesis. The sequences of the oligonucleotides prepared were ${ }^{5} \mathrm{CCCAGCCTTCCAGCTCCTTG-}\left(\mathrm{CH}_{2}\right)_{6}-{ }^{3} \mathrm{NH}_{2}$ (aSL) and reverse complement 
${ }^{5}$ CAAGGAGCTGGAAGGCTGGG ${ }^{3}$ (aCS) with the 5'-trityl group removed the end of the synthesis.

\subsubsection{Synthesis and characterization of OA-SPIONs}

The oleic acid SPIONs (OA-SPIONs) were synthesized by a thermal decomposition method. The OA-SPIONs were well characterized by various analytical techniques such as transmission electron microscopy (TEM), dynamic light scattering (DLS), fourier transform infrared spectroscopy (FTIR) and thermo gravimetric analysis (TGA). The synthesis and characterization of OA-SPIONs are described in detail in Chapter 2. The same batch of SPIONs was used here in this work for surface modification using a different polymer with a functional group: $40 \%$ octylamine modified polyacrylic acid.

\subsubsection{Surface modification of SPIONs by modified polyacrylic acid}

Modified polyacrylic acid (PAA) has been previously used as a coating for the quantum dots for phase transfer and achieved a good yield. ${ }^{17,}{ }^{18}$ Briefly, 1800 molecular weight polyacrylic acid powder $(2.5 \mathrm{~g}, 1.39 \mathrm{mmol})$ was diluted in dry $\mathrm{N}, \mathrm{N}$-dimethylformamide $(35 \mathrm{~mL})$ and $\mathrm{EDC}-\mathrm{HCl}(2.66 \mathrm{~g}, 13.9 \mathrm{mmol})$ was dissolved. The solution was stirred for $30 \mathrm{~min}$ followed by the addition of octylamine $(2.3 \mathrm{~mL}, 13.9 \mathrm{mmol})$. The solution was stirred at room temperature overnight. Deionised water was added to precipitate the polymer which was 
isolated by centrifugation. Excess water was discarded. An aqueous solution of $1 \mathrm{M} \mathrm{NaOH}$ was added and solution was shaken overnight to resolubilize the polymer in water. Crude modified solution obtained was washed by ethyl acetate to improve the clarity of aqueous polymer suspension. The polymer was reprecipitated by adding dilute $\mathrm{HCl}$ to adjust the $\mathrm{pH}$ to 5 . Solid product was collected by centrifugation and dried under vacuum. The final product was carefully neutralized using $0.1 \mathrm{M} \mathrm{NaOH}$ solution and lyophilized for storage. This gives $40 \%$ octylamine modified PAA polymer which will be referred to as modified PAA copolymer.

OA-SPIONs were coated with the modified PAA copolymer by mixing solutions of each in miscible solvents (toluene and chloroform respectively). The solvents were evaporated by vacuum centrifugation and the PAA-OA-SPIONs were redissolved or resuspended in $\mathrm{DI}$ water at basic $\mathrm{pH} \sim 8$ by sonication for $2 \mathrm{hrs}$ at room temperature.

\subsubsection{Oligonucleotide Synthesis and purification}

Oligonucleotides were prepared on a $1 \mu \mathrm{mol}$ scale using an ABI-394 DNA and RNA Synthesizer using the standard coupling cycle and times. All oligonucleotides bore a 3' amino linker (6 carbon) so that they could be coupled to the SPIONs. The aSL oligonucleotide was prepare without and with a fluorophore (f-aSL), In the case of f-aSL, the fluorophore located on the 5'- 
terminus and added during synthesis. The sequences of the oligonucleotides prepared were ${ }^{5} \mathrm{CCCAGCCTTCCAGCTCCTTG-}\left(\mathrm{CH}_{2}\right)_{6}-{ }^{3} \mathrm{NH}_{2} \quad$ (aSL) and ${ }^{5}$ 'CAAGGAGCTGGAAGGCTGGG ${ }^{3}$ (aCS) with the 5'-trityl group removed at the end of the synthesis. Oligonucleotides were cleaved off the resin by treatment with concentrated $\mathrm{NH}_{4} \mathrm{OH}(28-30 \%, 1 \mathrm{ml})$ at room temperature for 1 hour and filtered through $0.2 \mu \mathrm{m}$ filter disk. The cleavage of protecting groups was accomplished by heating the filtrate at $55^{\circ} \mathrm{C}$ for $20 \mathrm{~h}$ and then dried down in a SpeedVac. Final purification of oligomers was achieved by (FPLC) using a BioRad TSK DEAE-5-PW column. Both oligonucleotides were purified using a linear gradient (10-70\% B over 60 min, Buffer A: $10 \mathrm{mM} \mathrm{NaOH,} \mathrm{pH} \mathrm{11.8,} \mathrm{Buffer} \mathrm{B:} 10$ $\mathrm{mM} \mathrm{NaOH}, 1 \mathrm{mM} \mathrm{NaCl}, \mathrm{pH}$ 11.8, flow rate $7 \mathrm{ml} / \mathrm{min}$, UV detection, $260 \mathrm{~nm}$ ). The oligomers were desalted with reverse phase Waters Sep-Pack (C-18) cartridges by $60 \% \mathrm{MeOH} /$ water elution.

\subsubsection{Coupling of antisense Survivin to SPIONs}

Conditions required for coupling of DNA were optimized. SPIONs (1 mg) in water were treated with EDC in solvent for $30 \mathrm{~min}$ and then an aqueous solution of the aSL oligonucleotide $(1.5 \mathrm{mg}$ ) was added and the mixture allowed to react for $3 \mathrm{~h}$ at $50^{\circ} \mathrm{C}$, with constant shaking. Excess DNA was removed from solution by filtration through a Vivaspin 100 kDa Molecular Weight Cut-off Filter (10 min, 250 $\mathrm{xg}$ ) washing three times with water. 


\subsection{Results \& Discussion}

In our work, to transform the hydrophobic oleic acid-SPIONs to water soluble nanoparticles for biological applications we used an octylamine polyacrylic acid co-polymer to assist in phase transfer process as well as a means for coupling the targeting biomolecules for targeted drug delivery.

An amphiphilic copolymer was prepared by coupling of octylamine to polyacrylic acid (PAA) backbone using EDC catalyzed coupling. Octylamine was coupled to approximately $40 \%$ of available carboxylic acid groups on PAA. This amphiphilic copolymer imparted aqueous stability to the SPIONs and provided coupling sites for attachment of targeting molecules. Copolymer structure was assessed by ATR-FTIR and dynamic light scattering.

\subsubsection{Fourier Transform Infrared Spectroscopy}

In figure $3-4$, the prominent peaks at $2750-3000 \mathrm{~cm}^{-1}$ correspond to symmetric/asymmetric stretching mode of the methyl/methylene units in both oleic acid and the PAA-copolymer. The sharp peak at $1710 \mathrm{~cm}^{-1}$ in OA-SPIONs is the $\mathrm{C}=\mathrm{O}$ stretch of oleic acid - the same region is broadened in the PAA-OASPIONs due to the increased number of acids on the PAA-copolymer. The presence of peak at $1630 \mathrm{~cm}^{-1}$ is attributed to the amide $\mathrm{C}=\mathrm{O}$ stretch from the 
octylamine-modified polymer. These peaks assignments are in accordance with literature assignments. ${ }^{19}$ 


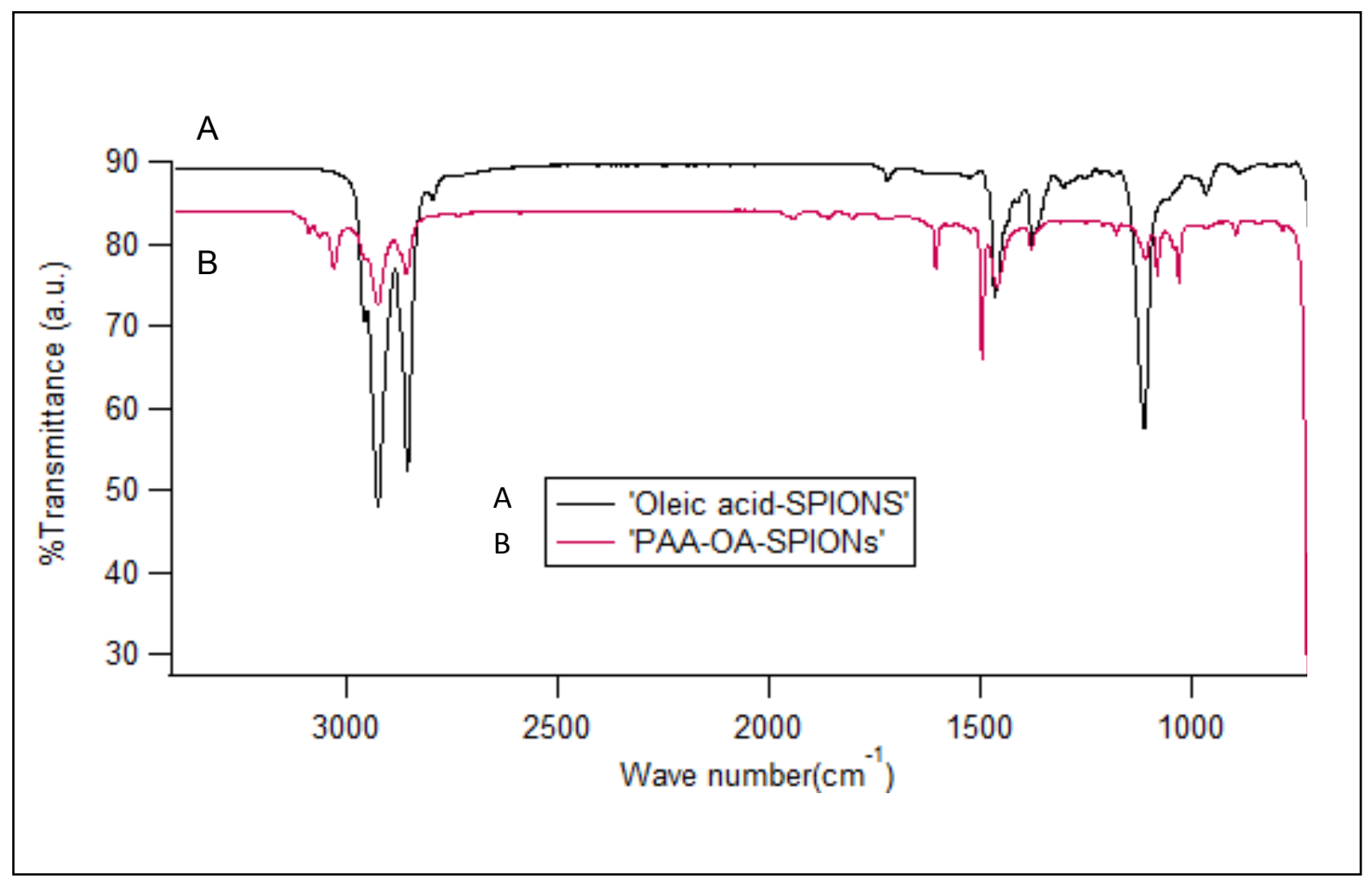

Figure 3-3: FTIR spectra of A) SPIONs (black trace) and B) modified PAA coated SPIONs (red trace) 


\subsubsection{Dynamic Light Scattering of PAA-OA-SPIONs}

The size and stability of modified PAA-OA-SPIONs in PBS $(\mathrm{pH} \sim 7.4)$ was studied by DLS and the average hydrodynamic radius found by intensity size distribution is $33 \mathrm{~nm}$. The zeta potential of these particles was found to be -82 $\mathrm{mV}$. At $\mathrm{pH} 7.4$, the carboxylic acid groups on the PAA-OA-SPION surface should be largely deprotonated, providing a high stabilizing surface charge. This is supported by the zeta potential measured.

\subsubsection{Loading of Epirubicin onto PAA-OA-SPIONs}

The Epirubicin (EPI) is a water-insoluble anticancer drug used for our study. A stock solution EPI $(0.5 \mathrm{mg} / \mathrm{mL})$ in DMF was used for loading and making standards. To the $3.6 \mathrm{~mL}$ of $1 \mathrm{mg} / \mathrm{mL}$ PAA coated SPIONs samples in water, $0.4 \mathrm{~mL}$ of $0.5 \mathrm{mg} / \mathrm{mL}$ EPI in DMF was added and stirred for $48 \mathrm{~h}$. During this time, the drug molecules partitions into the hydrophobic region of the surface modified SPIONs. After $48 \mathrm{~h}$, the drug-loaded PAA-SPIONs were separated from the drug solution using a strong magnet, supernatant drug solution was discarded and PAA-SPIONs were resuspended in deionised water. This process is repeated twice to ensure the removal of unadsorbed drug.

The amount of Epirubicin loaded into the SPIONs by the solvent diffusion method, after stirring it overnight was determined by using the drug absorbance 
in absorption spectroscopy at $485 \mathrm{~nm}$ wavelength. This first requires the preparation of a calibration curve and then measurement of the visible absorbance of the SPIONs in the absence and presence of Epirubicin (Figure 35). The ability of the SPIONs to entrap a drug is quantified by calculating the entrapment efficiency of the PAA coated SPIONs which is calculated using a calibration curve. This may be lesser than the actual loading efficiency of these systems since there might be change in molar absorptivity of dye on SPIONs.

\section{Calculations:}

Relative absorbance of EPI loaded SPIONs (obtained by subtracting absorbance of polymer-SPIONs from absorbance of EPI loaded polymer SPIONs) $=0.293$

EPI concentration loaded onto PAA-SPIONs (obtained from calibration plot) $=29 \mu \mathrm{g} / \mathrm{mL}$

Actual concentration of EPI used for loading $=50 \mu \mathrm{g} / \mathrm{mL}$

$\%$ loading efficiency (or) entrapment efficiency is given by:

(Drug loaded onto SPIONs / Total drug used for loading) *100

$\%$ loading efficiency $=(29 \mu \mathrm{g} / \mathrm{L} / 50 \mu \mathrm{g} / \mathrm{mL}) * 100=58 \%$ 


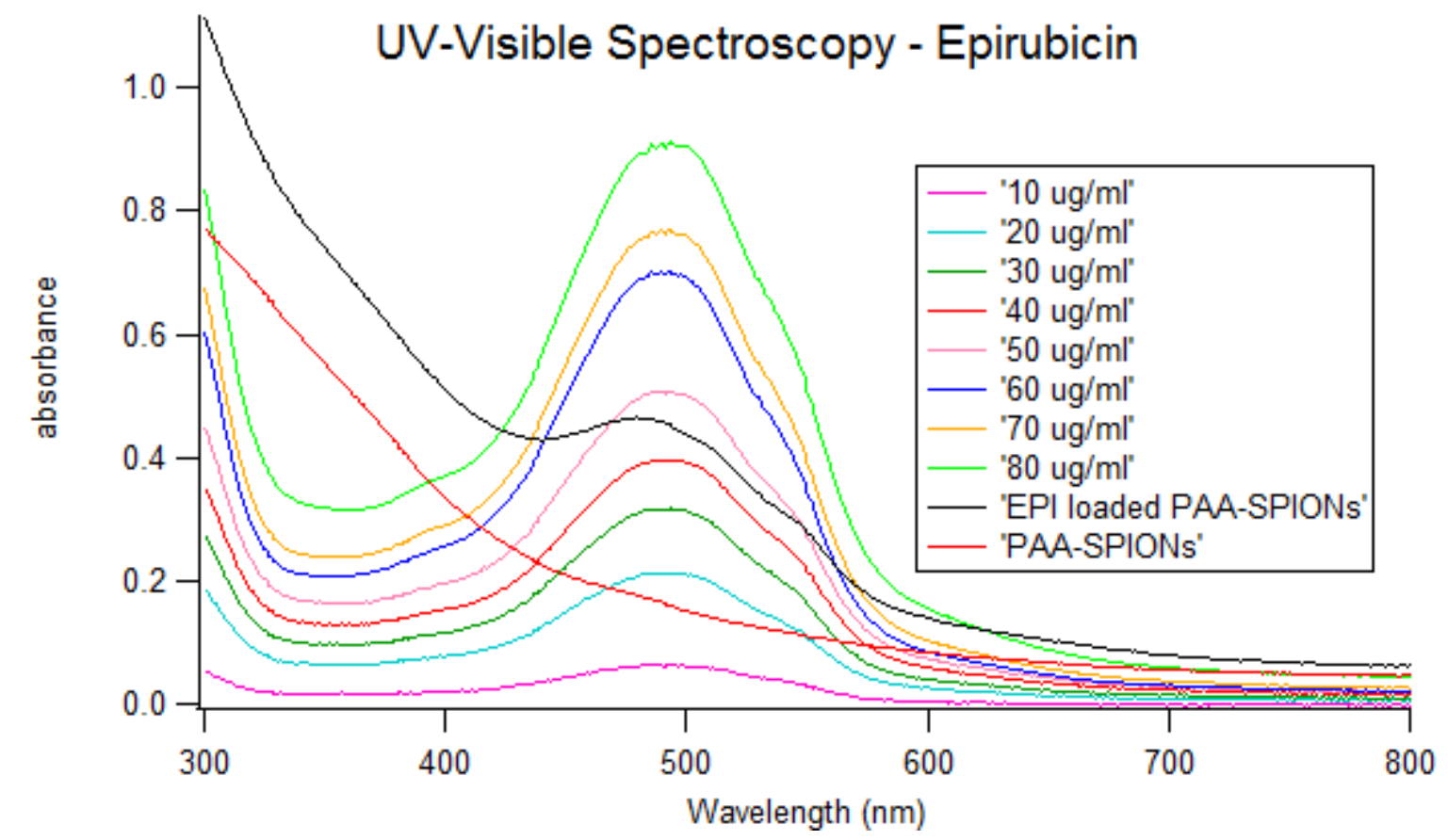

Figure 3-4. UV-Visible Spectroscopy of Epirubicin showing the spectra of standards ranging from $10 \mu \mathrm{g} / \mathrm{mL}$ to $80 \mu \mathrm{g} / \mathrm{mL}$ EPI with overlaid traces of EPI loaded PAA-OA-SPIONs and PAA-OA-SPIONs without drug. 


\subsubsection{Coupling of fluorescent antisense survivin ligand (f-aSL) to SPIONs}

Fluorescence was measured using a Shimadzu RF-5301PC spectrofluorophotometer in emission mode, with a scanning range from 300-600 $\mathrm{nm}$. Fluorometric analysis of $\mathrm{f}$-aSL alone and after coupling to the SPIONs was determined in water with $\lambda_{E x}=494 \mathrm{~nm}$ and $\lambda_{E m}=521 \mathrm{~nm}$. Figures 3-5 shows results measured by fluorescence studies of fluorescein modified antisense Survivin oligonucleotides in solution as well as when coupled to SPIONs. Table 3-1 shows the emission intensity of all the samples and the concentration of $f$ aSL on SPIONs calculated from the calibration curve. Coupling of survivin to SPIONs and its characterization was conducted by Dr. Gannett's group at health sciences. This data was obtained by Anand Narayanan from Dr. Gannett's group.

\begin{tabular}{|c|c|}
\hline $\begin{array}{c}\text { Concentration } \\
\text { (ng/ } \boldsymbol{\mu L} \text { ) }\end{array}$ & Emission @ 521nm \\
\hline SPION f-asL36 & 798 \\
\hline SPION f-asL 17 & 332 \\
\hline SPION f-asL 8.6 & 141 \\
\hline SPION f-asL 4.9 & 50 \\
\hline f-aSL 28 & 646 \\
\hline f-aSL14 & 212 \\
\hline f-aSL 7.0 & 85 \\
\hline f-aSL 1.4 & 9 \\
\hline
\end{tabular}

Table 3-1: Data obtained from fluorescence spectroscopy showing the concentration dependence of samples and their emission intensity at $521 \mathrm{~nm}$. 


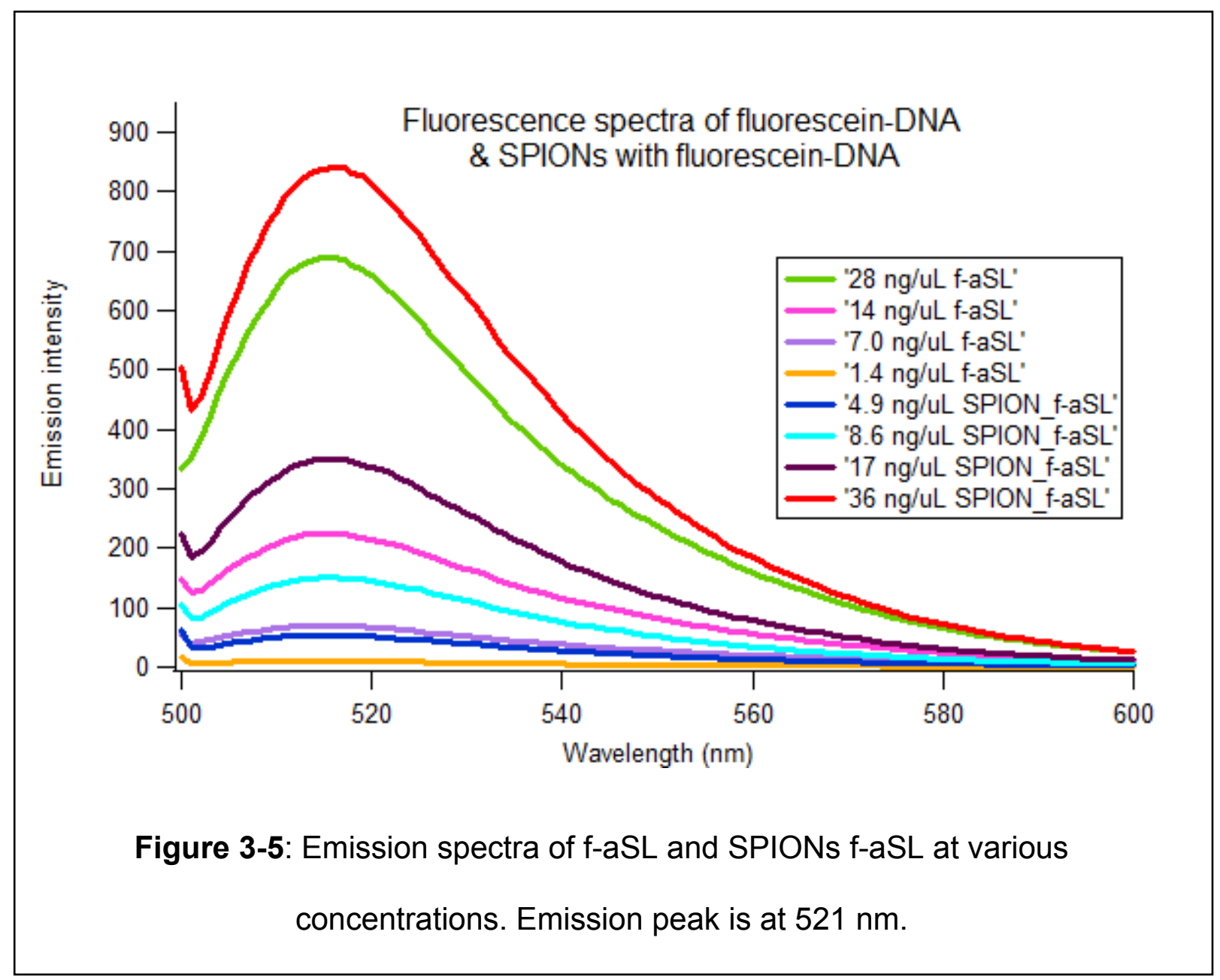

In this figure concentrations associated with SPIONs are calculated from the calibration curve of f-asL. The slope and intercept obtained from linear regression are $24 \pm 3(\mu \mathrm{L} / \mathrm{ng})$ and $70 \pm 47$.

Based on fluorescence data obtained, number of $f$-aSL per SPION was calculated assuming that the quantum yield remains the same for fluorescein even after tagging to DNA and attaching to SPION. Each SPION was expected to hold about $5 \mathrm{f}$-aSL molecules as per the calculated fluorescein molecules per SPION. 


\section{Calculations:}

\begin{tabular}{|l|r|l|}
\hline SPION density & 5.24 & $\mathrm{~g} / \mathrm{cm}^{\wedge} 3$ \\
\hline SPION radius & 5.01 & $\mathrm{~nm}$ \\
\hline Volume of SPION & 523 & $\mathrm{~nm} 3$ \\
\hline Mass of SPION & $2.74 \mathrm{E}-18$ & $\mathrm{~g} /$ particle \\
\hline $\begin{array}{l}\text { Mass of SPIONs in 10mg sample } \\
\text { of OA - SPIONs as per TGA }\end{array}$ & 0.00775 & $\mathrm{mg}$ \\
\hline \# of SPIONs & $2.82 \mathrm{E}+15$ & \\
\hline
\end{tabular}

\begin{tabular}{|c|c|c|c|c|c|c|}
\hline Sample & $\begin{array}{l}\text { Emission } \\
@ 521 \mathrm{~nm}\end{array}$ & Conc (ng/uL) & Molarity & moles & $\begin{array}{l}\text { molecules } \\
\text { of } \mathrm{f} \text {-aSL }\end{array}$ & $\begin{array}{l}\text { \# of } \mathrm{f} \text { - asL } \\
\text { molecules per np }\end{array}$ \\
\hline Calculation & & $\begin{array}{l}x= \\
(y+69.59) / 24.38\end{array}$ & $\begin{array}{l}\text { Conc }(\mathrm{g} / \mathrm{L}) / \\
332.31(\mathrm{~g} / \mathrm{mol})\end{array}$ & $M * V(L)$ & moles * A & $\begin{array}{l}\mathrm{f} \text { - asL molecules } \\
\text { / \# of SPIONs }\end{array}$ \\
\hline $\begin{array}{l}7.36 \mu \mathrm{g} \\
\text { SPION:faSL }\end{array}$ & 798 & 35.6 & 0.0001071 & $2.14 \mathrm{E}-08$ & $1.29 E+16$ & 4.30 \\
\hline $\begin{array}{l}3.68 \mu \mathrm{g} \\
\text { SPION:faSL }\end{array}$ & 332 & 16.5 & 4.955E-05 & 9.91E-09 & $5.97 E+15$ & 2.11 \\
\hline $\begin{array}{l}1.84 \mu \mathrm{g} \\
\text { SPION:faSL }\end{array}$ & 141 & 8.6 & 2.596E-05 & 5.19E-09 & $3.13 E+15$ & 1.11 \\
\hline $\begin{array}{l}0.92 \mu \mathrm{g} \\
\text { SPION:faSL }\end{array}$ & 50 & 4.9 & 1.473E-05 & 2.95E-09 & $1.77 E+15$ & 0.63 \\
\hline
\end{tabular}

Molecular weight of fluorescein $=332.31 \mathrm{~g} / \mathrm{mol}$

Avogadro number $(A)=6.023 * 10^{\wedge} 23$ 


\subsection{Conclusions \& future directions}

SPIONs were surface modified with a biocompatible polymer: octylamine modified polyacrylic acid. Aqueous solutions of surface modified SPIONs are amenable to moderate scale-up due to the simple synthesis. An anticancer drug Epirubicin is loaded efficiently and quantified by visible absorbance measurement. However, the bioavailability of the drug released from SPIONs should be further studied by in vitro drug release studies. The presence of functional groups on the polymer made the coupling of DNA possible. The coupling of antisense survivin DNA to PAA-OA-SPIONs using EDC-coupling successful and was verified by spectroscopic techniques. The coupling of oligonucleotide survivin to modified SPIONs will likely lead to active targeting of drug loaden SPIONs selectively to cancer cells. This targeting mechanism is being tested in A549 cancer cells, which over express Survivin. 


\section{References:}

1. Agasti, S. S.; Rana, S.; Park, M. H.; Kim, C. K.; You, C. C.; Rotello, V. M., Nanoparticles for detection and diagnosis. Advanced Drug Delivery Reviews 2010, 62, (3), 316-328.

2. Selvan, S. T.; Tan, T. T. Y.; Yi, D. K.; Jana, N. R., Functional and Multifunctional Nanoparticles for Bioimaging and Biosensing. Langmuir 2010, 26, (14), 11631-11641.

3. Neuberger, T.; Schopf, B.; Hofmann, H.; Hofmann, M.; von Rechenberg, B., Superparamagnetic nanoparticles for biomedical applications: Possibilities and limitations of a new drug delivery system. Journal of Magnetism and Magnetic Materials 2005, 293, (1), 483-496.

4. Pouliquen, D.; Lejeune, J. J.; Perdrisot, R.; Ermias, A.; Jallet, P., IronOxide Nanoparticles for Use as an Mri Contrast Agent - Pharmacokinetics and Metabolism. Magnetic Resonance Imaging 1991, 9, (3), 275-283.

5. Zhang, Y.; Zhou, D. J., Magnetic particle-based ultrasensitive biosensors for diagnostics. Expert Review of Molecular Diagnostics 2012, 12, (6), 565-571.

6. Brannon-Peppas, L.; Blanchette, J. O., Nanoparticle and targeted systems for cancer therapy. Advanced Drug Delivery Reviews 2004, 56, (11), 1649-1659.

7. Lu, A.-H.; Salabas, E. L.; Schüth, F., Magnetic Nanoparticles: Synthesis, Protection, Functionalization, and Application. Angewandte Chemie International Edition 2007, 46, (8), 1222-1244. 
8. Yallapu, M. M.; Othman, S. F.; Curtis, E. T.; Gupta, B. K.; Jaggi, M.; Chauhan, S. C., Multi-functional magnetic nanoparticles for magnetic resonance imaging and cancer therapy. Biomaterials 2010, 32, (7), 1890-1905.

9. Chouly, C.; Pouliquen, D.; Lucet, I.; Jeune, J. J.; Jallet, P., Development of superparamagnetic nanoparticles for MRI: Effect of particle size, charge and surface nature on biodistribution. Journal of Microencapsulation 1996, 13, (3), 245-255.

10. Veiseh, O.; Gunn, J. W.; Zhang, M. Q., Design and fabrication of magnetic nanoparticles for targeted drug delivery and imaging. Advanced Drug Delivery Reviews 2010, 62, (3), 284-304.

11. Li, S. D.; Chen, Y. C.; Hackett, M. J.; Huang, L., Tumor-targeted delivery of siRNA by self-assembled nanoparticles. Molecular Therapy 2008, 16, (1), 163169.

12. Jansen, B.; Zangemeister-Wittke, U., Antisense therapy for cancer - the time of truth. Lancet Oncology 2002, 3, (11), 672-683.

13. Fulda, S., Inhibitor of Apoptosis (IAP) proteins as therapeutic targets for radiosensitization of human cancers. Cancer Treatment Reviews 1996, 38, (6), 760-766.

14. Medarova, Z.; Pham, W.; Farrar, C.; Petkova, V.; Moore, A., In vivo imaging of siRNA delivery and silencing in tumors. Nat Med 2007, 13, (3), 372377. 
15. Mita, A. C.; Mita, M. M.; Nawrocki, S. T.; Giles, F. J., Survivin: Key Regulator of Mitosis and Apoptosis and Novel Target for Cancer Therapeutics. Clinical Cancer Research 2008, 14, (16), 5000-5005.

16. Swami, U.; Chaudhary, I.; Ghalib, M. H.; Goel, S., Eribulin-A review of preclinical and clinical studies. Critical Reviews in Oncology Hematology 2012, 81, (2), 163-184.

17. Shen, H. Y.; Jawaid, A. M.; Snee, P. T., Poly(ethylene glycol) Carbodiimide Coupling Reagents for the Biological and Chemical Functionalization of Water-Soluble Nanoparticles. Acs Nano 2009, 3, (4), 915923.

18. Sato, K.; Tachibana, Y.; Hattori, S.; Chiba, T.; Kuwabata, S., Polyacrylic acid coating of highly luminescent CdS nanocrystals for biological labeling applications. Journal of Colloid and Interface Science 2008, 324, (1-2), 257-260.

19. Santra, S.; Kaittanis, C.; Grimm, J.; Perez, J. M., Drug/Dye-Loaded, Multifunctional Iron Oxide Nanoparticles for Combined Targeted Cancer Therapy and Dual Optical/Magnetic Resonance Imaging. Small 2009, 5, (16), 1862-1868. 


\section{Chapter 4}

Superparamagnetic Iron Oxide Nanoparticles (SPIONs) as a support for analytical sensing 


\subsection{Introduction}

Nanoparticles are widely being used in various fields such as drug delivery, imaging, labeling, sensing, analytical and bio-separations. ${ }^{1-3}$ Surface modification of nanoparticles facilitates conjugation of biomolecules..$^{4-6}$ Recently, nanoparticles are being conjugated to aptamers. ${ }^{7}$ In chapter 2 and 3,1 used surface modified magnetic nanoparticles for biological applications. Here in this chapter, I will demonstrate the use of magnetic nanoparticles for analytical applications. SPIONs are used for immobilizing aptamers via biotin-avidin chemistry and used as capture tools for steroids in the present work.

Aptamers (also known as molecular recognition elements) are developed by an in vitro selection process called SELEX. ${ }^{8}$ SELEX (Systematic Evolution of Ligands by Exponential Enrichment) is a process wherein the DNA/RNA ligands are generated from a random pool of modified or unmodified DNA or RNA. The ligands selected through this process are called aptamers (single stranded synthetic DNA or RNA capable of folding into unique 3D structures). Aptamers are widely being used as molecular recognition elements in the fields of biosensing, diagnostics and therapeutics. ${ }^{9-13}$ Previously, antibodies were coupled to nanoparticles for using them in bio-applications. ${ }^{14,}{ }^{15}$ However, aptamers posses additional advantages such as low molecular weights, easy and reproducible synthesis, low toxicity and high binding affinity and selectivity. The 
ability of aptamers to distinguish targets from subtle structural differences and bind any target with high affinity made them unique tools in bio-analytical applications. ${ }^{9,16}$

There is an increasing use of aptamers in biosensors for the detection of small molecules. ${ }^{17-19} \mathrm{Kim}$ et al. discovered a 76 mer DNA aptamer selective to $17 \beta$ estradiol $(E 2) .{ }^{20}$ The chemical structure of $17 \beta$-estradiol is shown in figure 4.1. This aptamer has been proved to have high affinity to estradiol with a dissociation constant $\left(\mathrm{K}_{\mathrm{d}}\right)$ of $0.13 \mu \mathrm{M}$ by electrochemical method. Gioi et al. used aptamer anchored glass microbeads to preconcentrate estradiol in spiked water samples and separate bound and unbound fractions using HPLC. ${ }^{21}$ They reported a dissociation constant of $35 \mu \mathrm{M}$.

In our group, we used 76mer-DNA aptamer to assess its binding affinity with estradiol using stacking enhanced capillary electrophoresis previously developed for steroids separation. ${ }^{22}$ We used streptavidin coated magnetic micro beads as the capture elements to separate the bound estradiol from the unbound fraction. In the present work, streptavidin coated magnetic nanoparticles are used as the capture elements and the separation of bound fraction from the unbound fraction of estradiol is carried out in a micro capillary. Specific binding conditions (solvent, ionic strength, $\mathrm{pH}$ ) used is similar to previous work. ${ }^{23}$ Samples are analyzed on capillary electrophoresis $(\mathrm{CE})$ to quantify the bound fraction of estradiol. 
Capillary electrophoresis is an effective microscale separation method. UVvisible absorbance is most commonly used for detection but generally has poor limit of detection. Stacking and sweeping techniques are used to improve the detection limits. ${ }^{24}$ In the present work, $\mathrm{pH}$-mediated stacking method is used for quantification of estradiol captured by the aptamer.

Use of magnetic nanoparticles and in-capillary separation under strong magnetic field helps lay out a platform for developing nanoscale devices for detection of small molecules.

Figure 4-1: Chemical structure of 17ß-estradiol (E2)<smiles>C[C@]12CC[C@H]3c4ccc(O)cc4CC[C@H]3[C@@H]1CCC2O</smiles> 


\subsubsection{Theory of Capillary electrophoresis}

Electrophoresis is migration of ions in solution under the influence of electric field. When a charged molecule $(q)$ is placed in a constant electric field $E$, it experiences an accelerating force $\mathrm{qE}$ and a retarding frictional force fu, where $u$ is velocity of the ion and $f$ is friction coefficient.

(Accelerated force) $q E=\mathrm{fu}$ (frictional force) Equation 4.1

$\mathrm{U}=\mathrm{q} / \mathrm{f} \mathrm{E}=\mu_{\mathrm{ep}} \mathrm{E}$ .Equation 4.2

Where $\mu_{\mathrm{ep}}$ is electrophoretic mobility. It is a proportionality constant between velocity of ion and electric field strength.

Figure 4-2: Charged molecule placed in a constant electric field experiencing different types of forces

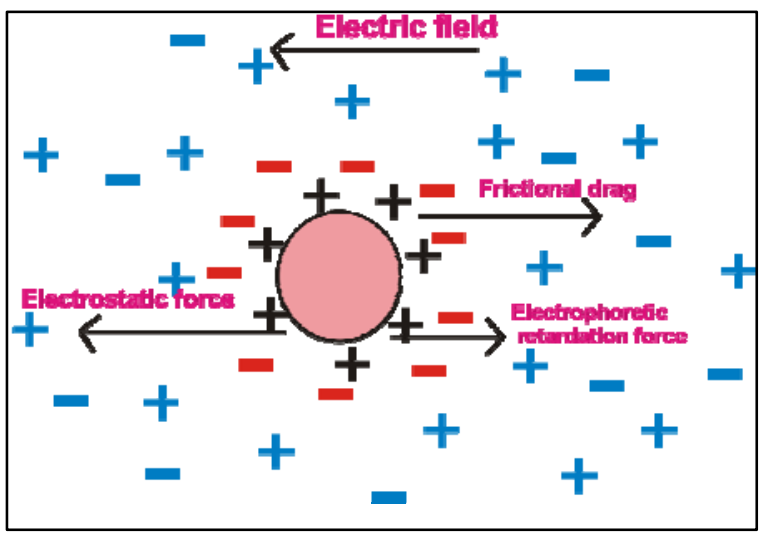


In capillary electrophoresis $(\mathrm{CE})$, sample is injected into a fused silica $\left(\mathrm{SiO}_{2}\right)$ capillary filled with running buffer and high voltage is applied to separate the components. All the solutes migrate with different mobilities and separation occur based on size to charge ratio. Electrophoretic mobility and electro-osmotic flow dictates the separation in CE. A block diagram of capillary electrophoresis is shown in figure 4-3.

Figure 4-3: Block diagram of capillary electrophoresis

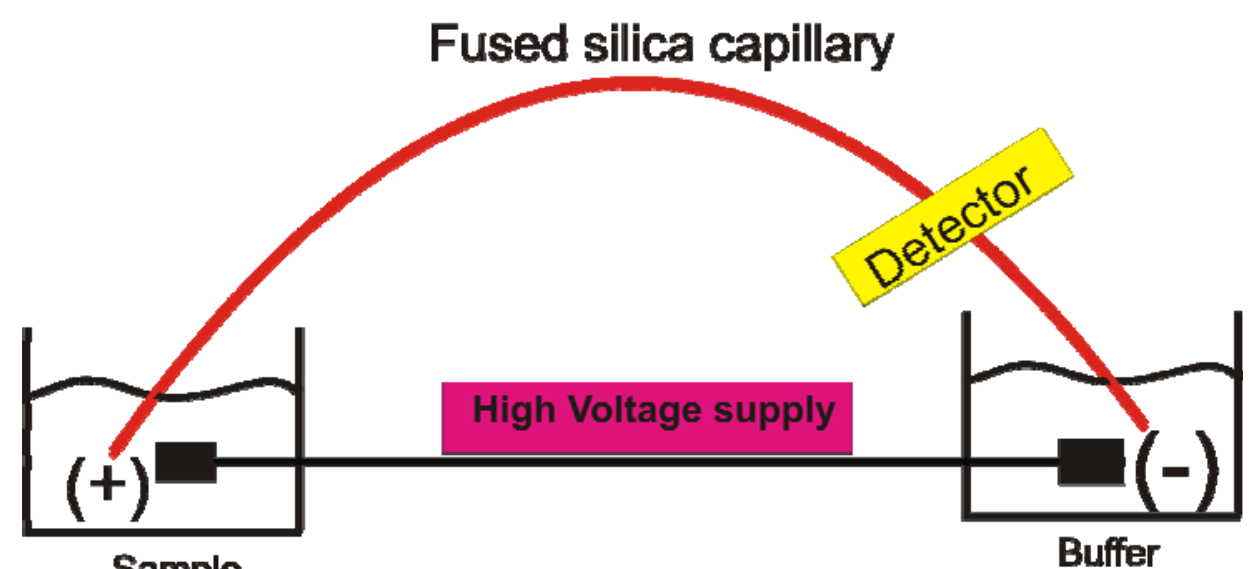

Sample

Buffer 
At $\mathrm{pH}$ above $\sim 2.5$, the silanol groups in the capillary are deprotonated and posses negative charge. This attracts the cations in the solution to the negative surface to partially neutralize the charge. This forms an electric double layer at the capillary wall. Electroosmotic flow ( $\left.\mu_{o s}\right)$ is a uniform plug like flow of solute molecules towards cathode driven by the cations in diffuse double layer in the capillary. A pictorial representation of electro-osmotic flow is shown in figure 4-4.

Figure 4-4: Representation of Electro-osmotic flow

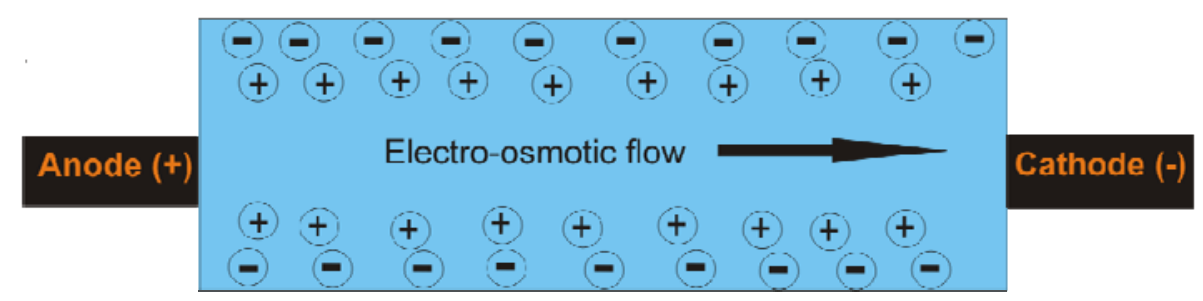

Electrophoretic mobility $\left(\mu_{\mathrm{ep}}\right)$ is the migration of charged ions under the influence of applied electric field. It is directly proportional to the charge on the ion (q) and inversely proportional to the frictional coefficient (f). A pictorial representation of electrophoretic mobility is shown in figure 4-5. The apparent or observed mobility 
of the analytes is the sum of electro-osmotic flow and electrophoretic mobility given by equation 4.3 .

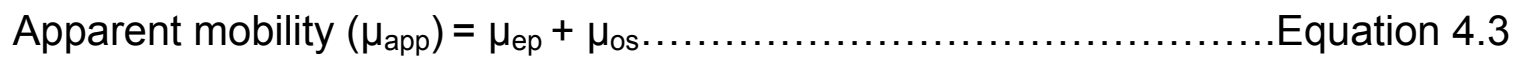

The movement of analytes due to applied voltage from anode to cathode is normal polarity (shown in figure 4-5). The opposite setting is referred to as reverse polarity.

Figure 4-5: Representation of Electrophoretic mobility

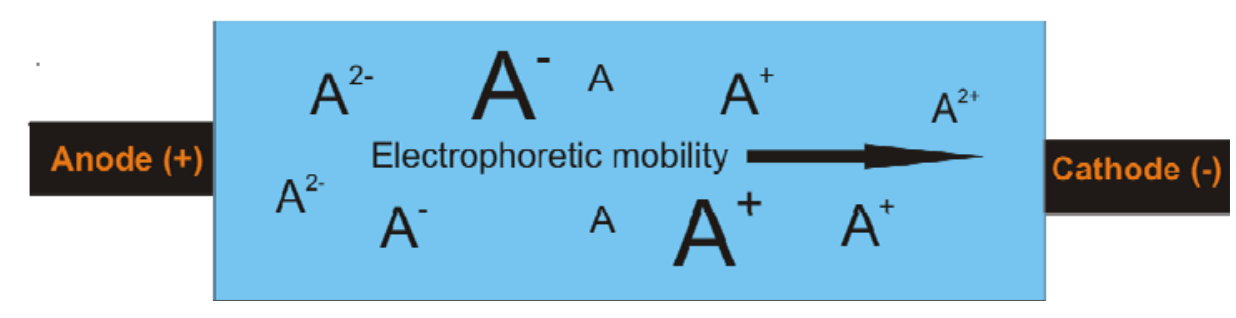

\subsubsection{Advantages of CE}

Capillary electrophoresis is a well known separation technique which offers several advantages. ${ }^{25,26}$ There is no stationary phase in CE and the flow of 
sample in the open capillary tube is a plug flow as opposed to hydrodynamic flow in a chromatographic column. This eliminates the longitudinal diffusion and mass transfer mechanisms involved in the chromatographic separations. Hence, CE provides high efficiency and resolution. Since CE uses micron size capillaries, it requires extremely small sample volumes (as low as microliters to nanoliters). The processing time of samples is faster than other separation techniques like chromatography. CE can be used for separation and analysis of a wide range of analytes both charged as well as neutral. It is especially a useful tool for analyzing biomolecules such as proteins and nucleic acids. ${ }^{27-29}$

\subsubsection{Micellar electrokinetic chromatography (MEKC)}

Micellar electrokinetic chromatography (MEKC) is a type of CE which includes a pseudo stationary phase of micelles. It offers many advantages over capillary zone electrophoresis. ${ }^{30}$ MEKC separates neutral molecules and ions. Surface active agents or surfactants form micelles above their critical micellar concentrations. Surfactants can be anionic, cationic, zwitterionic and neutral. Any of these types or a combination can be used as pseudo stationary phase in MEKC. Sodium dodecyl sulfate (SDS), an anionic surfactant, is extensively used in MEKC. The electrophoretic mobility of negatively charged micelles is from cathode to anode while the electro-osmotic flow is from anode to cathode. The apparent mobility of the micelles is towards cathode as EOF is dominant. The migration time of neutral analytes depends on their partitioning between the 
micelles. Cyclodextrins (CD) are neutral, inert and stable cyclic oligomers of $\alpha-D-$ glucose with a hollow cavity. They are widely used for separation of optical isomers and enantiomers. ${ }^{31-33}$ The interior of cyclodextrin is non-polar and hence accommodates hydrophobic neutral analytes while the exterior is polar making it soluble in water. Cyclodextrins move along with EOF as they are neutral. These molecules have low UV absorptivity at short wavelengths.

Figure 4-6: Strutures of sodium dodecyl sulfate and cyclodextrin<smiles>CCCCCCCCCCCCO[AsH3]</smiles>

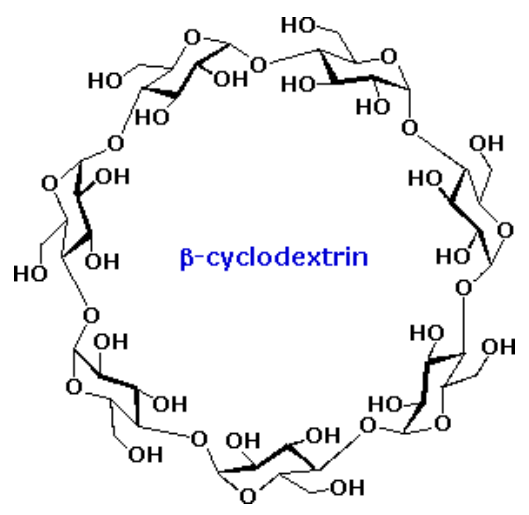




\subsubsection{Instrumentation}

The basic instrumentation of capillary electrophoresis includes a micron size fused silica capillary with the two ends placed in buffer reservoirs containing platinum electrodes. The sample is injected into the capillary either hydrodynamically by applying pressure or electrokinetically by applying voltage on injection side. In normal polarity, sample is injected from anode to cathode. In reverse polarity, sample is injected from cathode to anode. The detector is placed on the outlet side of the capillary. Most of the instruments commonly have UV-Visible detector. However, fluorescence and electrochemical detectors can also be used on CE. The photographs of CE instrumentation are shown in figure 4-7. The plot obtained from the detector response is known as electropherogram. 
Figure 4-7. Photographs of the Beckman $®$ automated capillary electrophoresis machine used in the work. The light source is a UV-visible absorbance, with a PMT or PDA detector.
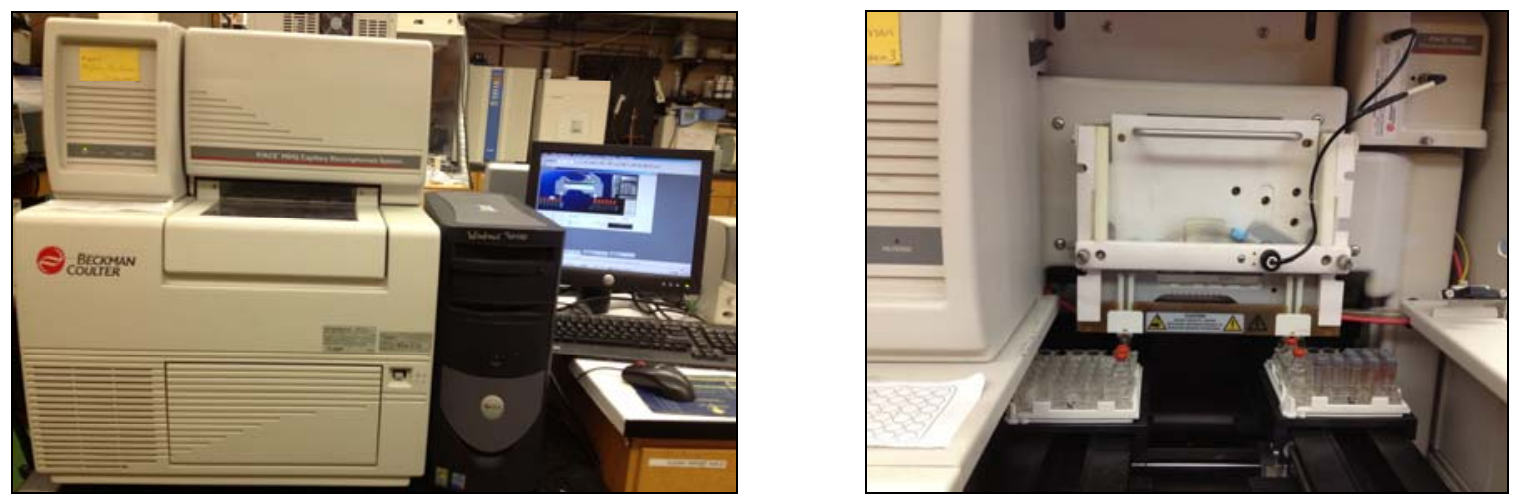


\subsection{Materials and methods}

\subsubsection{Reagents and materials}

The biotinylated DNA aptamer was synthesized by IDT (Coralville, IO, USA). Sodium hydroxide, sodium chloride, potassium chloride, sodium dodecyl sulfate (SDS), carboxymethyl- $\beta$-cyclodextrin, testosterone, and 3-[cyclohexylamino]-1propanesulfonic acid (CAPS) were purchased from Sigma-Aldrich (St. Louis, MO, USA). Streptavidin modified superparamagnetic iron oxide nanoparticles (SHS30) were purchased from Ocean Nanotech (Springdale, AR, USA). Methanol was purchased from EMD Biosciences (La Jolla, CA, USA). 17ßestradiol and estrone were purchased from Steraloids, Inc. (Newport, RI, USA). 17a-ethynyl estradiol was purchased from Cayman Chemical (Ann Arbor, MI, USA). Hydroxypropyl- $\beta$-cyclodextrin (HPCD) was purchased from VWR (West Chester, PA, USA). Hydrochloric acid, magnesium chloride, and sodium phosphate monobasic salt were purchased from Fisher Scientific (Fair Lawn, NJ, USA). Tris hydrochloride was purchased from Mallinckrodt Maker Inc. (Phillipsburg, NJ, USA). Neodymium block magnet BX8X8X8-N52 (1 1/2" x 1 1/2" x 1 1/2" thick) used for separation of SPIONs was purchased from $K$ \& $J$ magnetics, inc. (Jamison, PA, USA). Deionized water was obtained from an Elga Purelab ultra water system (Lowell, MA).

\subsubsection{Instrumentation}

Separations were performed using a Beckman Coulter P/ACE MDQ capillary electrophoresis instrument (Beckman Coulter, Fullerton, CA, USA) shown in 
Figure 4-7. The P/ACE MDQ was equipped with a photodiode array (PDA) for UV-visible absorbance detection which provided simultaneous determination of steroids at multiple wavelengths of $200,225,254 \mathrm{~nm}$. An Oven was used in sample preparation for incubation for biotin-streptavidin release. Centrifugation was performed on a picofuge (Stratagene, La Jolla, CA, USA). A vacuum centrifuge (Savant, NY, USA) is used for the drying of $10 \%$ ethanol solution in the sample preparation. The Thermo Scientific Nanodrop 1000 is used to determine DNA concentration in the samples.

\subsubsection{DNA aptamer}

The DNA aptamer (GCT TCC AGC TTA TTG AAT TAC ACG CAG AGG GTA GCG GCT CTG CGC ATT CAA TTG CTG CGC GCT GAA GCG CGG AAG C) was synthesized with a biotin connected to the 5' base via a 15 atom spacer arm (tetra-ethyleneglycol). The aptamer was reconstituted at a concentration of 10 $\mu \mathrm{M}$ in a binding buffer similar to that previously reported $(200 \mathrm{mM} \mathrm{NaCl}, 10 \mathrm{mM}$ $\mathrm{MgCl}_{2}, 25 \mathrm{mM}, \mathrm{KCl}, 5 \%$ ethanol, $100 \mathrm{mM}$ Tris- $\mathrm{HCl}$ buffered at $\left.\mathrm{pH} 8\right) .{ }^{20}$

\subsubsection{Capillary Electrophoresis separation conditions}

$30 \mathrm{~cm}$ long fused-silica capillary with an inner diameter of 25 um and outer diameter of 360 um (Polymicro Technologies, LLC, Phoenix, AZ, USA) is used for separations. The detection window was placed at $10.2 \mathrm{~cm}$ from the site of injection. The capillary is maintained at $25^{\circ} \mathrm{C}$ during all the flushes and runs. The separation buffer used comprised of $30 \mathrm{mM}$ SDS, $13 \mathrm{mM}$ hydroxypropyl- $\beta$ - 
cyclodextrin, $200 \mathrm{mM}$ phosphate buffered at $\mathrm{pH} 2.5$. Reverse polarity at $16 \mathrm{kV}$ is used for separation (cathode at the point of injection and anode at the outlet).

\subsubsection{Binding experiment}

The biotinylated aptamer is coupled to $30 \mathrm{~nm}$ size streptavidin coated SPIONs. In this experiment, $50 \mu \mathrm{L}$ of SPIONs with a concentration of $1 \mathrm{mg} / \mathrm{mL}$ is taken. An approximate binding capacity of 850 picomoles of single-stranded oligonucleotide per mg nanoparticles was reported by the manufacturer. Approximately $50 \mu \mathrm{L}$ of SPIONs can bind to 40 picomoles of biotinylated DNA. $50 \mu \mathrm{L}$ SPIONs was passed through magnet and reconstituted in $20 \mu \mathrm{L}$ of binding buffer. Therefore, the DNA binding concentration of SPIONs is $2 \mu \mathrm{M}$ (40 picomoles).

The aptamer is denatured at $90{ }^{\circ} \mathrm{C}$ for 10 mins in binding buffer and cooled to room temperature (shown in figure 4-8). The estradiol is added to denatured DNA at the specified concentration and incubated for $1 \mathrm{hr}$ at $25^{\circ} \mathrm{C}$. A sample of SPIONs is taken into a microcapillary by capillary action and held in a high magnetic field (on the edge of a strong magnet) for about 20 min. The glass microcapillary used in this procedure can hold $100 \mu \mathrm{L}$ volumes. It is approximately $11.5 \mathrm{~cm}$ long and $340 \mu \mathrm{m}$ in diameter. During the waiting period SPIONs settle down on the sidewall and the supernatant is eluted using a rubber bulb with a 10-100 $\mu \mathrm{L}$ plastic pipette tip. Low quality SPIONs having differences in size and magnetic nature are discarded in the supernatant. SPIONs were then reconstituted deionised water. Later the aptamer-estradiol complex is added to 
washed SPIONs and incubated for 30 mins. After incubation, solution is filled in a microcapillary and held in a high magnetic field about $20 \mathrm{~min}$. The unbound DNA and estradiol is washed off the SPIONs by reconstituting with binding buffer, placing on the magnet and eluting the supernatant. This removes any residual DNA or estradiol. The washing procedure is shown in figure 4-9. The washed SPIONs are reconstituted in $10 \%$ ethanol. To release the DNA bound to the SPIONs, they are heated at $70{ }^{\circ} \mathrm{C}$ for $10 \mathrm{~min}$. Heating at this temperature reportedly dissociates biotin-streptavidin interaction. ${ }^{34}$ The solution is cooled to room temperature and pulled into micro capillary which is placed on the edge of the magnet. The SPIONs are held in place by the magnet; the solution is eluted into a vial, and dried in a vacuum centrifuge. The dried estradiol is then reconstituted by stack buffer comprised of $1 \mathrm{mM}$ carboxymethyl- $\beta$-cyclodextrin and $100 \mathrm{mM}$ CAPS buffered at $\mathrm{pH}$ 10. The sample is then injected into separation capillary using the sample stacking method previously reported..$^{22}$ This stacking mechanism is proved to increase the steroid concentration by 10 fold. The schematic of the experimental procedure is represented in figure 4-10.

\subsubsection{Separation mechanism}

The running buffer filled in the capillary is $200 \mathrm{mM}$ phosphate buffer at pH 2.5 comprised of $30 \mathrm{mM}$ SDS and $13 \mathrm{mM}$ HPCD. The stacking buffer used is $1 \mathrm{mM}$ CMCD in 50 mM CAPS buffered at $\mathrm{pH} 10$ with $5 \%$ methanol. Methanol aids in solubilization of hydrophobic steroids. The estradiol steroid is electrokinetically injected into the capillary by applying $10 \mathrm{kV}$ high voltage at reversed polarity for 
$60 \mathrm{sec}$. At high $\mathrm{pH}$ of the stacking buffer, CMCDs are deprotonated and carry the neutral steroids. As the sample in stacking buffer is injected, it forms a $\mathrm{pH}$ junction in the capillary concentrating the neutral steroid-CMCD complex. Electro-osmotic flow is insignificant in the capillary which slows down the movement of neutral molecules such as HPCD. Electrophoretic mobility helps in the movement of negatively charged SDS molecules. The steroids partition between negatively charged SDS and CMCDs and are separated at $16 \mathrm{kV}$ under reverse polarity. A schematic of separation mechanism is shown in figure 4-11. 
Figure 4-8: Denaturing of DNA aptamer and its renaturing at room temperature followed by incubation with estradiol and SPIONs

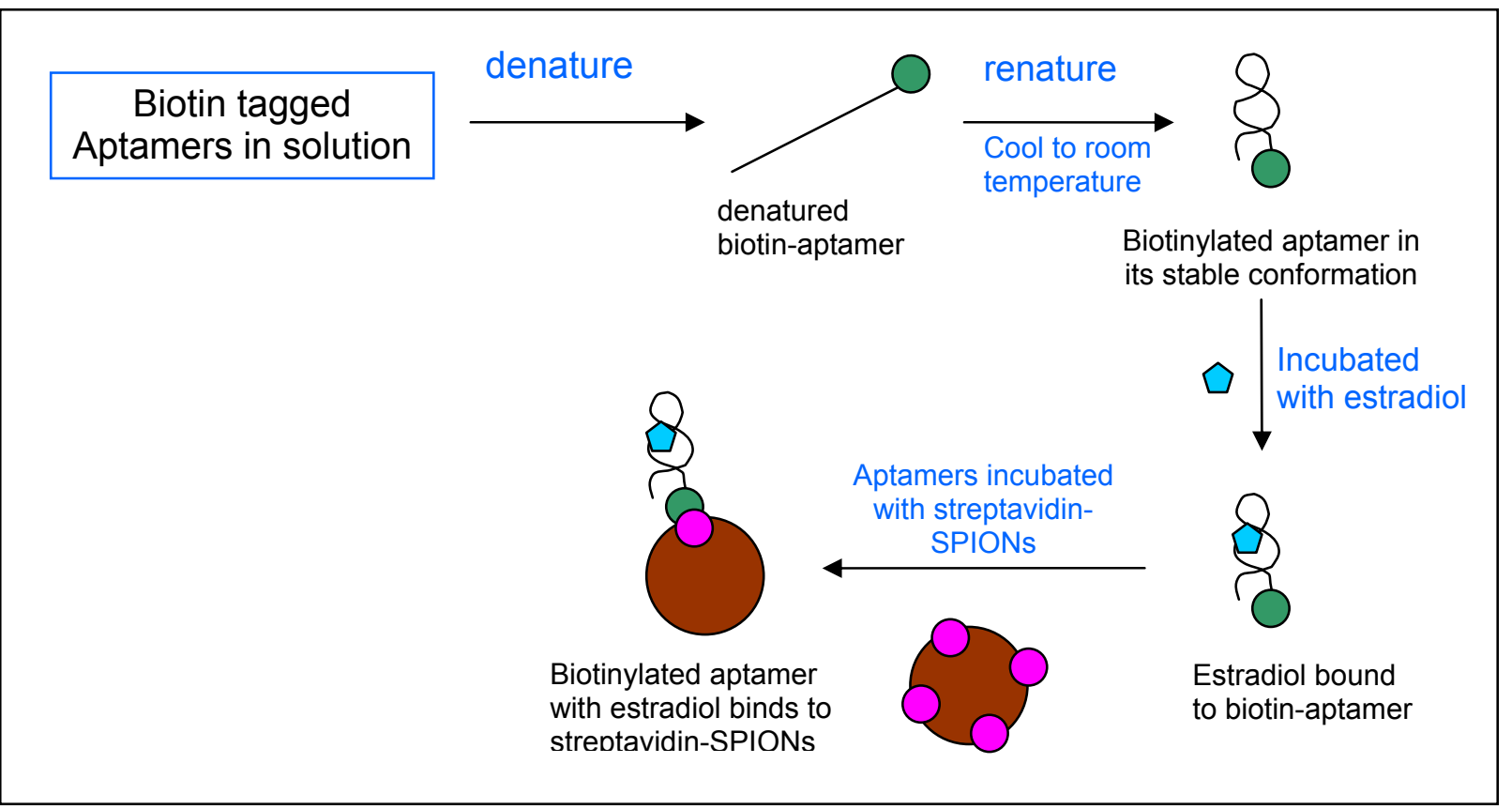

Figure 4-9: Schematic of SPIONs washing procedure

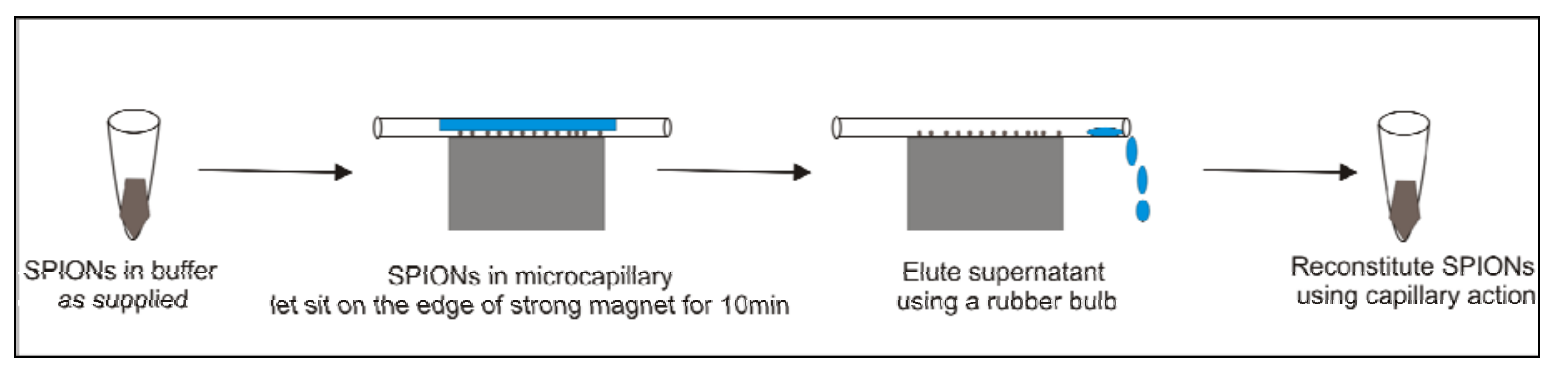


Figure 4-10: Schematic of binding affinity study of 76mer with estradiol using

\section{SPIONs as capture elements}

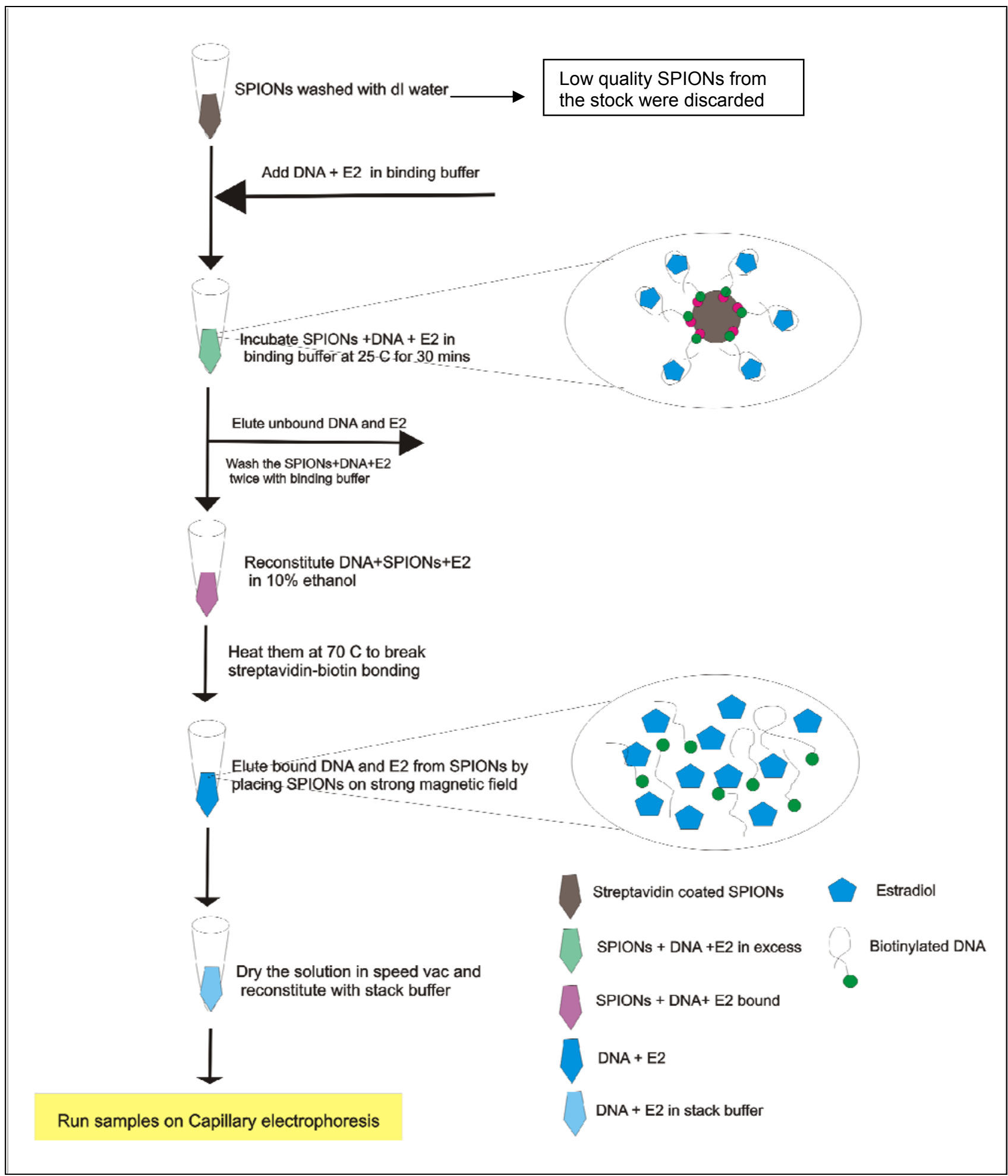


Figure 4-11: Schematic of $\mathrm{pH}$ mediated micellar electrokinetic chromatography (MEKC) stacking of estradiol. Separation buffer used was $\mathrm{pH} 2.5$ phosphate buffer containing sodium dodecyl sulfate (SDS) and Hydroxypropyl cyclodextrin (HPCD). Stack buffer used is pH 10 CAPS buffer containing Carboxymethyl cyclodextrins (CMCD)

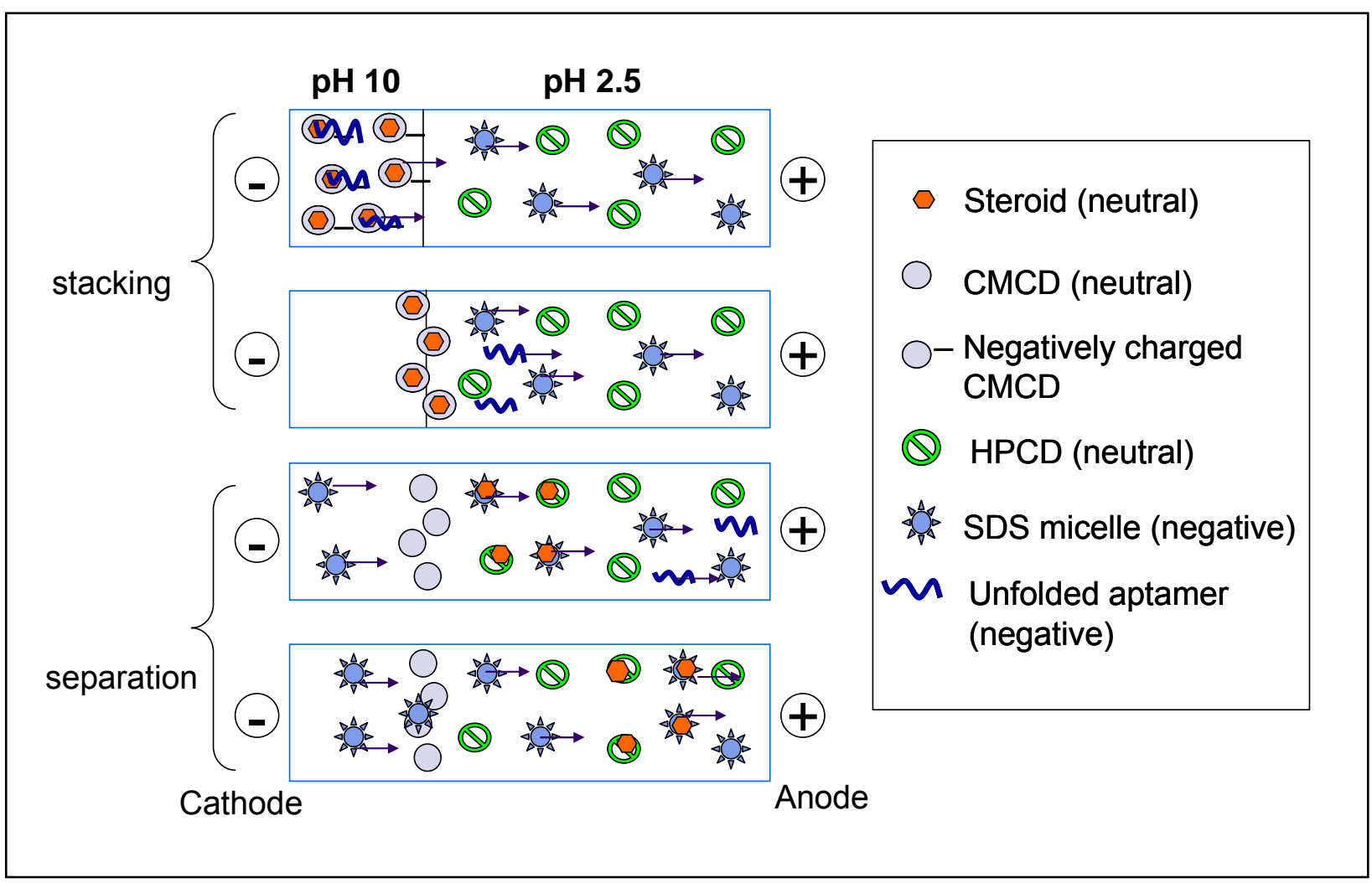




\subsection{Results and discussion}

\subsubsection{Aptamer binding}

Aptamer immobilized on SPIONs is incubated with concentrations of estradiol ranging from $18 \mu \mathrm{M}$ to $95 \mu \mathrm{M}$ to determine the binding affinity. The unbound estradiol is removed by washing with the binding buffer. Binding buffer is used to maintain the right confirmation of aptamer. The estradiol bound to the aptamer on SPIONs is released into $10 \%$ ethanol by heating at $70{ }^{\circ} \mathrm{C}$. This solution is dried in the vacuum centrifuge. It is then reconstituted in stack buffer and quantified by sample stacking and capillary electrophoresis. This process of drying and reconstituting ensures the volumes of the samples is the same and there is no loss of estradiol during elution step. Standard addition is used to quantify the estradiol binding in each sample.

\subsubsection{Standard addition method}

In standard addition, known concentration of estradiol is added to each sample. The amount of unknown estradiol is deduced from the increase in analytical signal. The response to increasing concentrations of added known estradiol produced a linear response. Since the sample preparation involves use of nanoparticles and multiple washing steps with high salt containing buffer, the matrix affects the analytical signal. Hence, standard addition method is more appropriate to these samples. The assumption is that the matrix effect is same on added estradiol as it has on the original estradiol concentration in the unknown. 


\subsubsection{DNA determination}

The DNA concentration in each sample is determined using Thermo Scientific Nanodrop 1000 using stacking buffer spiked with steroid as the blank. Nanodrop 1000 is a UV-vis spectrophotometer which can provide full spectrum data (200$800 \mu \mathrm{m})$. It has a compact design and uses very small volumes of samples $(0.5-$ $2.0 \mu \mathrm{L})$. It is especially used for nucleic acid or protein concentration measurements. Though the DNA concentration introduced initially in each sample is the same, the concentration varies during the sample preparation due to loss of nanoparticles in washes. So, the DNA recovered from each sample is normalized by dividing the concentration in a single set by lowest concentration of DNA within the set. The bound estradiol is then divided by this factor. Assuming the relative losses of DNA on all the samples is same, concentration is normalized based on lowest DNA concentration.

\subsubsection{Plotting data}

The specific binding curve is obtained by plotting normalized estradiol bound to DNA against free estradiol used initially. The data was fit on GraphPad Prism version 4 using one-site binding model described by the equation, $Y=\left(\left(B_{\max }{ }^{*} X\right) /\right.$ $\left.\left(K_{d}+X\right)\right)+N S^{*} X$, where $B_{\max }$ is the maximal binding and $K_{d}$ is the dissociation constant and NS is the non-specific binding. 


\subsubsection{Non-specific binding}

The non specific binding curve is done using a 37 base reverse E2 primer ( 5'GCT TCC GCG CTT CAG CGC -3') of the aptamer. The added reverse compliment couples to part of the estradiol aptamer with 76 bases. So, the aptamer is not in the right configuration and the estradiol binding to this complex is considered as non-specific binding. Non-specific binding also includes the estradiol binding to the surface of nanoparticles as well. The value of non-specific binding was estimated using slope of the curve 0.0013 .

Nonlinear regression analysis of the binding curve gave a dissociation constant of $K_{d}=11 \pm 4 \mu \mathrm{M}$ and $B_{\max }=0.43 \pm 0.03 \mu \mathrm{M}$ with an $R^{2}=0.908$. The binding curves are repeated three times to ensure the reproducibility of the results. The results are shown in the table 4-2 with $K_{d}$ and $B_{\max }$ values. The binding curves are shown in figure 4-13.

\subsection{6 "Magnetic micro particles" Vs "magnetic nanoparticles"}

Previously, the binding affinity of the 76 mer aptamer to estradiol is determined using magnetic microbeads with streptavidin surface to aid the immobilization of aptamer. ${ }^{23}$ The size of the microbeads used in our previous work is 1 um $(1000 \mathrm{~nm})$. An approximate binding capacity of 500 picomoles of single stranded 20 base pair biotinylated oligonucleotide per $\mathrm{mg}$ of bead was reported by the manufacturer. The volume of beads used was $40 \mu \mathrm{L}$ (concentration $5 \mathrm{mg} / \mathrm{ml}$ ). Thus, these beads can bind a maximum DNA concentration of $\sim 2 \mu \mathrm{M}$ (80 
picomoles). There is no shelf life for these beads. Microbeads are easy to concentrate in solution by placing them on magnet due to their larger size. The larger size of beads also tends to cause less loss of beads during sample preparation steps. The $K_{d}$ value reported with magnetic microbeads is $K_{d}=70 \pm$ $10 \mu \mathrm{M}$ and $\mathrm{a} \mathrm{B}_{\max }=0.42 \pm 0.03 \mu \mathrm{M}$.

In the present paper, superparamagnetic iron oxide nanoparticles (SPIONs) of 30 $\mathrm{nm}$ size with streptavidin surface are used. The SPIONs purchased have a shelf life of three months and noticeably they did not give good results after the expiration date. The aptamer is immobilized on streptavidin modified SPION surface and the bound and unbound estradiol separation during the sample preparation is done in a micro capillary. The smaller size of SPIONs accounts for their greater stability in solution and the time required to concentrate them on a magnet is much higher compared to microbeads. The loss of nanoparticles during the sample preparation is more due to sticking of SPIONs to glass micro capillary and the eppendrof tubes used for reconstitution of samples.

From the experimental results obtained with SPIONs, $K_{d}=11 \pm 4 \mu \mathrm{M}$ and $B_{\max }=$ $0.43 \pm 0.03 \mu \mathrm{M}$. It is shown that the binding affinity constant $K_{d}$ of the aptamer is approximately 8 times lesser than that obtained using the magnetic micro beads which tells that the binding of estradiol to aptamer immobilized on SPIONs is occurring at a higher rate. This can be explained by the higher surface area of the nanoparticles and compact packing of biotinylated aptamer on the 
streptavidin coated nanoparticles. The comparison of $\mathrm{K}_{d}$ and $\mathrm{B}_{\max }$ obtained from two studies are shown in table 4-1.

Table 4-1: Comparison of $K_{d}$ and $B_{\max }$ values obtained from the aptamer binding studies done using magnetic micro and nano particles.

\begin{tabular}{|c|c|c|c|}
\hline Capture elements & $\begin{array}{c}\text { Dissociation constant } \\
\left(K_{d}\right)\end{array}$ & $\mathbf{B}_{\max }$ & $\begin{array}{c}\text { Non-specific } \\
\text { binding slope }\end{array}$ \\
\hline SPIONs & $11 \pm 4 \mu \mathrm{M}$ & $0.43 \pm 0.03 \mu \mathrm{M}$ & 0.0013 \\
\hline microbeads & $70 \pm 10 \mu \mathrm{M}$ & $0.42 \pm 0.03 \mu \mathrm{M}$ & 0.0016 \\
\hline
\end{tabular}


Figure 4-12: Representative electropherogram showing a single standard addition determination. DNA in the sample is separated from estradiol and is represented by the peak at $1.75 \mathrm{~min}$. Inset shows the traces of bound estradiol. The known concentrations of estradiol added to the sample gave a linear response. The concentration of the unknown estradiol in the sample is then calculated.

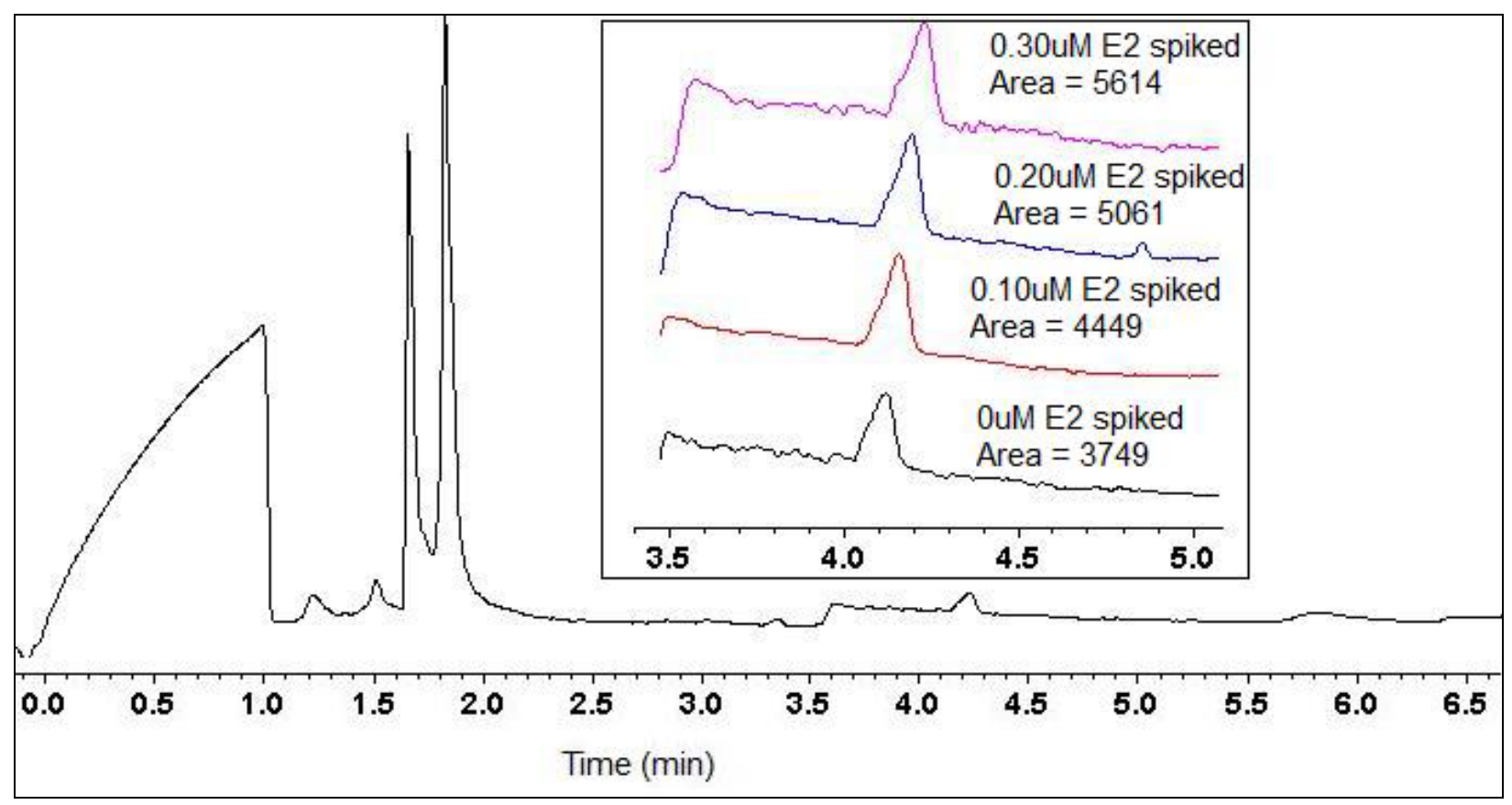


Figure 4-13: Represents three determinations of binding affinity of $76 \mathrm{mer}$ aptamer and steroids using SPIONs as capture elements. The ratio of bound estradiol to total DNA is plotted on the $y$-axis and initial free concentration of estradiol is plotted on $x$-axis. The equation used to fit non-linear data is $Y=\left(\left(B_{\max }\right.\right.$ $\left.\left.{ }^{*} \mathrm{X}\right) /\left(\mathrm{K}_{\mathrm{d}}+\mathrm{X}\right)\right)+N \mathrm{~S}^{*} \mathrm{X}$, where $\mathrm{B}_{\max }$ is the maximal binding and $\mathrm{K}_{\mathrm{d}}$ is the dissociation constant and NS is the non-specific binding.

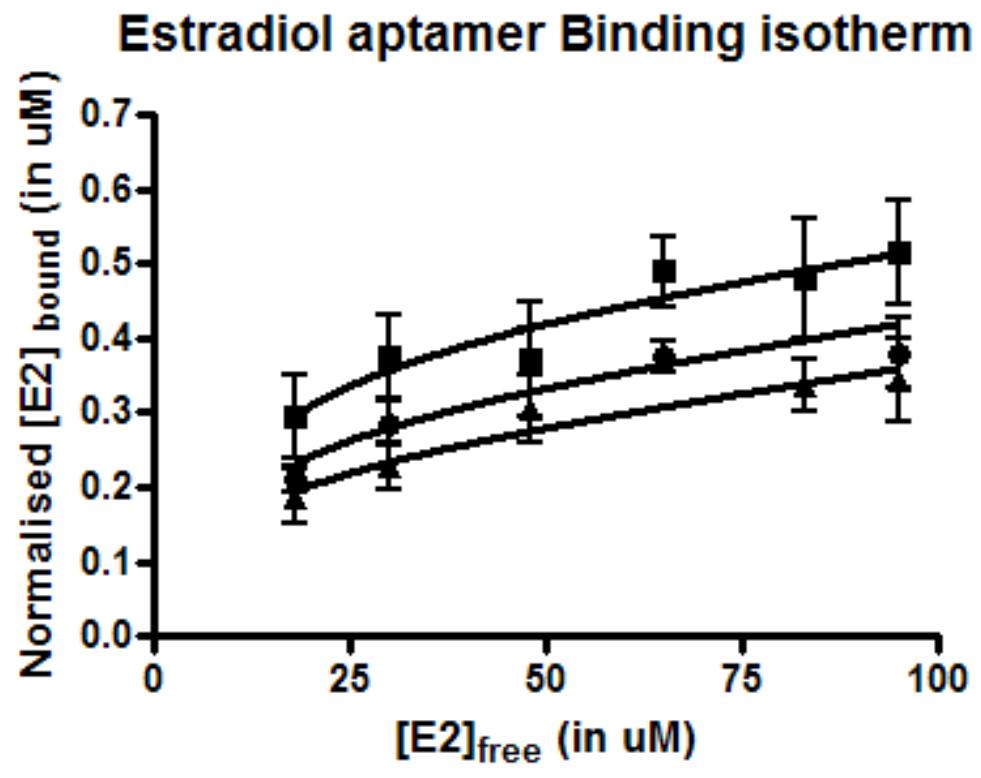


Table 4-2: Raw Data Used for each Binding Isotherm

\begin{tabular}{|c|c|c|c|c|c|}
\hline Total E2 & Total DNA & Bound E2 & uncertainty & Free E2 & $\begin{array}{c}\text { Normalized } \\
\text { Bound E2 }\end{array}$ \\
\hline \multicolumn{6}{|l|}{ Set 1} \\
\hline 18 & 0.65 & 0.36 & 0.06 & 17.64 & 0.29 \\
\hline 30 & 0.81 & 0.56 & 0.06 & 29.44 & 0.37 \\
\hline 48 & 1.18 & 0.81 & 0.08 & 47.19 & 0.37 \\
\hline 65 & 0.54 & 0.49 & 0.05 & 64.51 & 0.49 \\
\hline 83 & 0.83 & 0.74 & 0.08 & 82.26 & 0.48 \\
\hline 95 & 0.58 & 0.56 & 0.07 & 94.44 & 0.52 \\
\hline \multicolumn{6}{|c|}{$\mathrm{K}_{\mathrm{d}}=11 \pm 4$} \\
\hline \multicolumn{6}{|c|}{$B_{\max }=0.43 \pm 0.03$} \\
\hline \multicolumn{6}{|l|}{ Set 2} \\
\hline 18 & 0.57 & 0.19 & 0.04 & 17.81 & 0.19 \\
\hline 30 & 1.05 & 0.42 & 0.03 & 29.58 & 0.23 \\
\hline 48 & 0.78 & 0.42 & 0.05 & 47.58 & 0.31 \\
\hline 83 & 0.79 & 0.46 & 0.03 & 82.54 & 0.34 \\
\hline 95 & 0.95 & 0.57 & 0.06 & 94.43 & 0.34 \\
\hline \multicolumn{6}{|c|}{$K_{d}=9 \pm 4$} \\
\hline \multicolumn{6}{|c|}{$B_{\max }=0.26 \pm 0.02$} \\
\hline \multicolumn{6}{|l|}{ Set 3} \\
\hline 18 & 0.75 & 0.24 & 0.02 & 17.76 & 0.21 \\
\hline 30 & 1.41 & 0.61 & 0.03 & 29.39 & 0.29 \\
\hline 48 & 0.77 & 0.43 & 0.04 & 47.57 & 0.37 \\
\hline 65 & 0.66 & 0.38 & 0.02 & 64.62 & 0.38 \\
\hline 95 & 1.24 & 0.72 & 0.05 & 94.28 & 0.38 \\
\hline \multicolumn{6}{|c|}{$\mathrm{K}_{\mathrm{d}}=10 \pm 6$} \\
\hline$B_{\max }=C$ & $32 \pm 0.04$ & & & & \\
\hline
\end{tabular}




\subsection{Conclusions and future directions}

We have developed an effective method of capturing estradiol using aptamer anchored magnetic nanoparticles. SPIONs are proven to be effective platform for analytical sensing. Furthermore, this method can be extended to other small molecules for which aptamers have been developed. Using different aptamers, multiple compounds can be separated. This will help in detecting multiple compounds in a variety of samples using micro scale devices. SPIONs could be easier to incorporate in micro or nano scale biosensors. This work can be considered as a preliminary study to show that the magnetic nanoparticles can be more effective separation tools compared to microbeads. Further studies on using aptamers for separation of steroids in-capillary are being conducted. 


\section{References}

1. Medintz, I. L.; Uyeda, H. T.; Goldman, E. R.; Mattoussi, H., Quantum dot bioconjugates for imaging, labelling and sensing. Nature Materials 2005, 4, (6), 435-446.

2. Yan, J. L.; Estevez, M. C.; Smith, J. E.; Wang, K. M.; He, X. X.; Wang, L.; Tan, W. H., Dye-doped nanoparticles for bioanalysis. Nano Today 2007, 2, (3), 44-50.

3. Selvan, S. T.; Tan, T. T. Y.; Yi, D. K.; Jana, N. R., Functional and Multifunctional Nanoparticles for Bioimaging and Biosensing. Langmuir 2010, 26, (14), 11631-11641.

4. $\quad$ Cheng, F. Y.; Su, C. H.; Yang, Y. S.; Yeh, C. S.; Tsai, C. Y.; Wu, C. L.; Wu, M. T.; Shieh, D. B., Characterization of aqueous dispersions of Fe3O4 nanoparticles and their biomedical applications. Biomaterials 2005, 26, (7), 729738.

5. Tamer, U.; Gundogdu, Y.; Boyaci, I. H.; Pekmez, K., Synthesis of magnetic core-shell $\mathrm{Fe}(3) \mathrm{O}(4)-\mathrm{Au}$ nanoparticle for biomolecule immobilization and detection. Journal of Nanoparticle Research 2010, 12, (4), 1187-1196.

6. Yi, D. K.; Selvan, S. T.; Lee, S. S.; Papaefthymiou, G. C.; Kundaliya, D.; Ying, J. Y., Silica-coated nanocomposites of magnetic nanoparticles and quantum dots. Journal of the American Chemical Society 2005, 127, (14), 49904991. 
7. Cutler, J. I.; Zheng, D.; Xu, X. Y.; Giljohann, D. A.; Mirkin, C. A., Polyvalent Oligonucleotide Iron Oxide Nanoparticle "Click" Conjugates. Nano Letters 2010, 10, (4), 1477-1480.

8. Stoltenburg, R.; Reinemann, C.; Strehlitz, B., SELEX-A (r)evolutionary method to generate high-affinity nucleic acid ligands. Biomolecular Engineering 2007, 24, (4), 381-403.

9. Tombelli, S.; Mascini, M., Aptamers as molecular tools for bioanalytical methods. Current Opinion in Molecular Therapeutics 2009, 11, (2), 179-188.

10. Hamula, C. L. A.; Guthrie, J. W.; Zhang, H.; Li, X.-F.; Le, X. C., Selection and analytical applications of aptamers. TrAC Trends in Analytical Chemistry 2006, 25, (7), 681-691.

11. Cho, E. J.; Lee, J. W.; Ellington, A. D., Applications of Aptamers as Sensors. In Annual Review of Analytical Chemistry, Annual Reviews: Palo Alto, 2009; Vol. 2, pp 241-264.

12. Mehta, J.; Rouah-Martin, E.; Van Dorst, B.; Maes, B.; Herrebout, W.; Scippo, M.-L.; Dardenne, F.; Blust, R.; Robbens, J., Selection and Characterization of PCB-Binding DNA Aptamers. Analytical Chemistry 2012, 84, (3), 1669-1676.

13. Song, S.; Wang, L.; Li, J.; Fan, C.; Zhao, J., Aptamer-based biosensors. TrAC Trends in Analytical Chemistry 2008, 27, (2), 108-117.

14. El-Sayed, I. H.; Huang, X. H.; El-Sayed, M. A., Selective laser photothermal therapy of epithelial carcinoma using anti-EGFR antibody conjugated gold nanoparticles. Cancer Letters 2006, 239, (1), 129-135. 
15. Wolcott, A.; Gerion, D.; Visconte, M.; Sun, J.; Schwartzberg, A.; Chen, S. W.; Zhang, J. Z., Silica-coated CdTe quantum dots functionalized with thiols for bioconjugation to IgG proteins. Journal of Physical Chemistry B 2006, 110, (11), 5779-5789.

16. Andrake, M.; Guild, N.; Hsu, T.; Gold, L.; Tuerk, C.; Karam, J., DNAPolymerase of Bacteriophage-T4 Is an Autogenous Translational Repressor. Proceedings of the National Academy of Sciences of the United States of America 1988, 85, (21), 7942-7946.

17. Baker, B. R.; Lai, R. Y.; Wood, M. S.; Doctor, E. H.; Heeger, A. J.; Plaxco, K. W., An electronic, aptamer-based small-molecule sensor for the rapid, labelfree detection of cocaine in adulterated samples and biological fluids. Journal of the American Chemical Society 2006, 128, (10), 3138-3139.

18. Tombelli, S.; Minunni, A.; Mascini, A., Analytical applications of aptamers. Biosensors \& Bioelectronics 2005, 20, (12), 2424-2434.

19. Tombelli, S.; Minunni, M.; Mascini, M., Aptamers-based assays for diagnostics, environmental and food analysis. Biomolecular Engineering 2007, 24, (2), 191-200.

20. Kim, Y. S.; Jung, H. S.; Matsuura, T.; Lee, H. Y.; Kawai, T.; Gu, M. B., Electrochemical detection of 17 beta-estradiol using DNA aptamer immobilized gold electrode chip. Biosensors \& Bioelectronics 2007, 22, (11), 2525-2531.

21. Huy, G. D.; Jin, N.; Yin, B. C.; Ye, B. C., A novel separation and enrichment method of 17 beta-estradiol using aptamer-anchored microbeads. Bioprocess and Biosystems Engineering 2011, 34, (2), 189-195. 
22. Bykova, L.; Holland, L. A., Stacking enhanced determination of steroids by CE. Electrophoresis 2008, 29, (18), 3794-3800.

23. Langan, T. J.; Nyakubaya, V. T.; Casto, L. D.; Dolan, T. D.; ArcherHartmann, S. A.; Yedlapalli, S. L.; Sooter, L. J.; Holland, L. A., Assessment of aptamer-steroid binding using stacking-enhanced capillary electrophoresis. Electrophoresis 2012, 33, (5), 866-869.

24. Munro, N. J.; Palmer, J.; Stalcup, A. M.; Landers, J. P., Charged cyclodextrin-mediated sample stacking in micellar capillary electrophoresis - A simple method for enhancing the detection sensitivity of hydrophobic compounds. Journal of Chromatography B 1999, 731, (2), 369-381.

25. Schomburg, G., Problems and Achievements in the Instrumentation and Column Technology for Chromatography and Capillary Electrophoresis. Chromatographia 1990, 30, (9-10), 500-508.

26. Dose, E. V.; Guiochon, G. A., High-Resolution Modeling of Capillary Zone Electrophoresis and Isotachophoresis. Analytical Chemistry 1991, 63, (11), 10631072.

27. Dulffer, T.; Herb, R.; Herrmann, H.; Kobold, U., Capillary Electrophoresis Basic Considerations for Industrial Applications. Chromatographia 1990, 30, (1112), 675-685.

28. Kasicka, V.; Prusik, Z., Application of Capillary Isotachophoresis in Peptide Analysis. Journal of Chromatography-Biomedical Applications 1991, 569, (1-2), 123-174. 
29. Thibault, P.; Paris, C.; Pleasance, S., Analysis of Peptides and Proteins by Capillary Electrophoresis Mass-Spectrometry Using Acidic Buffers and Coated Capillaries. Rapid Communications in Mass Spectrometry 1991, 5, (10), 484-490.

30. Janini, G. M.; Issaq, H. J., Micellar Electrokinetic Capillary Chromatography - Basic Considerations and Current Trends. Journal of Liquid Chromatography 1992, 15, (6-7), 927-960.

31. Fanali, S., Enantioselective determination by capillary electrophoresis with cyclodextrins as chiral selectors. Journal of Chromatography A 2000, 875, (1-2), 89-122.

32. Nishi, H.; Fukuyama, T.; Terabe, S., Chiral Separation by CyclodextrinModified Micellar Electrokinetic Chromatography. Journal of Chromatography 1991, 553, (1-2), 503-516.

33. Kuhn, R.; Stoecklin, F.; Erni, F., Chiral Separations by Host-Guest Complexation with Cyclodextrin and Crown-Ether in Capillary Zone Electrophoresis. Chromatographia 1992, 33, (1-2), 32-36.

34. Holmberg, A.; Blomstergren, A.; Nord, O.; Lukacs, M.; Lundeberg, J.; Uhlen, M., The biotin-streptavidin interaction can be reversibly broken using water at elevated temperatures. Electrophoresis 2005, 26, (3), 501-510. 


\section{SRI LAKSHMI YEDLAPALLI}

E-mail: srilakshmi.yedlapalli@gmail.com

Mobile \#: 979-481-9133

\section{Education}

West Virginia University, Morgantown, WV

$2008-2012$

Ph.D. Analytical Chemistry

GPA - $3.89 / 4.0$

Dissertation: Magnetic nanoparticles for bio-analytical applications

Chalapathi Institute of Pharmaceutical Sciences,

$2004-2008$

Acharya Nagarjuna University, India

GPA - $3.9 / 4.0$

Bachelors in Pharmacy

\section{Relevant Course Work}

Chemical Separations, Mass Spectrometry, Physics \& Chemistry of Nanostructures, Microscopy \& Spectroscopy of Materials, Biochemical Toxicology, Biophysical chemistry, Environmental Chemistry, Crystallography, Biopharmaceutics, Biochemistry, Pharmaceutical Analysis, Pharmaceutical Microbiology, Pharmacology, Pharmaceutics, Pharmacognosy, Organic Chemistry, Medicinal Chemistry

\section{Research Experience}

\section{Graduate research experience}

- Synthesis and Surface modification of Super Paramagnetic Iron oxide nanoparticles [SPIONs] for tumor targeted drug delivery

- Assessment of Aptamer-Steroid Binding Using Stacking Enhanced Capillary Electrophoresis

- Aptamer selection against Bromacil by SELEX (Systematic Enrichment of Ligands by Exponential enrichment)

\section{Undergraduate research experience}

- Carrier mediated dissolution rate enhancement of Irbesartan

- Development of Reverse Phase High Performance Liquid Chromatographic [RP-HPLC] method for determination of Amlexanox in bulk and formulation

\section{Instruments Handling Experience}

- HPLC, GC, FTIR, UV-Vis \& Fluorescence Spectrophotometer

- DLS, TGA, XPS \& XRD

- Capillary Electrophoresis

- Disintegration and Dissolution Apparatus (8 Baskets) \& Multi station tablet compression machine

- Transmission Electron, Scanning Electron Microscopes[TEM \& SEM]

\section{Other Technical Skills}

- Significant experience in preparation and characterization of magnetic nanoparticles.

- Extensively performed Polymerase chain reactions and gel electrophoresis on nucleic acids.

- Extraordinary experience in working with multidisciplinary projects.

- Three years of graduate teaching experience in general chemistry labs for undergraduates.

- Proficient in data Analysis using Microsoft office, Image J and Igor pro software.

- Actively involved in lab scale formulation and extensive experience in in vitro dissolution testing of various pharmaceutical dosage forms such as tablets, capsules, suspensions and emulsions. 
- WVU Dissertation Fellowship at West Virginia University in Fall 2012.

- Best Poster Award at $241^{\text {st }}$ ACS National Meeting in the Division of Colloids and Surfaces at Anaheim, CA in 2011 and recognized by WV Nano.

- WVU Eberly college of Arts \& Science Graduate student travel awards in 2011 \& 2012.

- Actively mentored WV Nano SURE participants in summer 2010.

- Qualified in Graduate Aptitude Test in Engineering and Pharmaceutical Sciences (GATE) in 2007 \& 2008 with 94.3 percentile in India.

- Outstanding Undergraduate Award at Chalapathi Institute of Pharmaceutical Sciences, Acharya Nagarjuna University, India in 2008.

- Achieved many awards of merit during under graduation for academic excellence (class topper in all 4 years of B.Pharmacy).

- Best poster Award at summer undergraduate research symposium at Acharya Nagarjuna University, India in 2007.

- Won many accolades in field hockey.

Papers and Presentations

- Sri Lakshmi Yedlapalli, Aaron L. Routzan, G. M. White and R. Lloyd Carroll "Hydrodynamic size variation and stability of triblock copolymer-modified superparamagnetic iron oxide nanoparticles: Implications for drug delivery." 2012 (Manuscript in preparation).

- Sri Lakshmi Yedlapalli, Anand Narayanan, Emily C. Despeaux, Peter M. Gannett, Diandra Leslie-Pelecky, and R. Lloyd Carroll, "Antisense Survivin coupled surface modified magnetic nanoparticles for targeted cancer therapy." 2012 (Manuscript in preparation).

- Langan, T. J.; Nyakubaya, V. T.; Casto, L. D.; Dolan, T. D.; Archer-Hartmann, S. A.; Yedlapalli, S. L.; Sooter, L. J.; Holland, L. A., "Assessment of aptamer-steroid binding using stackingenhanced capillary electrophoresis". Electrophoresis 2012, 33, (5), 866-869.

- Sri Lakshmi Yedlapalli, Aaron L. Routzan, G. M. White and R. Lloyd Carroll, "Investigations on water-soluble triblock copolymer modified mono disperse superparamagnetic iron oxide nanoparticles hydrodynamic size variation and stability: Implications for drug delivery", 86th Colloid and Surface Science Symposium at Johns Hopkins University, Baltimore, MD, 2012.

- Sri Lakshmi Yedlapalli, G. M. White and R. Lloyd Carroll "Hydrodynamic size variation and stability of triblock copolymer-modified superparamagnetic iron oxide nanoparticles: Implications for drug delivery" $241^{\text {st }}$ ACS National Meeting, Colloids and Surface Chemistry Poster Session, Anaheim, CA, 2011.

- Sri Lakshmi Yedlapalli, Anand Narayanan, Peter M. Gannett and R. Lloyd Carroll "Multifunctional Superparamagnetic Iron Oxide Nanoparticles for Targeted Cancer Therapy." $241^{\text {st }}$ ACS National Meeting, Colloids and Surface Chemistry Poster Session, Anaheim, CA, 2011.

- Sri Lakshmi Yedlapalli, Ryan M. Williams, Lisa A.Holland and Letha J. Sooter "Aptamer selection against environmental pollutants by conventional and CE-SELEX" School of Pharmacy Research day, Morgantown, WV, 2011.

- Anand Narayanan, R.Lloyd Carroll, Sri Lakshmi Yedlapalli, Betty Mei and Peter M. Gannett "Multifaceted Superparamagnetic Iron Oxide Nanoparticles as Cancer Therapeutic Agents" Annual Biomedical Research Conference for Minority Students, Charlotte, NC, 2010.

- G. M. White, Sri Lakshmi Yedlapalli and R. Lloyd Carroll "Pluronic-stabilized Super Paramagnetic Iron Oxide Nanoparticles in Aqueous Solutions for Drug Delivery." WVU Nano SURE Summer Poster Session, Morgantown, WV, 2010.

- Jamie A. Barr, Anand Narayanan, Sri Lakshmi Yedlapalli, R. Lloyd Carroll, and Peter M. Gannett "Superparamagnetic Iron Oxide Nanoparticles for Treatment of Diseases." WVU Nano SURE Summer Poster Session, Morgantown, WV, 2010. 
- Sri Lakshmi Yedlapalli, M. E. Gupta, U. M. Rao and Rama Rao Nadendla "Carrier mediated dissolution rate enhancement of Irbesartan." Summer Undergraduate Research poster session, AP, India, 2008.

- Sri Lakshmi Yedlapalli and Rama Rao Nadendla "Extraction and characterization of piperine (alkaloid) from Black pepper by microwave extraction" Summer Pharmaceutical Science Exhibition, AP, India, 2007.

\section{Professional Memberships}

- American Chemical Society (ACS)

- Phi Lambda Upsilon Honorary Society (PLU) 